Supporting Information

\title{
Synthesis, Characterization and Biological Properties of Steroidal Ruthenium(II) and Iridium(III) Complexes based on the Androst- 16-en-3-ol Framework
}

Vanessa Kocha, Anna Meschkova,b, Wolfram Feuerstein ${ }^{c}$, Juliana Pfeifer ${ }^{\mathrm{b}}$, Olaf Fuhr ${ }^{\mathrm{d}}$,

Martin Nieger ${ }^{e}$, Ute Schepers ${ }^{a, b}$, Stefan Bräse ${ }^{a, f \star}$

a Institute of Organic Chemistry (IOC), Karlsruhe Institute of Technology (KIT), Fritz-Haber-Weg 6, 76131 Karlsruhe, Germany. Fax: (+49)-721-6084-8581; phone: (+49)-721-6084-2903; e-mail: braese@kit.edu.

b Institute of Functional Interfaces (IFG), Karlsruhe Institute of Technology (KIT), Hermann von Helmholtz Platz 1, 76344 Eggenstein-Leopoldshafen, Germany.

C Institute of Inorganic Chemistry, Division Molecular Chemistry, Karlsruhe Institute of Technology (KIT), Engesserstr. 15, 76131 Karlsruhe (Germany).

d Institute for Nanotechnology (INT) and Karlsruhe Nano Micro Facility (KNMF), Karlsruhe Institute of Technology (KIT), Hermann von Helmholtz Platz 1, 76344 Eggenstein-Leopoldshafen, Germany.

e Department of Chemistry, University of Helsinki, P. O. Box 55, 00014 University of Helsinki, Finland.

f Institute of Toxicology and Genetics, Karlsruhe Institute of Technology (KIT), Hermann-von-Helmholtz-Platz 1, D-76344 Eggenstein-Leopoldshafen, Germany. 



\section{Contents}

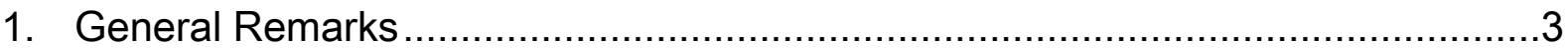

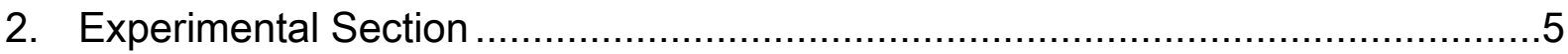

2.1. Synthetic procedures for the steroidal ligands .....................................

2.2. Synthetic procedures for the ruthenium(II) and iridium(III) complexes .......... 10

2.3. NMR spectra of the synthesized compounds ....................................... 18

2.4 Additional 2D NMR spectra for selected compounds ................................32

2.5. X-ray crystallography .......................................................... 43

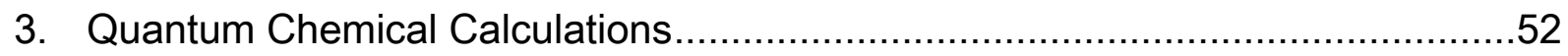

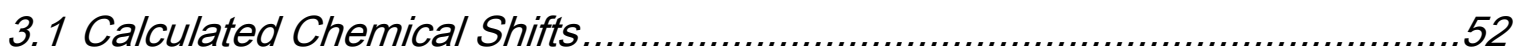

3.2 Computational Studies........................................................... 54

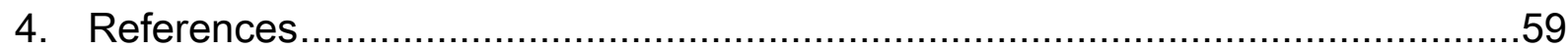




\section{General Remarks}

NMR spectra were recorded on a Bruker Avance 400, Bruker Avance III HD, or Bruker Avance 600 spectrometer as solutions at room temperature. Chemical shifts $\delta$ are expressed in parts per million (ppm) downfield from tetramethylsilane (TMS). References for ${ }^{1} \mathrm{H} N M R$ and ${ }^{13} \mathrm{C}$ NMR were the residual solvent peaks of $d_{1}$-chloroform $\left({ }^{1} \mathrm{H}: \delta=7.26 \mathrm{ppm} ;{ }^{13} \mathrm{C}: \delta=77.0 \mathrm{ppm}\right)$. All coupling constants $(J)$ are absolute values and are given in Hertz $(\mathrm{Hz})$. The description of signals includes: $\mathrm{s}=$ singlet, $\mathrm{d}=$ doublet, $\mathrm{t}=$ triplet, $\mathrm{q}=$ quartet, $\mathrm{m}=$ multiplet, $\mathrm{dd}=$ doublet of doublets and $\mathrm{ddd}=$ double doublet of doublets and so forth. The spectra were analyzed according to first order. The assignments of the signal structure in ${ }^{1} \mathrm{H}$ NMR were made by the multiplicity and for ${ }^{13} \mathrm{C}$ NMR by DEPT 90 - and DEPT 135 -spectra (DEPT $=$ distortionless enhancement by polarization transfer) and are described as follows: $+=$ primary or tertiary C-atom (positive DEPT-signal), $-=$ secondary $\mathrm{C}$-atom (negative signal) and $C_{\mathrm{q}}=$ quaternary C-atom (no DEPT-signal). Furthermore, 2D NMR experiments such as COSY (COSY = correlation spectroscopy) HSQC (HSQC = heteronuclear single-quantum correlation), HMBC (HMBC = heteronuclear multiple-bond correlation) or NOESY (NOESY = nuclear Overhauser effect spectroscopy) were performed in order to uniquely assign all $\mathrm{H}$ and $\mathrm{C}$ atoms. The sum of $\mathrm{H}$-atoms does not add up to the number of $\mathrm{H}$ atoms in the sum formula for the presented steroids, since the proton of the $\mathrm{OH}$ is in most cases not visible in the ${ }^{1} \mathrm{H}$ NMR spectrum.

IR spectra were recorded on a Bruker alpha-p and a FT-IR IFS 88 spectrometer. The compounds were measured as pure substances by ATR technique (ATR = attenuated total reflection). The position of the absorption band is given in wave numbers $\tilde{v}$ in $\mathrm{cm}^{-1}$ between $3600 \mathrm{~cm}^{-1}$ and $500 \mathrm{~cm}^{-1}$. The intensities of the bands were characterized as follows: vs = very strong $(0-20 \% \mathrm{~T}), \mathrm{s}=$ strong $(21-40 \% \mathrm{~T}), \mathrm{m}=$ medium $(41-60 \% \mathrm{~T}), \mathrm{w}=$ weak $(61-80 \% \mathrm{~T}), \mathrm{vw}=\operatorname{very}$ weak $(81-100 \% \mathrm{~T})$.

Mass spectra were measured by EI-MS (electron impact mass spectrometry) or FAB-MS (fast atom bombardement) and were recorded on a Finnigan MAT 95. The peaks are given as mass-to-charge-ratio $(\mathrm{m} / \mathrm{z})$. The molecule peak is given as $[\mathrm{M}]^{+}$and characteristic fragment peaks are given as [M-fragment $]^{+}$ or [fragment $]^{+}$. The signal intensities are given in percent, relatively to the intensity of the base signal $(100 \%)$. For the high resolution mass, the following abbreviations were used: calc. $=$ calculated data, found $=$ measured data.

Analytical thin layer chromatography (TLC) was carried out on Merck silica gel coated aluminum plates (silica gel $60, \mathrm{~F}_{254}$ ), detected under UV-light at $254 \mathrm{~nm}$ or stained with "Seebach staining solution" (mixture of phosphomolybdic acid, cerium(IV)-sulfate tetrahydrate, sulfuric acid and water).

Solvent mixtures are understood as volume/volume. Solvents, reagents and chemicals were purchased from Aldrich, Fluka, Carbolution, ChemPur, ABCR, TCI and Fisher Scientific. All solvents, reagents and chemicals were used as purchased unless stated otherwise. Solvents were removed at $40{ }^{\circ} \mathrm{C}$ at the rotavapor.

Dichloromethane (DCM) was destilled from $\mathrm{CaH}_{2}$ under argon atmosphere and tetrahydrofuran (THF) was destilled from $\mathrm{Na} /$ benzophenone under argon atmosphere before prior use. Methanol $(\mathrm{MeOH}$, 
99.9\%) and $N, N$-Dimethylformamid (DMF, 99.8\%) were purchased as acroseal bottles from ACROS Organics $^{\mathrm{TM}}$.

Air- or moisture-sensitive reactions were carried out under argon atmosphere in oven-dried and previously evacuated and heated glass ware. Liquids were transferred with plastic syringes and steel cannula. Solids were used as powder.

Reaction control was performed by thin layer chromatography. If not stated otherwise, crude products were purified by flash chromatography on silica gel by the procedure of Still. ${ }^{1}$ Silica gel $60(0.040 \times$ $0.063 \mathrm{~mm}$, Geduran ${ }^{\circledR}$, Merck) was used as stationary phase and solvents of p.a. quality as mobile phase were used. 


\section{Experimental Section}

\subsection{Synthetic procedures for the steroidal ligands}

Please note that the protocols for the synthesis of the steroidal ligands are already published in $A d v$. Synth. Catal. 2017, 359, 832-840.2

\section{$\underline{(3 \beta, 5 \alpha)-17-B r o m o a n d r o s t-16-e n-3-o l ~(4 a))^{2}}$}

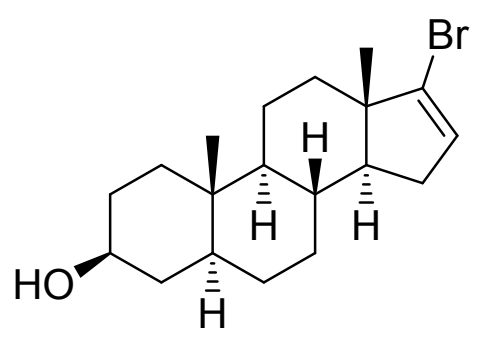

The synthesis of $(3 \beta, 5 \alpha)$-17-bromoandrost-16-en-3-ol (4a) followed a modified, reported procedure. $^{3}$ A solution of epi-androsterone 3 (500 mg, $1.72 \mathrm{mmol}, 1.00$ equiv.), hydrazine monohydrate $(1.10 \mathrm{~mL}$, 34.4 mmol, 20.0 equiv.) and $\mathrm{NEt}_{3}(4.80 \mathrm{~mL}, 34.4 \mathrm{mmol}, 20.0$ equiv.) in $\mathrm{EtOH}(25 \mathrm{~mL})$ was heated under argon atmosphere for $16 \mathrm{~h}$ to $50^{\circ} \mathrm{C}$. After cooling, the solvents were removed and the obtained colorless powder was dissolved in dry pyridine $(7.0 \mathrm{~mL})$ under argon atmosphere. NBS (918 $\mathrm{mg}, 5.16 \mathrm{mmol}, 3.00$ equiv.) in dry pyridine $\left(7.0 \mathrm{~mL}\right.$ ) was added slowly at $0{ }^{\circ} \mathrm{C}$. The mixture was stirred for $1 \mathrm{~h}$ at room temperature and afterwards quenched with $1 \mathrm{M}$ aqueous $\mathrm{HCl}$ solution. The product was extracted with ethyl acetate $(3 \times 50 \mathrm{~mL})$ and the combined organic layers were washed with saturated aqueous $\mathrm{NaHCO}_{3}(40 \mathrm{~mL})$ and brine $(40 \mathrm{~mL})$. The organic phase was dried over $\mathrm{Na}_{2} \mathrm{SO}_{4}$ and the solvent was removed under reduced pressure. The residue was purified by flash column chromatography on silica gel (cyclohexane/ethyl acetate, $5: 1)$ to afford the title compound $\mathbf{4 a}(461 \mathrm{mg}, 1.30 \mathrm{mmol}, 76 \%)$ as a colorless powder.

$\boldsymbol{R}_{f}=0.22(c \mathrm{Hex} / \mathrm{EtOAc}, 3: 1) .{ }^{1} \mathbf{H}$ NMR $\left(400 \mathrm{MHz}, \mathrm{CDCl}_{3}\right): \delta=5.81(\mathrm{dd}, J=3.3,1.7 \mathrm{~Hz}, 1 \mathrm{H},=\mathrm{CH})$, $3.59(\mathrm{tt}, J=15.9,11.1,4.8 \mathrm{~Hz}, 1 \mathrm{H}, \mathrm{CHOH}), 2.11\left(\mathrm{ddd}, J=14.8,6.2,3.3 \mathrm{~Hz}, 1 \mathrm{H},=\mathrm{CHCH}_{2}{ }^{\mathrm{b}}\right), 1.87(\mathrm{ddd}$, $\left.J=14.8 .11 .1,1.8 \mathrm{~Hz}, 1 \mathrm{H},=\mathrm{CHCH}_{2}^{\mathrm{a}}\right), 1.83-1.77\left(\mathrm{~m}, 1 \mathrm{H}, \mathrm{CH}_{2}\right), 1.73-1.21(\mathrm{~m}, 12 \mathrm{H}, 8-\mathrm{CH}+7$ different $\left.\mathrm{CH}_{2}\right), 1.18-1.06(\mathrm{~m}, 1 \mathrm{H}, 5-\mathrm{CH}), 1.02-0.88\left(\mathrm{~m}, 2 \mathrm{H}, 2\right.$ different $\left.\mathrm{CH}_{2}\right), 0.83\left(\mathrm{~s}, 3 \mathrm{H}, \mathrm{CH}_{3}\right), 0.82(\mathrm{~s}, 3 \mathrm{H}$, $\left.\mathrm{CH}_{3}\right), 0.72$ (ddd, $\left.J=12.3,10.0,4.6 \mathrm{~Hz}, 1 \mathrm{H}, 9-\mathrm{CH}\right)$ ppm. $-{ }^{13} \mathbf{C}$ NMR $\left(101 \mathrm{MHz}, \mathrm{CDCl}_{3}\right): \delta=135.6$ $(=\mathrm{CH}), 128.8\left(17-\mathrm{C}_{\mathrm{q}} \mathrm{Br}\right), 71.1(+, \mathrm{CHOH}), 55.3(+, \mathrm{CH}), 54.5(+, \mathrm{CH}), 48.6\left(13-C_{\mathrm{q}}\right), 44.9(+, 5-\mathrm{CH})$, $38.0\left(-, \mathrm{CH}_{2}\right), 36.6\left(-, \mathrm{CH}_{2}\right), 35.5\left(10-\mathrm{C}_{\mathrm{q}}\right), 34.4\left(-, \mathrm{CH}_{2}\right), 34.1(+, 8-\mathrm{CH}), 31.6\left(-, \mathrm{CH}_{2}\right), 31.3$ $\left(-, \mathrm{CH}_{2}\right), 31.2\left(-, \mathrm{CH}_{2}\right), 28.3\left(-, 6-\mathrm{CH}_{2}\right), 21.1\left(-, 11-\mathrm{CH}_{2}\right), 15.1\left(+, 18-\mathrm{CH}_{3}\right), 12.1\left(+, 19-\mathrm{CH}_{3}\right) \mathrm{ppm} .-$ IR (ATR): v = 3271 (br), 2921 (w), 2841 (w), 1588 (vw), 1450 (w), 1368 (w), 1245 (vw), 1178 (vw), 1134 (vw), 1079 (vw), 1060 (w) 1040 (w), 996 (w), 949 (vw), 919 (vw), 873 (vw), 851 (vw), 834 (vw), $820(\mathrm{vw}), 802(\mathrm{w}), 740(\mathrm{vw}), 708(\mathrm{vw}), 656$ (w), 609 (vw), $587(\mathrm{vw}), 565$ (vw), 520 (vw), 495 (vw) $\mathrm{cm}^{-1}$. - MS (EI, $\left.70 \mathrm{eV}, 100^{\circ} \mathrm{C}\right): \mathrm{m} / z(\%)=354(17)+352(17)[\mathrm{M}]^{\circ+}, 339(38)+337(40)$ $\left[\mathrm{M}-\mathrm{CH}_{3}\right]^{+}, 321(17)+319(18), 273(100)[\mathrm{M}-\mathrm{Br}]^{+}, 255$ (23), 239 (28). - HRMS (EI, $\left.\mathrm{C}_{19} \mathrm{H}_{29} \mathrm{O}^{79} \mathrm{Br}\right)$ : calc. $=352.1396$; found $=352.1398$. 
<smiles>C[C@]12CC[C@H]3[C@@H](CC[C@@H]4C[C@H](O)CC[C@]34C)[C@@H]1CC=C2I</smiles>

A solution of epi-androsterone 3 (1.00 g, $3.44 \mathrm{mmol}, 1.00$ equiv.), hydrazine monohydrate $\left(2.1 \mathrm{~mL}, 68.9 \mathrm{mmol}, 20.0\right.$ equiv.) and $\mathrm{NEt}_{3}$ (9.6 mL, $68.9 \mathrm{mmol}, 20.0$ equiv.) in EtOH $(50 \mathrm{~mL})$ was heated under argon atmosphere for $18 \mathrm{~h}$ to $50^{\circ} \mathrm{C}$. After cooling, the solvents were removed and the obtained colorless powder was dissolved in dry THF $(60 \mathrm{~mL})$ and dry $\mathrm{NEt}_{3}(10 \mathrm{~mL})$ under argon atmosphere. At $0{ }^{\circ} \mathrm{C}, \mathrm{I}_{2}(1.75 \mathrm{~g}, 6.89 \mathrm{mmol} .2 .00$ equiv. $)$ in dry THF $(10 \mathrm{~mL})$ was added slowly via cannulation. The mixture was stirred for $1 \mathrm{~h}$ at room temperature and afterwards quenched with saturated aqueous solutions of $\mathrm{Na}_{2} \mathrm{~S}_{2} \mathrm{O}_{3}(30 \mathrm{~mL})$ and $\mathrm{NaHCO}_{3}(50 \mathrm{~mL})$. The product was extracted with ethyl acetate $(3 \times 100 \mathrm{~mL})$ and the combined organic layers were washed with brine $(40 \mathrm{~mL})$. The organic phase was dried over $\mathrm{Na}_{2} \mathrm{SO}_{4}$ and the solvent was evaporated. The residue was purified by flash chromatography on silica (cyclohexane/ethyl acetate, 5:1) to afford the title compound $\mathbf{4 b}(1.25,3.13 \mathrm{mmol}, 91 \%)$ as a colorless, light sensitive powder.

$\boldsymbol{R}_{f}=0.67(c \mathrm{Hex} /$ EtOAc, $1: 1) .{ }^{1} \mathbf{H}$ NMR $\left(500 \mathrm{MHz}, \mathrm{CDCl}_{3}\right): \delta=6.10(\mathrm{dd}, J=3.4,1.7 \mathrm{~Hz}, 1 \mathrm{H},=\mathrm{CH})$, 3.59 (tt, $J=10.4,4.6 \mathrm{~Hz}, 1 \mathrm{H}, \mathrm{CHOH}), 2.11\left(\mathrm{ddd}, J=15.0,6.4,3.2 \mathrm{~Hz}, 1 \mathrm{H},=\mathrm{CHCH}_{2}{ }^{\mathrm{a}}\right.$ ), 1.91 (ddd, $\left.J=15.0,11.0,1.7 \mathrm{~Hz}, 1 \mathrm{H},=\mathrm{CHCH}_{2}{ }^{\mathrm{b}}\right), 1.85-1.78\left(\mathrm{~m}, 1 \mathrm{H}, \mathrm{CH}_{2}\right), 1.74-1.54\left(\mathrm{~m}, 6 \mathrm{H}, 5\right.$ different $\mathrm{CH}_{2}+8-$ $\mathrm{CH}), 1.49-1.24\left(\mathrm{~m}, 6 \mathrm{H}, 4\right.$ different $\left.\mathrm{CH}_{2}+\mathrm{CH}\right), 1.21-1.08\left(\mathrm{~m}, 2 \mathrm{H}, \mathrm{CH}_{2}+5-\mathrm{CH}\right), 1.06-0.91(\mathrm{~m}, 2 \mathrm{H}, 2$ different $\left.\mathrm{CH}_{2}\right), 0.82\left(\mathrm{~s}, 3 \mathrm{H}, \mathrm{CH}_{3}\right), 0.71\left(\mathrm{~s}, 3 \mathrm{H}, \mathrm{CH}_{3}\right), 0.74-0.68(\mathrm{~m}, 1 \mathrm{H}, \mathrm{CH}) \mathrm{ppm} .-{ }^{13} \mathbf{C} \mathbf{N M R}$ $\left(101 \mathrm{MHz}, \mathrm{CDCl}_{3}\right): \delta=137.3(+,=C \mathrm{H}), 112.7\left(C_{\mathrm{q}} \mathrm{I}\right), 71.1(+, \mathrm{CHOH}), 54.6(+, \mathrm{CH}), 54.5(+, C \mathrm{H}), 49.9$ $\left(13-C_{\mathrm{q}}\right), 44.8(+, 5-\mathrm{CH}), 37.9\left(-, \mathrm{CH}_{2}\right), 36.6\left(-, \mathrm{CH}_{2}\right), 36.1\left(-, \mathrm{CH}_{2}\right), 35.5\left(10-C_{\mathrm{q}}\right), 34.40(+, 8-\mathrm{CH}), 33.5$ $\left(-, \mathrm{CH}_{2}\right), 31.4\left(-, \mathrm{CH}_{2}\right), 31.3\left(-, \mathrm{CH}_{2}\right), 28.4\left(-, 6-\mathrm{CH}_{2}\right), 21.5\left(-, 11-\mathrm{CH}_{2}\right), 15.1\left(+, 18-\mathrm{CH}_{3}\right), 12.1(+, 19-$ $\mathrm{CH}_{3}$ ) ppm. - IR (ATR): v = 3306 (br), 2921 (w), 2846 (w), 1574 (vw), 1447 (w), 1369 (w), 1244 (vw), 1231 (vw), 1133 (vw), 1079 (vw), 1039 (w), 988 (w), 949 (vw), 917 (vw), 871 (vw), 850 (vw), 834 (vw), $811(\mathrm{w}), 735(\mathrm{vw}), 705(\mathrm{vw}), 656(\mathrm{w}), 609(\mathrm{vw}), 582(\mathrm{vw}), 562(\mathrm{vw}), 495(\mathrm{vw}) \mathrm{cm}^{-1}$. - MS (EI, $\left.70 \mathrm{eV}, 100{ }^{\circ} \mathrm{C}\right): m / z(\%)=400(93)[\mathrm{M}]^{++}, 385(88)\left[\mathrm{M}-\mathrm{CH}_{3}\right]^{+}, 255$ (26), 161 (27), 148 (35), 107 (26). - HRMS (EI, $\mathrm{C}_{19} \mathrm{H}_{29} \mathrm{O}_{1}{ }^{127} \mathrm{I}_{1}$ ): calc. $=400.1258$; found $=400.1259$. 


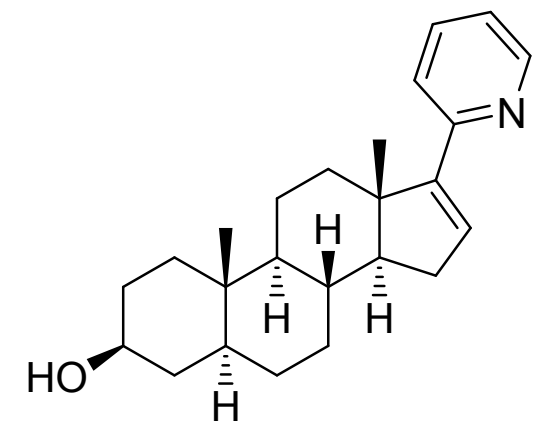

In a Schlenk tube, alkenyl iodide 4a $(150 \mathrm{mg}, 375 \mu \mathrm{mol}$, 1.00 equiv.), 2-tributylstannylpyridine ${ }^{2,} 4$ (420 mg, $1.14 \mathrm{mmol}$, 3.04 equiv.), $\mathrm{LiCl}$ (53 mg, $1.25 \mathrm{mmol}, 0.30$ equiv.) and $\mathrm{CuCl}$ (186 mg, $1.88 \mathrm{mmol}, 5.00$ equiv.) were dissolved in dry DMF $(6.0 \mathrm{~mL})$ under argon atmosphere. The reaction mixture was degassed using three freeze-pump-thaw cycles. Then, $\mathrm{Pd}\left(\mathrm{PPh}_{3}\right)_{4}$ (130 mg, $113 \mu \mathrm{mol}, 30 \mathrm{~mol} \%$ ) was added and the reaction mixture was stirred at $80^{\circ} \mathrm{C}$ for $60 \mathrm{~h}$. After cooling to room temperature. an aqueous solution of $\mathrm{KF}$ ( $3 \mathrm{M}$, 4.00 equiv.) was added and stirred for $30 \mathrm{~min}$, followed by filtration over Celite ${ }^{\circledR}$ (dichloromethane as eluent). The organic phase was washed with aqueous saturated $\mathrm{NH}_{4} \mathrm{Cl}$ solution $(100 \mathrm{~mL})$ and extracted with dichloromethane $(2 \times 100 \mathrm{~mL})$. The combined organic layers were washed with brine $(100 \mathrm{~mL})$, dried over $\mathrm{Na}_{2} \mathrm{SO}_{4}$ and filtered. The solvent was removed under reduced pressure and the title compound 5a was obtained as a colorless solid ( $98 \mathrm{mg}, 278 \mu \mathrm{mol}, 74 \%$ ) after flash column chromatography on silica gel (cyclohexane/ethyl acetate, 4:1). For the biological tests the compound was washed multiple times with $n$-hexane.

$\boldsymbol{R}_{\mathbf{f}}=0.32(c \mathrm{Hex} / \mathrm{EtOAc}, 2: 1) .{ }^{1} \mathbf{H}$ NMR $\left(400 \mathrm{MHz}, \mathrm{CDCl}_{3}\right): \delta=8.55(\mathrm{ddd}, J=4.9,1.9,1.0 \mathrm{~Hz}, 1 \mathrm{H}$, $\left.\mathrm{NCH}_{\mathrm{Ar}}\right), 7.58\left(\mathrm{td}, J=7.7,1.9 \mathrm{~Hz}, 1 \mathrm{H}, \mathrm{C} H_{\mathrm{Ar}}\right), 7.37\left(\mathrm{dt}, J=8.1,1.1 \mathrm{~Hz}, 1 \mathrm{H}, \mathrm{C} H_{\mathrm{Ar}}\right), 7.09$ (ddd, $J=7.5$, $\left.4.9,1.2 \mathrm{~Hz}, 1 \mathrm{H}, \mathrm{C} H_{\mathrm{Ar}}\right), 6.39\left(\mathrm{dd}, J=3.5,1.9 \mathrm{~Hz}, 1 \mathrm{H}, \mathrm{C}_{\mathrm{q}}=\mathrm{CH}\right), 3.60(\mathrm{tt}, J=11.0,4.7 \mathrm{~Hz}, 1 \mathrm{H}, \mathrm{CHOH})$, 2.39 (ddd, $\left.J=12.1,3.8,2.2 \mathrm{~Hz}, 1 \mathrm{H}, \mathrm{CH}_{2}\right), 2.24$ (ddd, $J=16.1,6.4,3.4 \mathrm{~Hz}, 1 \mathrm{H}, \mathrm{CH}=\mathrm{CH}_{2}$ a), 2.04 (ddd, $\left.J=16.1,11.4,1.9 \mathrm{~Hz}, 1 \mathrm{H}, \mathrm{CH}=\mathrm{CH}_{2}{ }^{\mathrm{b}}\right), 1.85-1.79\left(\mathrm{~m}, 1 \mathrm{H}, \mathrm{CH}_{2}\right), 1.76-1.46\left(\mathrm{~m}, 8 \mathrm{H}, 4\right.$ different $\mathrm{CH}_{2}+$ 8-CH+ $\mathrm{CH}), 1.40-1.23\left(\mathrm{~m}, 5 \mathrm{H}, 4\right.$ different $\left.\mathrm{CH}_{2}\right), 1.21-1.10(\mathrm{~m}, 1 \mathrm{H}, 5-\mathrm{CH}), 1.11\left(\mathrm{~s}, 3 \mathrm{H}, \mathrm{CH}_{3}\right), 1.06-$ $0.96\left(\mathrm{~m}, 2 \mathrm{H}, 2\right.$ different $\left.\mathrm{CH}_{2}\right), 0.87\left(\mathrm{~s}, 3 \mathrm{H}, \mathrm{CH}_{3}\right), 0.83-0.69(\mathrm{~m}, 1 \mathrm{H}, \mathrm{CH}) \mathrm{ppm} .-{ }^{13} \mathbf{C} \mathbf{M R}(101 \mathrm{MHz}$, $\left.\mathrm{CDCl}_{3}\right): \delta=155.5\left(C_{\mathrm{q}}\right), 154.1\left(C_{\mathrm{q}}\right), 148.8\left(6^{\prime}-C \mathrm{HN}\right), 135.7\left(+, 4^{\prime}-\mathrm{CH}_{\mathrm{Ar}}\right), 131.5\left(C_{\mathrm{q}}=\mathrm{CH}\right), 121.1\left(+, 3^{\prime}-\right.$ or $\left.4^{\prime}-\mathrm{CH}_{\mathrm{Ar}}\right), 120.7\left(+, 3^{\prime}\right.$ or $\left.4^{\prime}-\mathrm{CH}_{\mathrm{Ar}}\right), 71.2(+, \mathrm{CHOH}), 57.2(+, \mathrm{CH}), 54.6(+, \mathrm{CH}), 47.0\left(13-C_{\mathrm{q}}\right), 44.9$ $(+, 5-\mathrm{CH}), 38.1\left(-, \mathrm{CH}_{2}\right), 36.7\left(-, \mathrm{CH}_{2}\right), 35.5\left(10-\mathrm{C}_{\mathrm{q}}\right), 35.1\left(-, \mathrm{CH}_{2}\right), 33.8(+, 8-\mathrm{CH}), 31.8\left(-, \mathrm{CH}_{2}\right), 31.5$ $\left(-, \mathrm{CH}_{2}\right), 31.4\left(-, \mathrm{CH}_{2}\right), 28.5\left(-, 6-\mathrm{CH}_{2}\right), 21.0\left(-, 11-\mathrm{CH}_{2}\right), 16.2\left(+, 18-\mathrm{CH}_{3}\right), 12.2\left(+, 19-\mathrm{CH}_{3}\right) \mathrm{ppm} .-$ IR (ATR): v = 3282 (br), 2913 (w), 2848 (w), 1584 (vw), 1462 (w), 1448 (w), 1366 (vw), 1148 (vw), $1036(\mathrm{w}), 1006(\mathrm{vw}), 957(\mathrm{vw}), 923(\mathrm{vw}), 772(\mathrm{w}), 739(\mathrm{vw}), 692(\mathrm{w}), 604(\mathrm{vw}), 487(\mathrm{vw})$, $403(\mathrm{vw}) \mathrm{cm}^{-1}$. - MS (EI, $\left.70 \mathrm{eV}, 70{ }^{\circ} \mathrm{C}\right): \mathrm{m} / z(\%)=351(100)[\mathrm{M}]^{\circ+}, 336(82)\left[\mathrm{M}-\mathrm{CH}_{3}\right]^{+}, 269$ (52). HRMS (EI, $\left.\mathrm{C}_{24} \mathrm{H}_{33} \mathrm{ON}\right)$ : calc. $=351.2557$; found $=351.2555$. 


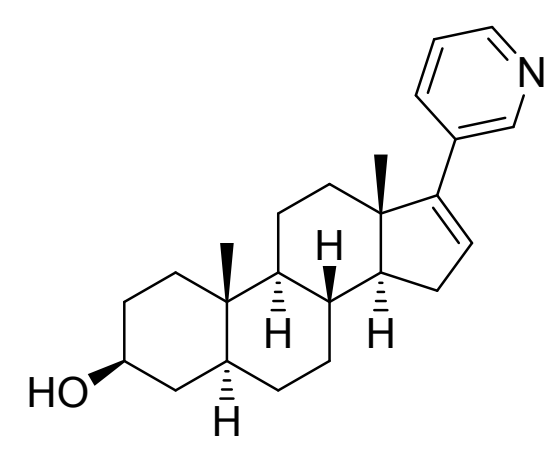

In a Schlenk tube, vinyl iodide 1-I (50 mg, $125 \mu \mathrm{mol}, 1.00$ equiv.), crude 3-tributylstannylpyridine ${ }^{2,} \quad 4 \quad(140 \mathrm{mg}, \quad 375 \mu \mathrm{mol}$, 3.00 equiv.), $\mathrm{LiCl}$ (53 mg, $1.25 \mathrm{mmol}, 10.0$ equiv.) and $\mathrm{CuCl}$ (124 mg, $1.25 \mathrm{mmol}, 10.0$ equiv.) were dissolved in dry DMF $(3.0 \mathrm{~mL})$ under argon atmosphere. The reaction mixture was degassed using three freeze-pump-thaw cycles. Then $\mathrm{Pd}\left(\mathrm{PPh}_{3}\right)_{4}$ ( $29 \mathrm{mg}, 25 \mu \mathrm{mol}, 20 \mathrm{~mol} \%$ ) was added and the reaction mixture was stirred at $80^{\circ} \mathrm{C}$ for $60 \mathrm{~h}$. After cooling to room temperature an aqueous solution of $\mathrm{KF}(3 \mathrm{M}, 4.00$ equiv.) was added and stirred for $30 \mathrm{~min}$, followed by filtration over Celite ${ }^{\circledR}$ (dichloromethane as eluent). The organic phase was washed with aqueous saturated $\mathrm{NH}_{4} \mathrm{Cl}$ solution $(50 \mathrm{~mL})$ and extracted with dichloromethane $(2 \times 50 \mathrm{~mL})$. The combined organic layers were washed with brine $(100 \mathrm{~mL})$, dried over $\mathrm{Na}_{2} \mathrm{SO}_{4}$ and filtered. The solvent was removed under reduced pressure and, after flash column chromatography on silica gel (cyclohexane/ethyl acetate, $4: 1$ ), the title compound $\mathbf{5 b}$ was obtained as a colorless solid (27 mg, $75.7 \mu \mathrm{mol}, 60 \%$ ). For the biological tests the compound was washed multiple times with $n$-hexane.

$\boldsymbol{R}_{\boldsymbol{f}}=0.18(\mathrm{cHex} / \mathrm{EtOAc}, 3: 2) .-{ }^{1} \mathbf{H}$ NMR $\left(400 \mathrm{MHz}, \mathrm{CDCl}_{3}\right): \delta=8.59\left(\mathrm{~s}, 1 \mathrm{H}, 2^{\prime}-\mathrm{CH}_{\mathrm{Pyr}}\right), 8.43(\mathrm{~d}$, $\left.J=3.2 \mathrm{~Hz}, 1 \mathrm{H}, 4^{\prime}-\mathrm{C} H_{\mathrm{Pyr}}\right), 7.63\left(\mathrm{dt}, J=8.0,2.0 \mathrm{~Hz}, 1 \mathrm{H}, 6{ }^{\prime}-\mathrm{CH}_{\mathrm{Ar}}\right), 7.20(\mathrm{dd}, J=7.8,4.8 \mathrm{~Hz}, 1 \mathrm{H}$, $\left.5^{\prime}-\mathrm{CH}_{\mathrm{Ar}}\right), 5.96\left(\mathrm{dd}, J=3.3 \mathrm{~Hz}, 1.8 \mathrm{~Hz}, 1 \mathrm{H}, \mathrm{C}_{\mathrm{q}}=\mathrm{CH}\right), 3.60(\mathrm{tt}, J=15.9,11.0,4.8 \mathrm{~Hz}, 1 \mathrm{H}, \mathrm{CHOH}), 2.22$ (ddd, $\left.J=15.7,6.4,3.3 \mathrm{~Hz}, 1 \mathrm{H},=\mathrm{CHCH}_{2}{ }^{\mathrm{a}}\right), 2.05-1.97\left(\mathrm{~m}, 2 \mathrm{H},=\mathrm{CHCH}_{2}{ }^{\mathrm{b}}+\mathrm{CH}_{2}\right), 1.82-1.79(\mathrm{~m}, 1 \mathrm{H}$, $\left.\mathrm{CH}_{2}\right), 1.82-1.51\left(\mathrm{~m}, 6 \mathrm{H}, 4\right.$ different $\left.\mathrm{CH}_{2}+8-\mathrm{CH}+\mathrm{CH}\right), 1.45-1.24\left(\mathrm{~m}, 6 \mathrm{H}, 5\right.$ different $\left.\mathrm{CH}_{2}\right), 1.17-1.10$ (m, 1H, 5-CH), 1.05-0.89 (m, 2H, 2 different $\left.\mathrm{CH}_{2}\right), 0.99$ (s, 3H, $\left.\mathrm{CH}_{3}\right), 0.85\left(\mathrm{~s}, 3 \mathrm{H}, \mathrm{CH}_{3}\right), 0.78-0.72$ (m, $1 \mathrm{H}, \mathrm{CH})$ ppm. $-{ }^{13} \mathrm{C}$ NMR $\left(101 \mathrm{MHz}, \mathrm{CDCl}_{3}\right): \delta=151.6\left(C_{\mathrm{q}}=\mathrm{CH}_{\mathrm{Ar}}\right), 147.6\left(+, 2^{\prime}-\mathrm{CH}_{\mathrm{Pyr}}\right.$ or $\left.4^{\prime}-\mathrm{CH}_{\mathrm{Pyr}}\right)$,

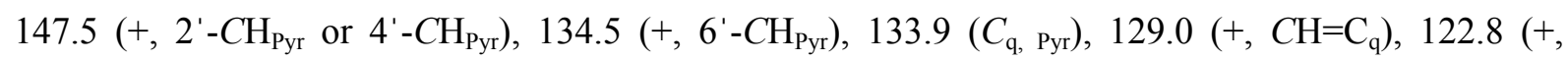
$\left.5^{\prime}-\mathrm{CH}_{\mathrm{Pyr}}\right), 71.0(+, 3-\mathrm{CHOH}), 57.3(+, \mathrm{CH}), 54.4(+, \mathrm{CH}), 47.4\left(13-\mathrm{C}_{\mathrm{q}}\right), 44.9(+, 5-\mathrm{CH}), 38.0\left(-, \mathrm{CH}_{2}\right)$, $36.7\left(-, \mathrm{CH}_{2}\right), 35.5\left(10-\mathrm{C}_{\mathrm{q}}\right), 35.2\left(-, \mathrm{CH}_{2}\right), 33.9(+, 8-\mathrm{CH}), 31.7\left(-, \mathrm{CH}_{2}\right), 31.5\left(-, \mathrm{CH}_{2}\right), 31.3\left(-, \mathrm{CH}_{2}\right)$, $28.4\left(-, 6-\mathrm{CH}_{2}\right), 21.0\left(-, 11-\mathrm{CH}_{2}\right), 16.5\left(+, 18-\mathrm{CH}_{3}\right), 12.1\left(+, 19-\mathrm{CH}_{3}\right) \mathrm{ppm} .-$ IR (ATR): $v=3314$ (br), $2922(\mathrm{w}), 2850$ (w), 1597 (vw), 1438 (w), 1416 (w), 1224 (vw), 1190 (w), 1119 (w), 1080 (w), 1049 (w), $1025(\mathrm{w}), 855(\mathrm{vw}), 802(\mathrm{w}), 754(\mathrm{vw}), 720(\mathrm{w}), 695(\mathrm{w}), 629(\mathrm{vw}), 540(\mathrm{~m}), 503(\mathrm{vw}), 406$ $(\mathrm{vw}) \mathrm{cm}^{-1}$. - MS (EI, $\left.70 \mathrm{eV}, 130^{\circ} \mathrm{C}\right): \mathrm{m} / z(\%)=351(65)[\mathrm{M}]^{++}, 336(100)\left[\mathrm{M}-\mathrm{CH}_{3}\right]^{+}, 277$ (71). HRMS (EI, $\left.\mathrm{C}_{24} \mathrm{H}_{33} \mathrm{ON}\right)$ : calc. $=351.2557$; found $=351.2556$. 


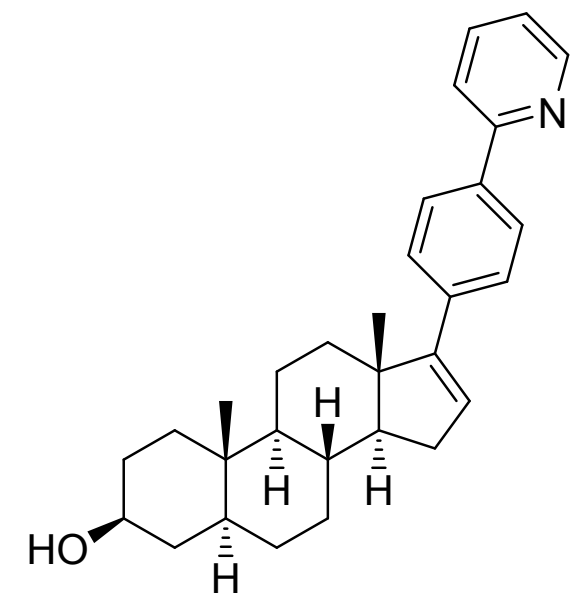

In a Schlenk tube, alkenyl iodide $4 \mathbf{b}(50 \mathrm{mg}, 125 \mu \mathrm{mol}$, 1.00 equiv.), crude 2-(4-tributylstannylphenyl)pyridine ${ }^{2}(83 \mathrm{mg}$, $187 \mu \mathrm{mol}, 1.50$ equiv.), $\mathrm{LiCl}$ (53 mg, $1.25 \mathrm{mmol}, 10.0$ equiv.) and $\mathrm{CuCl}$ (124 mg, $1.25 \mathrm{mmol}, 10.0$ equiv.) were dissolved in dry DMF $(3.0 \mathrm{~mL})$ under argon atmosphere. The reaction mixture was degassed using three freeze-pump-thaw cycles. Then $\mathrm{Pd}\left(\mathrm{PPh}_{3}\right)_{4}(29 \mathrm{mg}, 25 \mu \mathrm{mol}, 20 \mathrm{~mol} \%$ ) was added and the reaction mixture was stirred at $80^{\circ} \mathrm{C}$ for $60 \mathrm{~h}$. After cooling to room temperature an aqueous solution of $\mathrm{KF}$ ( $3 \mathrm{M}, 4.00$ equiv.) was added and stirred for $30 \mathrm{~min}$, followed by filtration over

Celite ${ }^{\circledR}$ (dichloromethane as eluent). The organic phase was washed with aqueous saturated $\mathrm{NH}_{4} \mathrm{Cl}$ solution $(50 \mathrm{~mL})$ and extracted with dichloromethane $(2 \times 50 \mathrm{~mL})$. The combined organic layers were washed with brine $(100 \mathrm{~mL})$, dried over $\mathrm{Na}_{2} \mathrm{SO}_{4}$ and filtered. The solvent was removed under reduced pressure and, after flash column chromatography on silica gel (cyclohexane/ethyl acetate, 4:1), the title compound $\mathbf{5 c}$ was obtained as a colorless solid $(34 \mathrm{mg}, 80.0 \mu \mathrm{mol}, 64 \%)$. For biological tests the compound was washed multiple times with $n$-hexane.

$\boldsymbol{R}_{\boldsymbol{f}}=0.14(\mathrm{cHex} / \mathrm{EtOAc}, 3: 1) .{ }^{1} \mathbf{H}$ NMR $\left(400 \mathrm{MHz}, \mathrm{CDCl}_{3}\right): \delta=8.61(\mathrm{dt}, J=4.8,1.6 \mathrm{~Hz}, 1 \mathrm{H}$, $\left.\mathrm{N}=\mathrm{C} H_{\text {Pyr }}\right), 7.85\left(\mathrm{~d}, J=8.5 \mathrm{~Hz}, 2 \mathrm{H}, \mathrm{C} H_{\mathrm{Ph}}\right), 7.70-7.74\left(\mathrm{~m}, 1 \mathrm{H}, \mathrm{C} H_{\text {Pyr }}\right), 7.67-7.64\left(\mathrm{~m}, 2 \mathrm{H}, \mathrm{C} H_{\text {Pyr }}\right), 7.41(\mathrm{~d}$, $\left.J=8.5 \mathrm{~Hz}, 2 \mathrm{H}, \mathrm{C} H_{\mathrm{Ph}}\right), 7.14\left(\mathrm{ddd}, J=6.1,4.8,2.4 \mathrm{~Hz}, 1 \mathrm{H}, \mathrm{CH}_{\mathrm{Pyr}}\right), 5.92(\mathrm{dd}, J=3.3,1.8 \mathrm{~Hz}, 1 \mathrm{H},=\mathrm{CH})$, $3.52(\mathrm{tt}, J=9.6,4.3 \mathrm{~Hz}, 1 \mathrm{H}, \mathrm{CHOH}), 2.15\left(\mathrm{ddd}, J=15.7,6.43 .3 \mathrm{~Hz}, 1 \mathrm{H},=\mathrm{CHCH}_{2}\right), 2.07-1.89(\mathrm{~m}, 2 \mathrm{H}$, $\left.=\mathrm{CHCH}_{2}+\mathrm{CH}_{2}\right), 1.75-1.37\left(\mathrm{~m}, 9 \mathrm{H}, 5\right.$ different $\left.\mathrm{CH}_{2}, 8-\mathrm{CH}+14-\mathrm{CH}\right), 1.32-1.17(\mathrm{~m}, 4 \mathrm{H}, 3$ different $\left.\mathrm{CH}_{2}\right), 1.17-1.11(\mathrm{~m}, 1 \mathrm{H}, 5-\mathrm{CH}), 1.00-0.88\left(\mathrm{~m}, 2 \mathrm{H}, 2\right.$ different $\left.\mathrm{CH}_{2}\right), 0.98\left(\mathrm{~s}, 3 \mathrm{H}, \mathrm{CH}_{3}\right), 0.80(\mathrm{~s}, 3 \mathrm{H}$, $\left.\mathrm{CH}_{3}\right), 0.61-0.77$ (m, 1H, 9-CH) ppm. - ${ }^{13} \mathbf{C}$ NMR $\left(100 \mathrm{MHz}, \mathrm{CDCl}_{3}\right): \delta=157.1\left(C_{\mathrm{q}}=\mathrm{N}\right), 154.3\left(C_{\mathrm{q}}=\mathrm{CH}\right)$, $149.5\left(+, C \mathrm{H}_{\mathrm{Pyr}}\right), 137.9\left(C_{\mathrm{q}}=\mathrm{CH}_{\mathrm{Ar}}\right), 137.4\left(C_{\mathrm{q}}=\mathrm{CH}_{\mathrm{Ar}}\right), 136.5\left(+, C \mathrm{H}_{\mathrm{Pyr}}\right), 127.7(+,=C \mathrm{H}), 126.8(+$, $\left.2 \times \mathrm{CH}_{\mathrm{Ph}}\right), 126.4\left(+, 2 \times C \mathrm{H}_{\mathrm{Ph}}\right), 121.7\left(+, \mathrm{CH}_{\mathrm{Pyr}}\right), 120.1\left(+, C \mathrm{H}_{\mathrm{Pyr}}\right), 71.1(+, \mathrm{CHOH}), 57.4(+, \mathrm{CH}), 54.5$ $(+, C H), 47.3\left(13-C_{\mathrm{q}}\right), 44.9(+, 5-C \mathrm{H}), 38.2\left(-, \mathrm{CH}_{2}\right), 36.7\left(-, \mathrm{CH}_{2}\right), 35.5\left(10-C_{\mathrm{q}}\right), 35.4\left(-, \mathrm{CH}_{2}\right), 33.9$ $(+, 8-\mathrm{CH}), 31.8\left(-, \mathrm{CH}_{2}\right), 31.5\left(-, \mathrm{CH}_{2}\right), 31.4\left(-, \mathrm{CH}_{2}\right), 28.5\left(-, \mathrm{CH}_{2}\right), 21.1\left(-, \mathrm{CH}_{2}\right), 16.6\left(+, \mathrm{CH}_{3}\right), 12.2$ (+, $\left.\mathrm{CH}_{3}\right)$ ppm. - IR (ATR): v = 3412 (vw), 2919 (w), 2847 (w), $1584(\mathrm{w}), 1463$ (w), 1434 (w), 1378 (w), $1299(\mathrm{w}), 1157(\mathrm{vw}), 1135(\mathrm{vw}), 1039(\mathrm{w}), 989(\mathrm{vw}), 846(\mathrm{w}), 781(\mathrm{~m}), 751(\mathrm{w}), 631(\mathrm{w}), 494(\mathrm{w})$, $407(\mathrm{w}) \mathrm{cm}^{-1}$. - MS (EI, $\left.70 \mathrm{eV}, 200{ }^{\circ} \mathrm{C}\right): \mathrm{m} / z(\%)=427(20)[\mathrm{M}]^{++}, 412(13)\left[\mathrm{M}_{-} \mathrm{CH}_{3}\right]^{+}, 308(100)$. HRMS (EI, $\left.\mathrm{C}_{30} \mathrm{H}_{37} \mathrm{ON}\right)$ : calc. $=427.2870$; found $=427.2870$. 


\subsection{Synthetic procedures for the ruthenium(II) and iridium(III) complexes}

General procedure for the cyclometalation

Under argon atmosphere, the ligand (2.00 equiv.), $\left[\mathrm{Ru}(p \text {-cymene }) \mathrm{Cl}_{2}\right]_{2}\left(1.00\right.$ equiv.) or $\left[\mathrm{Ir} \mathrm{Cp} * \mathrm{Cl}_{2}\right]_{2}$ and KOAc (4.00 equiv.) were dissolved in dry $\mathrm{MeOH}$ or $\mathrm{CH}_{2} \mathrm{Cl}_{2}$ and stirred at room temperature for $24 \mathrm{~h}$. The suspension was concentrated and the residue was purified by flash column chromatography on silica gel (cyclohexane/ethyl acetate) to obtain the corresponding cyclometalated complex as a yellow to orange solid.

\section{[5-Bromo-2-(2-pyridinyl- $\kappa N)$ phenyl- $\kappa C$ Chlorido( $\eta^{6}$-para-cymene) ruthenium(II) (6)}

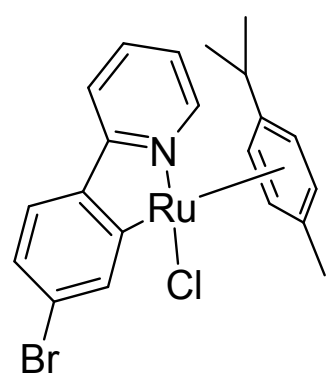

Following the general procedure, $\left[\mathrm{Ru}(p \text {-cymene }) \mathrm{Cl}_{2}\right]_{2}(306 \mathrm{mg}, 0.50 \mathrm{mmol}$, 1.00 equiv.), 2-(4-bromophenyl)pyridine ( $234 \mathrm{mg}, 1.00 \mathrm{mmol}, 2.00$ equiv.) and KOAc (196 mg, $2.00 \mathrm{mmol}, 4.00$ equiv.) were dissolved in dry $\mathrm{MeOH}$ $(10 \mathrm{~mL})$ and stirred $24 \mathrm{~h}$ at room temperature. The suspension was concentrated and then purified via flash column chromatography on silica gel (cyclohexane/ethyl acetate, 1:1) to afford the title compound $\mathbf{6}$ as a yellow solid (336 mg, $0.667 \mathrm{mmol}, 67 \%$ ).

$\boldsymbol{R}_{\mathbf{f}}=0.14(c \mathrm{Hex} /$ EtOAc, $1: 1) \cdot-{ }^{1} \mathbf{H}$ NMR $\left(500 \mathrm{MHz}, \mathrm{CDCl}_{3}\right): \delta=9.13\left(\mathrm{~d}, J=5.7 \mathrm{~Hz}, 1 \mathrm{H}, 3-\mathrm{C}_{\text {Pyr }}\right), 8.21$ $\left(\mathrm{d}, J=1.9 \mathrm{~Hz}, 1 \mathrm{H}, 6-\mathrm{CH}_{\mathrm{Ar}}\right), 7.67-7.56\left(\mathrm{~m}, 2 \mathrm{H}, \mathrm{C} H_{\mathrm{Ar}}\right), 7.39\left(\mathrm{~d}, J=8.2 \mathrm{~Hz}, 1 \mathrm{H}, \mathrm{C} H_{\mathrm{Ar}}\right), 7.09(\mathrm{dd}, J=8.2$, $\left.1.9 \mathrm{~Hz}, 1 \mathrm{H}, \mathrm{C} H_{\mathrm{Ar}}\right), 7.04-6.98\left(\mathrm{~m}, 1 \mathrm{H}, \mathrm{C} H_{\mathrm{Ar}}\right), 5.51\left(\mathrm{~d}, J=6.0 \mathrm{~Hz}, 2 \mathrm{H}, \mathrm{CH}_{\text {cymene }}\right), 5.14$ (d, $J=6.0 \mathrm{~Hz}$, $\left.1 \mathrm{H}, \mathrm{CH}_{\text {cymene }}\right), 4.93$ (d, $\left.J=5.9 \mathrm{~Hz}, 1 \mathrm{H}, \mathrm{CH}_{\text {cymene }}\right), 2.36$ (hept, $\left.J=6.9 \mathrm{~Hz}, 1 \mathrm{H}, \mathrm{CH}\left(\mathrm{CH}_{3}\right)_{2}\right), 2.00(\mathrm{~s}, 3 \mathrm{H}$, $\left.\mathrm{C}_{\mathrm{q}, \text { cymene }} \mathrm{CH}_{3}\right), 0.91\left(\mathrm{~d}, J=6.9 \mathrm{~Hz}, 3 \mathrm{H}, \mathrm{CHCH}_{3}\right), 0.81\left(\mathrm{~d}, J=6.9 \mathrm{~Hz}, 3 \mathrm{H}, \mathrm{CHCH}_{3}\right)$ ppm. $-{ }^{13} \mathbf{C ~ N M R}$ $\left(126 \mathrm{MHz}, \mathrm{CDCl}_{3}\right): \delta=183.4\left(C_{\mathrm{q}}-\mathrm{Ru}\right), 164.5\left(C_{\mathrm{q}} \mathrm{N}\right), 154.7\left(+, \mathrm{CH}_{\text {aryl }}\right), 142.1\left(C_{\mathrm{q}}\right), 141.3\left(+, \mathrm{CH}_{\text {aryl }}\right)$, $136.9\left(+, C \mathrm{H}_{\text {aryl }}\right), 125.6\left(+, C \mathrm{H}_{\text {aryl }}\right), 125.0\left(+, C \mathrm{H}_{\text {aryl }}\right), 124.3\left(C_{\mathrm{q}}\right), 121.8\left(+, C \mathrm{H}_{\text {aryl }}\right), 119.0\left(+, C \mathrm{H}_{\text {aryl }}\right)$, $101.4\left(C_{\mathrm{q}}\right), 100.8\left(C_{\mathrm{q}}\right), 91.0\left(+, C \mathrm{H}_{\text {cymene }}\right), 89.4\left(+, C \mathrm{H}_{\text {cymene }}\right), 84.8\left(+, C \mathrm{H}_{\text {cymene }}\right), 82.1\left(+, C \mathrm{H}_{\text {cymene }}\right), 30.8$ $\left(+, \mathrm{CH}\left(\mathrm{CH}_{3}\right)_{2}\right), 22.5\left(+, \mathrm{CHCH}_{3}\right), 21.8\left(+, \mathrm{CHCH}_{3}\right), 18.8\left(+, \mathrm{C}_{\mathrm{q}, \mathrm{Ar}} \mathrm{CH}_{3}\right)$ ppm. - IR (ATR): $v=2953(\mathrm{vw})$, 1732 (vw), 1601 (w), 1566 (w), 1533 (w), 1470 (w), 1414 (w), 1380 (w), 1293 (vw), 1257 (w), 1095 (vw), 1067 (vw), $1045(\mathrm{w}), 1007$ (w), 860 (w), 768 (m), 712 (vw), 683 (w), 566 (vw) cm-1 . MS (FAB, 3-NBA): $m / z(\%)=510 / 509 / 508 / 507 / 506 / 505 / 504 / 503 / 502 / 501 / 500 / 499[\mathrm{M}]^{+}, 472 / 471 / 470 / 469 / 468 /$ 467/466/465/464/463/462 [M-Cl] $]^{+}$- HRMS (FAB, $\mathrm{C}_{21} \mathrm{H}_{21} \mathrm{~N}^{79} \mathrm{Br}^{35} \mathrm{Cl}^{102} \mathrm{Ru},[\mathrm{M}]^{+}$): calc. = 502.9589; found $=502.9590$. 


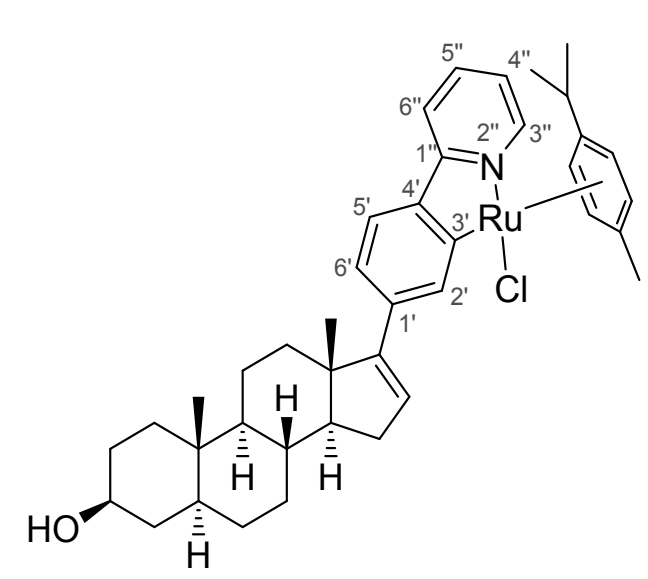

According to the general procedure (3 $\beta, 5 \alpha)$-17-(4' -pyridin2 '-ylphenyl)androst-16-en-3-ol (5c) (100 mg, $234 \mu \mathrm{mol})$, $\left[\mathrm{Ru}(p \text {-cymene }) \mathrm{Cl}_{2}\right]_{2}(72 \mathrm{mg}, 117 \mu \mathrm{mol})$ and KOAc $(46 \mathrm{mg}$, $468 \mu \mathrm{mol})$ stirred in dry methanol $(10 \mathrm{~mL})$ for $20 \mathrm{~h}$ at room temperature. After flash column chromatography on silica gel ( $c$ Hex/EtOAc/ $\left.\mathrm{NEt}_{3}, \quad 200: 200: 1 \rightarrow 0: 200: 1\right)$ the ruthenium(II) complex 7 was obtained as a yellow solid (48 $\mathrm{mg}, 83.0 \mu \mathrm{mol}, 35 \%)$ consisting of an inseparable mixture of its diastereomers $(d r=1: 1$.$) . An unambiguous$ assignment of the signals in order to definite diastereomer was not possible even with 2D NMR experiments. Therefore, the signals for the diastereomeric mixture are given, same signals are stated with "and" if clearly distinguishable.

$\boldsymbol{R}_{\boldsymbol{f}}=0.31\left(\mathrm{EtOAc} / \mathrm{NEt}_{3}, 200: 1\right) .-{ }^{1} \mathbf{H}$ NMR $\left(600 \mathrm{MHz}, \mathrm{CDCl}_{3}\right): \delta=9.20\left(\mathrm{~d}, J=5.6 \mathrm{~Hz}, 1 \mathrm{H}, 3^{\prime \prime}-\mathrm{CH} H_{\mathrm{Ar}} \mathrm{N}\right)$, $8.16\left(\mathrm{~s}, 1 \mathrm{H}, 2^{\prime}-\mathrm{CH}_{\mathrm{Ar}}\right), 7.68-7.59\left(\mathrm{~m}, 2 \mathrm{H}, 5^{\prime \prime}-\mathrm{CH}_{\mathrm{Ar}}+6^{\prime \prime}-\mathrm{C} H_{\mathrm{Ar}}\right) 7.52\left(\mathrm{~d}, J=8.0 \mathrm{~Hz}, 1 \mathrm{H}, 5^{\prime}-\mathrm{C} H_{\mathrm{Ar}}\right), 7.04$ (d, $\left.J=8.2 \mathrm{~Hz}, 1 \mathrm{H}, 6{ }^{\prime}-\mathrm{C} H_{\mathrm{Ar}}\right), 7.01\left(\mathrm{ddd}, J=7.1,5.7,1.7 \mathrm{~Hz}, 1 \mathrm{H}, 4^{\prime \prime}{ }^{-}-\mathrm{CH}_{\mathrm{Ar}}\right), 6.03$ (ddd, $J=10.3,3.3$, $1.8 \mathrm{~Hz}, 1 \mathrm{H},=\mathrm{C} H), 5.57\left(\mathrm{~d}, J=5.9 \mathrm{~Hz}, 1 \mathrm{H}, \mathrm{C} H_{\text {cymene }}\right), 5.49$ (dd, $\left.J=17.1,5.8 \mathrm{~Hz}, 1 \mathrm{H}, \mathrm{C} H_{\text {cymene }}\right), 5.17$ (dd, $J=5.2 \mathrm{~Hz}, 1 \mathrm{H}, \mathrm{C} H_{\text {cymene }}$ ), 5.00 (dd, $J=8.2,6.4 \mathrm{~Hz}, 1 \mathrm{H}, \mathrm{C} H_{\text {cymene }}$ ), 3.60 (tt, $\left.J=10.6,4.8 \mathrm{~Hz}, 1 \mathrm{H}, \mathrm{CHOH}\right)$, 2.48 (hep, $\left.J=6.9 \mathrm{~Hz}, 1 \mathrm{H}, \mathrm{CH}\left(\mathrm{CH}_{3}\right)_{2}\right), 2.23\left(\mathrm{dtd}, J=15.6,6.2,3.3 \mathrm{~Hz}, 1 \mathrm{H}, \mathrm{CH}_{2}\right), 2.21-2.13(\mathrm{~m}, 1 \mathrm{H}$, $\left.\mathrm{CH}_{2}\right), 2.08-2.02\left(\mathrm{~m}, 1 \mathrm{H}, \mathrm{CH}_{2}\right), 2.01$ and 1.99 each (s, 3H, $\left.\mathrm{C}_{\mathrm{q} \text {,cymene }} \mathrm{CH}_{3}\right), 1.83-1.23(\mathrm{~m}, 13 \mathrm{H}, 7$ different $\left.\mathrm{CH}_{2}+8-\mathrm{CH}+14-\mathrm{CH}\right), 1.21-1.13(\mathrm{~m}, 1 \mathrm{H}, 5-\mathrm{CH}), 1.12$ and 1.08 each $\left(\mathrm{s}, 3 \mathrm{H}, 18-\mathrm{CH}_{3}\right), 1.06-1.00(\mathrm{~m}$, $2 \mathrm{H}, 2$ different $\left.\mathrm{CH}_{2}\right), 1.01\left(\mathrm{dd}, J=6.9 \mathrm{~Hz}, 3 \mathrm{H}, \mathrm{CH}\left(\mathrm{CH}_{3}\right)_{2}\right), 0.92$ and 0.90 each $(\mathrm{d}, J=6.9 \mathrm{~Hz}, 1.5 \mathrm{H}$, $\left.\mathrm{CH}\left(\mathrm{CH}_{3}\right)_{2}\right), 0.88\left(\mathrm{~s}, 3 \mathrm{H}, 19-\mathrm{CH}_{3}\right), 0.83-0.73(\mathrm{~m}, 1 \mathrm{H}, 9-\mathrm{CH}) \mathrm{ppm} .-{ }^{13} \mathbf{C}$ NMR $\left(151 \mathrm{MHz}, \mathrm{CDCl}_{3}\right)$ : $\delta=180.5\left(C_{\mathrm{q}}-\mathrm{Ru}\right), 165.1\left(1^{\prime \prime}-C_{\mathrm{q}} \mathrm{N}\right), 155.7$ and 155.5 each $\left(17-C_{\mathrm{q}}\right), 154.4\left(+, 3^{\prime \prime}{ }^{\prime}-C_{\mathrm{Ar}}\right), 141.9$ and 141.9 each $\left(4^{\prime}-C_{\mathrm{q}}\right), 137.9\left(1^{\prime}-C_{\mathrm{q}}\right), 137.4$ and 137.3 each $\left(+, 2^{\prime}-C \mathrm{H}_{\mathrm{Ar}}\right), 136.4\left(+, 5^{\prime \prime}-C \mathrm{H}_{\mathrm{Ar}}\right), 127.3(+,=C \mathrm{H})$, 123.2 and 123.1 each $\left(+, 5^{\prime}-\mathrm{CH}_{\mathrm{Ar}}\right), 121.4$ and 121.2 each $\left(+, 6^{\prime}\right.$-or $\left.4^{\prime \prime}{ }^{\prime}-\mathrm{CH}_{\mathrm{Ar}}\right), 120.9$ and 120.9 each $(+$, $6^{\prime}$ - or $\left.4^{\prime \prime}-\mathrm{CH}_{\mathrm{Ar}}\right), 118.5$ and 118.5 each $\left(+, 6^{\prime \prime}{ }^{\prime}-\mathrm{CH}_{\mathrm{Ar}}\right), 100.9$ and 100.8 each $\left(\mathrm{C}_{\mathrm{q}, \text { cymene }} \mathrm{CH}\left(\mathrm{CH}_{3}\right)_{2}\right), 99.7$ and 99.2 each $\left(C_{\text {q,cymene }} \mathrm{CH}_{3}\right), 90.2$ and 90.1 each $\left(+, \mathrm{CH}_{\text {cymene }}\right), 89.9$ and 89.8 each $\left(+, \mathrm{CH}_{\text {cymene }}\right), 83.7$ and 83.6 each $\left(+, C \mathrm{H}_{\text {cymene }}\right), 83.1$ and 82.6 each $\left(+, C \mathrm{H}_{\text {cymene }}\right), 71.3$ and 71.2 each $(+, 3-\mathrm{CHOH}), 57.6$ and 57.6 each $(+, C H), 54.7$ and 54.6 each $(+, C H), 47.6$ and $47.4\left(13-C_{\mathrm{q}}\right), 45.0$ and 45.0 each $(+, C H)$, $38.2\left(-, \mathrm{CH}_{2}\right), 36.8\left(-, \mathrm{CH}_{2}\right), 35.9$ and 35.6 each $\left(-, \mathrm{CH}_{2}+10-\mathrm{C}_{\mathrm{q}}\right), 34.1$ and 34.0 each $(+, \mathrm{CH}), 31.9$ and 31.9 each $\left(-, \mathrm{CH}_{2}\right), 31.6$ and 31.6 each $\left(-, \mathrm{CH}_{2}\right), 31.5\left(-, \mathrm{CH}_{2}\right), 30.7\left(+, \mathrm{CH}\left(\mathrm{CH}_{3}\right)_{2}\right), 28.7\left(-, \mathrm{CH}_{2}\right), 22.6$ and 22.5 each $\left(+, \mathrm{CH}\left(\mathrm{CH}_{3}\right)_{2}\right), 21.6$ and 21.6 each $\left(+, \mathrm{CH}\left(\mathrm{CH}_{3}\right)_{2}\right), 21.3\left(-, 11-\mathrm{CH}_{2}\right), 18.6$ and 18.6 each $\left.\left(+, \mathrm{C}_{\mathrm{q}, \text { cymene }} \mathrm{CH}_{3}\right)_{2}\right), 17.0$ and 17.0 each $\left.\left.\left(+, 18-\mathrm{CH}_{3}\right)_{2}\right), 12.3\left(+, 19-\mathrm{CH}_{3}\right)_{2}\right)$ ppm. - IR (ATR): $v=3401$ (br), $2917(\mathrm{w}), 2848(\mathrm{w}), 1601(\mathrm{vw}), 1576(\mathrm{vw}), 1558(\mathrm{vw}), 1471(\mathrm{w}), 1378(\mathrm{vw}), 1316(\mathrm{vw}), 1158(\mathrm{vw})$, 1079 (vw), 1037 (vw), 1007 (vw), 957 (vw), 918 (vw), 828 (vw), 810 (vw), 774 (w), 724 (w), $642(\mathrm{vw})$, 
498 (vw) $\mathrm{cm}^{-1} . \quad-$ MS (FAB, 3-NBA): $m / z \quad(\%)=694 / 695 / 696 / 697 / 698 / 699 / 700 \quad[\mathrm{M}]^{+}$, 658/659/660/662/663/664/665 [M-Cl] ${ }^{+}, 599 / 600 / 601 / 602 / 603 / 604 / 605,154$ (100). - HRMS (FAB, $\left.\mathrm{C}_{40} \mathrm{H}_{50} \mathrm{ON}^{35} \mathrm{Cl}^{102} \mathrm{Ru},[\mathrm{M}+\mathrm{H}]^{+}\right)$: calc $=697.2619$; found $=697.2620$.

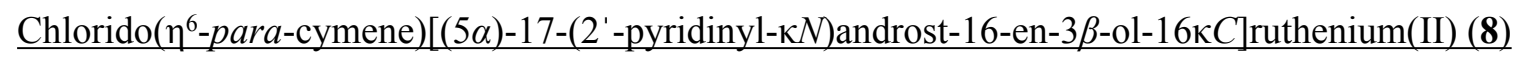

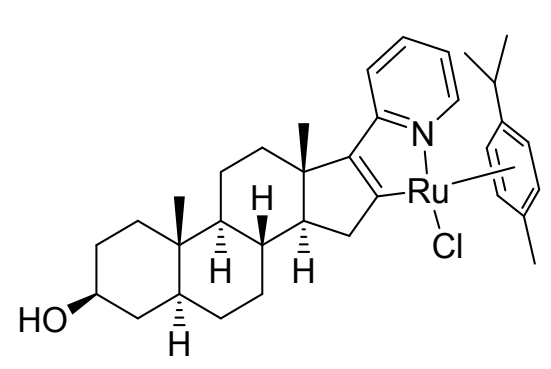

According to the general procedure, $(3 \beta, 5 \alpha)-17-\left(2^{\prime}-\right.$ pyridinyl)androst-16-en-3-ol (5a) $(33 \mathrm{mg}, 94.4 \mu \mathrm{mol}),[\mathrm{Ru}(p$ cymene $\left.) \mathrm{Cl}_{2}\right]_{2}(29 \mathrm{mg}, 47.2 \mu \mathrm{mol})$ and KOAc $(19 \mathrm{mg}, 189 \mu \mathrm{mol})$ in dry methanol $(1.0 \mathrm{~mL})$ were stirred $20 \mathrm{~h}$ at room temperature. After flash column chromatography on silica gel ( $c \mathrm{Hex} / \mathrm{EtOAc} /$ $\left.\mathrm{NEt}_{3}, 1: 1: 0.05 \rightarrow 0: 1: 0.05\right)$ the two diastereo-meric ruthenium(II) complexes 8 were obtained as yellow solids $(\mathrm{F} 1=(\boldsymbol{R}-\mathbf{R u})-\mathbf{8}: 16 \mathrm{mg}, 25.7 \mu \mathrm{mol}, 27 \%$; F2 $=(\boldsymbol{S}$-Ru) $)-8: 14$ $\mathrm{mg}, 22.5 \mu \mathrm{mol}, 24 \%)$.

\section{$\underline{(R-R u)-8^{*}}$}

$\boldsymbol{R}_{\boldsymbol{f}}=0.63\left(\mathrm{EtOAc} / \mathrm{NEt}_{3}, 200: 1\right) .{ }^{1} \mathbf{H}$ NMR $\left(500 \mathrm{MHz}, \mathrm{CDCl}_{3}\right): \delta=9.09\left(\mathrm{dd}, J=5.8,1.5 \mathrm{~Hz}, 1 \mathrm{H}, 3^{\prime}-\right.$ $\mathrm{C} H \mathrm{~N}), 7.42\left(\mathrm{td}, J=7.8,1.6 \mathrm{~Hz}, 1 \mathrm{H}, 5^{\prime}-\mathrm{CH}\right), 7.09\left(\mathrm{~d}, J=8.1 \mathrm{~Hz}, 1 \mathrm{H}, 6^{\prime}-\mathrm{CH}\right), 6.68(\mathrm{t}, J=6.5 \mathrm{~Hz}, 1 \mathrm{H}$, 4'-CH), 5.54 (d, $\left.J=5.9 \mathrm{~Hz}, 1 \mathrm{H}, \mathrm{CH}_{\text {cymene }}\right), 5.51$ (d, $\left.J=5.9 \mathrm{~Hz}, 1 \mathrm{H}, \mathrm{C}_{\text {cymene }}\right), 5.21$ (d, $J=5.9 \mathrm{~Hz}, 1 \mathrm{H}$, $\left.\mathrm{C}_{\text {cymene }}\right), 4.93$ (d, $\left.J=5.7 \mathrm{~Hz}, 1 \mathrm{H}, \mathrm{C} H_{\text {cymene }}\right), 3.60$ (tt, $\left.J=10.6,4.6 \mathrm{~Hz}, 1 \mathrm{H}, \mathrm{CHOH}\right), 3.11$ (dd, $\left.{ }^{2} J=16.2 \mathrm{~Hz},{ }^{3} J=4.8 \mathrm{~Hz}, 1 \mathrm{H}, 15-\mathrm{CH}_{2}{ }^{\mathrm{a}}\right), 2.50\left(\mathrm{dd}, J=16.3,11.4 \mathrm{~Hz}, 1 \mathrm{H}, 15-\mathrm{CH}_{2}{ }^{\mathrm{b}}\right), 2.41(\mathrm{p}, J=6.9 \mathrm{~Hz}$, $\left.1 \mathrm{H}, \mathrm{CH}\left(\mathrm{CH}_{3}\right)_{2}\right), 2.19\left(\mathrm{dd}, J=8.1,2.7 \mathrm{~Hz}, 1 \mathrm{H}, \mathrm{CH}_{2}\right), 2.01\left(\mathrm{~s}, 3 \mathrm{H}, \mathrm{C}_{\mathrm{q}, \text { cymene }} \mathrm{CH}_{3}\right), 2.19$ (dd, $J=8.1,2.7 \mathrm{~Hz}$, $\left.1 \mathrm{H}, \mathrm{CH}_{2}\right), 1.89-1.80\left(\mathrm{~m}, 2 \mathrm{H}, 2\right.$ different $\left.\mathrm{CH}_{2}\right), 1.76-1.57\left(\mathrm{~m}, 5 \mathrm{H}, 3\right.$ different $\left.\mathrm{CH}_{2}+\mathrm{CH}+\mathrm{CH}\right), 1.52-$ $1.28\left(\mathrm{~m}, 6 \mathrm{H}, 5\right.$ different $\left.\mathrm{CH}_{2}\right), 1.22-1.11\left(\mathrm{~m}, 2 \mathrm{H}, \mathrm{CH}_{2}+\mathrm{CH}\right), 1.00\left(\mathrm{~d},{ }^{3} \mathrm{~J}=5.1 \mathrm{~Hz}, 3 \mathrm{H}, \mathrm{CH}\left(\mathrm{CH}_{3}\right)_{2}\right), 0.98$ $\left(\mathrm{d},{ }^{3} \mathrm{~J}=5.3 \mathrm{~Hz}, 3 \mathrm{H}, \mathrm{CH}\left(\mathrm{CH}_{3}\right)_{2}\right), 0.86\left(\mathrm{~s}, 3 \mathrm{H}, 19-\mathrm{CH}_{3}\right), 0.85-0.78(\mathrm{~m}, 1 \mathrm{H}, \mathrm{CH}), 0.84\left(\mathrm{~s}, 3 \mathrm{H}, 18-\mathrm{CH}_{3}\right) \mathrm{ppm}$. ${ }^{13} \mathrm{C}$ NMR (126 MHz, $\left.\mathrm{CDCl}_{3}\right): \delta=208.7\left(16-C_{\mathrm{q}}-\mathrm{Ru}\right), 163.4\left(1^{\prime}-C_{\mathrm{q}} \mathrm{N}\right), 154.7\left(+, 3^{\prime}-\mathrm{CH}_{\mathrm{Ar}} \mathrm{N}\right), 150.4$ $\left(17-C_{\mathrm{q}}\right), 135.8\left(+, 5^{\prime}-C \mathrm{H}_{\mathrm{Ar}}\right), 117.8\left(+, 6^{\prime}-\mathrm{CH}_{\mathrm{Ar}}\right), 116.4\left(+, 4^{\prime}-C \mathrm{H}_{\mathrm{Ar}}\right), 99.7\left(C_{\mathrm{q}, \text { cymene }}\right), 99.1\left(C_{\mathrm{q}, \text { cymene }}\right), 90.0$ (+, $\left.\mathrm{CH}_{\text {cymene }}\right), 87.3$ (+, $\left.\mathrm{CH}_{\text {cymene }}\right), 85.5$ (+, $\left.\mathrm{CH}_{\text {cymene }}\right), 81.2,\left(+, C \mathrm{H}_{\text {cymene }}\right), 71.3(+, 3-\mathrm{CHOH}), 58.5(+, 14-$ $\mathrm{CH}), 55.2(+, 9-\mathrm{CH}), 45.1(+, 5-\mathrm{CH}), 44.9\left(13-\mathrm{C}_{\mathrm{q}}\right), 44.8\left(-, \mathrm{CH}_{2}\right), 38.2\left(-, \mathrm{CH}_{2}\right), 36.8\left(-, \mathrm{CH}_{2}\right), 36.2(-$, $\left.\mathrm{CH}_{2}\right), 35.7\left(10-\mathrm{C}_{\mathrm{q}}\right), 33.8(+, 8-\mathrm{CH}), 32.2\left(-, \mathrm{CH}_{2}\right), 31.4\left(-, \mathrm{CH}_{2}\right), 30.9\left(+, \mathrm{CH}\left(\mathrm{CH}_{3}\right)_{2}\right), 28.7\left(-, \mathrm{CH}_{2}\right)$, $22.2\left(+, \mathrm{CH}\left(\mathrm{CH}_{3}\right)_{2}\right), 22.1\left(+, \mathrm{CH}\left(\mathrm{CH}_{3}\right)_{2}\right), 21.3\left(-, \mathrm{CH}_{2}\right), 18.7\left(+, \mathrm{C}_{\mathrm{q}, \text { cymene }}\left(\mathrm{CH}_{3}\right)_{2}\right), 16.6\left(+, 18-\mathrm{CH}_{3}\right), 12.3$ $\left(+, 19-\mathrm{CH}_{3}\right)$ ppm. - IR (ATR): v = 3367 (vw), 2918 (m), 2849 (m), 1736 (w), $1662(\mathrm{w}), 1596(\mathrm{w}), 1501$ (w), 1465 (m), 1376 (w), 1241 (w), 1151 (w), 1080 (w), 1040 (w), 961 (w), 842 (vw), 776 (w), 745 (w) $\mathrm{cm}^{-1}$. - MS (+FAB, 3-NBA): $m / z \quad(\%)=618 / 619 / 620 / 621 / 622 / 623 / 624 / 625 \quad[\mathrm{M}+\mathrm{H}]^{+}$, 581/582/583/584/585/586/587/588 [M-Cl] . - HRMS (+FAB, $\left.\mathrm{C}_{34} \mathrm{H}_{47} \mathrm{O}_{1} \mathrm{~N}_{1}{ }^{35} \mathrm{Cl}_{1}{ }^{102} \mathrm{Ru}_{1}, \quad[\mathrm{M}+\mathrm{H}]^{+}\right)$: calc $=622.2384$; found $=622.2385$. 


\section{$\underline{(S-\mathrm{Ru})-\mathbf{8}^{*}}$}

$\boldsymbol{R}_{\boldsymbol{f}}=0.51\left(\mathrm{EtOAc}_{\mathrm{NEt}}, 200: 1\right) .{ }^{1} \mathbf{H}$ NMR $\left(500 \mathrm{MHz}, \mathrm{CDCl}_{3}\right): \delta=9.07\left(\mathrm{~d}, J=5.8 \mathrm{~Hz}, 1 \mathrm{H}, 3^{\prime}-\mathrm{C} H \mathrm{~N}\right)$, 7.41 (t, $\left.J=7.6 \mathrm{~Hz}, 1 \mathrm{H}, 5^{\prime}-\mathrm{CH}\right), 7.07$ (d, $\left.J=8.0 \mathrm{~Hz}, 1 \mathrm{H}, 6{ }^{\prime}-\mathrm{CH}\right), 6.68$ (t, $\left.J=6.5 \mathrm{~Hz}, 1 \mathrm{H}, 4^{\prime}-\mathrm{CH}\right), 5.57$ (d, $\left.J=6.0 \mathrm{~Hz}, 1 \mathrm{H}, \mathrm{CH}_{\text {cymene }}\right), 5.47$ (d, $\left.J=5.7 \mathrm{~Hz}, 1 \mathrm{H}, \mathrm{CH}_{\text {cymene }}\right), 5.17$ (d, $\left.J=6.0 \mathrm{~Hz}, 1 \mathrm{H}, \mathrm{CH}_{\text {cymene }}\right), 4.88$ (d, $J=5.7 \mathrm{~Hz}, 1 \mathrm{H}, \mathrm{CH}_{\text {cymene }}$ ), 3.57 (tt, $\left.J=10.5,4.7 \mathrm{~Hz}, 1 \mathrm{H}, \mathrm{CHOH}\right), 2.98$ (dd, $J=16.1,6.4 \mathrm{~Hz}, 1 \mathrm{H}, 15-$ $\left.\mathrm{CH}_{2}{ }^{\mathrm{a}}\right), 2.84\left(\mathrm{dd}, J=16.1,11.3 \mathrm{~Hz}, 1 \mathrm{H}, 15-\mathrm{CH}_{2}{ }^{\mathrm{b}}\right), 2.41-2.29\left(\mathrm{~m}, 1 \mathrm{H}, \mathrm{CH}\left(\mathrm{CH}_{3}\right)_{2}\right), 2.11(\mathrm{dd}, J=11.1$, $\left.4.1 \mathrm{~Hz}, 1 \mathrm{H}, \mathrm{CH}_{2}\right), 2.03\left(\mathrm{~s}, 3 \mathrm{H}, \mathrm{C}_{\mathrm{q}, \text { cymene }} \mathrm{CH}_{3}\right), 1.84-1.53\left(\mathrm{~m}, 8 \mathrm{H}, 6\right.$ different $\left.\mathrm{CH}_{2}+8-\mathrm{CH}+14-\mathrm{CH}\right), 1.49-$ $1.25\left(\mathrm{~m}, 5 \mathrm{H}, 4\right.$ different $\left.\mathrm{CH}_{2}\right), 1.18-1.27\left(\mathrm{~m}, 2 \mathrm{H}, \mathrm{CH}_{2}+5-\mathrm{CH}\right), 1.00-0.95\left(\mathrm{~m}, 1 \mathrm{H}, \mathrm{CH}_{2}\right), 0.93(\mathrm{~s}, 3 \mathrm{H}$, $\left.18-\mathrm{CH}_{3}\right), 0.91\left(\mathrm{~d},{ }^{3} \mathrm{~J}=6.8 \mathrm{~Hz}, 3 \mathrm{H}, \mathrm{CH}\left(\mathrm{CH}_{3}\right)_{2}\right), 0.89$ (d, 3H, $\left.\mathrm{CH}\left(\mathrm{CH}_{3}\right)_{2}\right), 0.87$ (s, 3H, 19-CH $)_{3}, 0.80-0.72$ (m, 1H, 9-CH) ppm. $-{ }^{13} \mathbf{C}$ NMR $\left(126 \mathrm{MHz}, \mathrm{CDCl}_{3}\right): \delta=209.7\left(16-C_{\mathrm{q}}-\mathrm{Ru}\right), 163.2\left(1^{\prime}-C_{\mathrm{q}} \mathrm{N}\right), 154.6(+$, $\left.3^{\prime}-C \mathrm{H}_{\mathrm{Ar}} \mathrm{N}\right), 150.7\left(17-C_{\mathrm{q}}\right), 135.9\left(+, 5^{\prime}-\mathrm{CH}_{\mathrm{Ar}}\right), 117.6\left(+, 6^{\prime}-C \mathrm{H}_{\mathrm{Ar}}\right), 116.6\left(+, 4^{\prime}-C \mathrm{H}_{\mathrm{Ar}}\right), 100.8\left(C_{\mathrm{q}, \text { cymene }}\right)$, $98.4\left(C_{\mathrm{q}, \text { cymene }}\right), 91.8\left(+, C \mathrm{H}_{\text {cymene }}\right), 85.7\left(+, C \mathrm{H}_{\text {cymene }}\right), 85.6\left(+, C \mathrm{H}_{\text {cymene }}\right), 80.3\left(+, C \mathrm{H}_{\text {cymene }}\right), 71.0(+$, 3- $\mathrm{CHOH}), 58.3(+, 14-\mathrm{CH}), 54.6(+, 9-\mathrm{CH}), 44.8(+, 5-\mathrm{CH}), 44.5\left(13-\mathrm{C}_{\mathrm{q}}\right), 44.3\left(-, \mathrm{CH}_{2}\right), 38.0\left(-, \mathrm{CH}_{2}\right)$, $36.7\left(-, \mathrm{CH}_{2}\right), 35.6\left(10-\mathrm{C}_{\mathrm{q}}\right), 35.3\left(-, \mathrm{CH}_{2}\right), 34.1(+, 8-\mathrm{CH}), 31.8\left(-, \mathrm{CH}_{2}\right), 31.3\left(-, \mathrm{CH}_{2}\right), 30.9(+$, $\left.\mathrm{CH}\left(\mathrm{CH}_{3}\right)_{2}\right), 28.7\left(-, \mathrm{CH}_{2}\right), 22.2\left(+, \mathrm{CH}\left(\mathrm{CH}_{3}\right)\right), 21.8\left(+, \mathrm{CH}\left(\mathrm{CH}_{3}\right)\right), 21.2\left(-, \mathrm{CH}_{2}\right), 18.8\left(+, \mathrm{C}_{\mathrm{q}, \text { cymene }} \mathrm{CH}_{3}\right)$, $17.4\left(+, 18-\mathrm{CH}_{3}\right), 12.2\left(+, 19-\mathrm{CH}_{3}\right)$ ppm. - IR (ATR): v = 3323 (br), 2919 (w), 2849 (w), 1663 (vw), 1595 (vw), 1502 (vw), 1465 (w), 1376 (vw), 1151 (vw), 1080 (vw), 1039 (w), 960 (vw), 921 (vw), 842 (vw), $776(\mathrm{vw}), 725$ (vw) cm ${ }^{-1}$. - MS (+FAB, 3-NBA): $m / z(\%)=618 / 619 / 620 / 621 / 622 / 623 / 624 / 625$ $[\mathrm{M}+\mathrm{H}]^{+}, 581 / 582 / 583 / 584 / 585 / 586 / 587 / 588[\mathrm{M}-\mathrm{Cl}]^{+}$. - HRMS (+FAB, $\mathrm{C}_{34} \mathrm{H}_{47} \mathrm{O}_{1} \mathrm{~N}_{1}{ }^{35} \mathrm{Cl}_{1}{ }^{102} \mathrm{Ru}_{1}$, $\left.[\mathrm{M}+\mathrm{H}]^{+}\right)$: calc. $=622.2384$; found $=622.2383$.

* NMR spectra contain traces of the other diastereomer.

[5-Bromo-2-(2-pyridinyl- $\kappa N)$ phenyl- $\kappa C]$ chlorido( $\eta^{5}$-pentamethylcyclopentadienyl)iridium(III) (9)

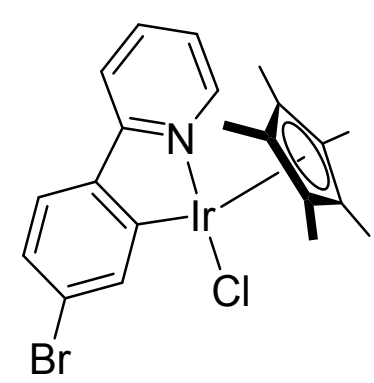

Following the general procedure, $\left[\mathrm{IrCp}^{*} \mathrm{Cl}_{2}\right]_{2} \quad(50 \mathrm{mg}, 62.8 \mu \mathrm{mol}$, 1.00 equiv.), 2-(4-bromophenyl)pyridine ( $29 \mathrm{mg}, 0.125 \mathrm{mmol}, 2.00$ equiv.) and KOAc (49 mg, $0.502 \mathrm{mmol}, 4.00$ equiv.) were dissolved in dry $\mathrm{CH}_{2} \mathrm{Cl}_{2}$ $(3.0 \mathrm{~mL})$ and stirred $24 \mathrm{~h}$ at room temperature. The suspension was concentrated and then purified via flash column chromatography on silica gel (cyclohexane/ethyl acetate, $2: 1 \rightarrow 1: 3$ ) to afford the title compound 9 as yellow solid (50 mg, $85.1 \mu \mathrm{mol}, 68 \%$ ).

$\boldsymbol{R}_{\mathbf{f}}=0.34(\mathrm{cHex} / \mathrm{EtOAc}, 2: 3) .-{ }^{1} \mathbf{H}$ NMR $\left(500 \mathrm{MHz}, \mathrm{CDCl}_{3}\right): \delta=8.67\left(\mathrm{~d}, J=5.6 \mathrm{~Hz}, 1 \mathrm{H}, 3^{\prime}-\mathrm{CH}\right), 7.91$ (d, $J=2.0 \mathrm{~Hz}, 1 \mathrm{H}, 6-\mathrm{CH}), 7.76$ (d, $\left.J=8.1 \mathrm{~Hz}, 1 \mathrm{H}, 6^{\prime}-\mathrm{CH}\right), 7.66$ (t, $\left.J=8.0 \mathrm{~Hz}, 1 \mathrm{H}, 5^{\prime}-\mathrm{CH}\right), 7.52$ (d, $J=8.2 \mathrm{~Hz}, 1 \mathrm{H}, 3-\mathrm{CH}), 7.16(\mathrm{dd}, J=8.2,2.0 \mathrm{~Hz}, 1 \mathrm{H}, 4-\mathrm{CH}), 7.13-7.06$ (m, 1H, 4'-CH), 1.67 (s, 15H, $\left.\mathrm{CH}_{3}, \mathrm{Cp}^{*}\right)$ ppm. $-{ }^{13} \mathbf{C} \mathbf{N M R}\left(126 \mathrm{MHz}, \mathrm{CDCl}_{3}\right): \delta=166.2\left(1^{\prime}-C_{\mathrm{q}}\right), 165.4\left(1-C_{\mathrm{q}}\right), 151.3\left(+, 3^{\prime}-\mathrm{CH}\right), 142.9$ $\left(2-C_{\mathrm{q}}\right), 137.8(+, 6-C \mathrm{H}), 137.1\left(+, 5^{\prime}-\mathrm{CH}\right), 125.8\left(5-C_{\mathrm{q}}-\mathrm{Br}\right), 125.0(+, 3-$ or $4-C \mathrm{H}), 124.9(+, 3-$ or $4-C \mathrm{H})$, 
$122.5\left(+, 4^{\prime}-\mathrm{CH}\right), 118.9\left(+, 6^{\prime}-\mathrm{CH}\right), 88.6\left(5 \times C_{\mathrm{q}}, \mathrm{Cp}^{*}\right), 8.76\left(+, 5 \times \mathrm{CH}_{3}, \mathrm{Cp}^{*}\right)$ ppm. - IR (ATR): $v=2961$ (w), 1731 (vw), 1601 (w), 1567 (w), 1528 (w), 1467 (w), 1416 (w), 1368 (w), 1294 (w), 1258 (m), 1160 (vw), 1070 (m), 1015 (m), 877 (w), 796 (m), 774 (m), 690 (w), 613 (w), 567 (vw), 516 (w) cm c $^{-1}$ - MS (FAB, 3-NBA): $m / z(\%)=595[\mathrm{M}]^{+}$. - HRMS (FAB, $\left.\mathrm{C}_{21} \mathrm{H}_{22} \mathrm{~N}^{79} \mathrm{Br}^{35} \mathrm{Cl}^{193} \mathrm{Ir},[\mathrm{M}]^{+}\right)$: calc. = 595.0254; found $=595.0255$.

Chlorido( $\eta^{5}$-pentamethylcyclopentadienyl)[17-(4'-pyridin-2' '-yl- $\kappa \mathrm{N}-$ phenyl-3' $\left.\kappa \mathrm{C}\right)-(5 \alpha, 3 \beta)$ androst-16enolido]iridium(III) (10)

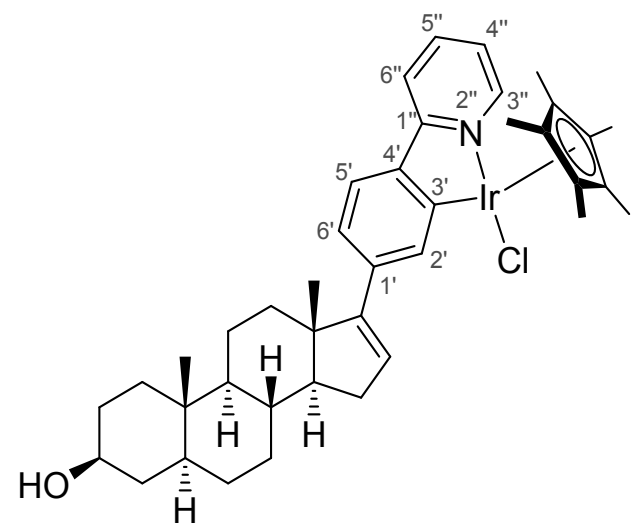

According to the general procedure $(3 \beta, 5 \alpha)-17-(4$ '-pyridin2 '-ylphenyl)androst-16-en-3-ol (5c) (25 mg, $58.5 \mu \mathrm{mol}$, 2.00 equiv.), [ $\left.\mathrm{IrCp}^{*} \mathrm{Cl}_{2}\right]_{2}(23 \mathrm{mg}, 29.2 \mu \mathrm{mol}, 1.00$ equiv.) and KOAc (9 mg, $87.7 \mu \mathrm{mol}, 3.00$ equiv.) stirred in dry $\mathrm{CH}_{2} \mathrm{Cl}_{2}(3.0 \mathrm{~mL})$ at room temperature for $20 \mathrm{~h}$. After flash column chromatography on silica gel $(c \mathrm{Hex} / \mathrm{EtOAc}$ $1: 1 \rightarrow 1: 3)$ the iridium(III) complex $\mathbf{1 0}$ was obtained as a yellow solid (16 mg, $20.5 \mu \mathrm{mol}, 70 \%$ ) in an inseparable mixture of its diastereomers $(d r=1: 1.15)$. An unambiguous assignment of the signals to the diastereomers was not possible even with 2D NMR experiments. Therefore, the signals for the diastereomeric mixture are given, same signals are stated with "and" if clearly distinguishable.

${ }^{1} \mathbf{H}$ NMR (500 MHz, $\left.\mathrm{CDCl}_{3}\right): \delta=8.66\left(\mathrm{~d}, J=5.5 \mathrm{~Hz}, 1 \mathrm{H}, \mathrm{NCH}_{\mathrm{Ar}}\right), 7.88-7.80\left(\mathrm{~m}, 1 \mathrm{H}, \mathrm{CH}_{\mathrm{Ar}}\right), 7.76(\mathrm{~d}$, $\left.J=8.1 \mathrm{~Hz}, 1 \mathrm{H}, \mathrm{C} H_{\mathrm{Ar}}\right), 7.62\left(\mathrm{td}, J=7.7,1.6 \mathrm{~Hz}, 1 \mathrm{H}, \mathrm{C} H_{\mathrm{Ar}}\right), 7.58\left(\mathrm{~d}, J=8.0 \mathrm{~Hz}, 1 \mathrm{H}, \mathrm{C} H_{\mathrm{Ar}}\right), 7.10-6.95$ $\left(\mathrm{m}, 2 \mathrm{H}, 2 \times \mathrm{CH}_{\mathrm{Ar}}\right) 5.99(\mathrm{ddd}, J=7.4,3.4,1.8 \mathrm{~Hz}, 1 \mathrm{H},=\mathrm{CH}), 3.67-3.50(\mathrm{~m}, 1 \mathrm{H}, \mathrm{CHOH}), 2.27-2.12(\mathrm{~m}$, 2H, 2 different $\left.\mathrm{CH}_{2}\right), 2.09-1.98\left(\mathrm{~m}, 1 \mathrm{H}, \mathrm{CH}_{2}\right), 1.85-1.60\left(\mathrm{~m}, 5 \mathrm{H}, 4\right.$ different $\left.\mathrm{CH}_{2}+\mathrm{CH}\right), 1.62(\mathrm{~s}, 7.5 \mathrm{H}$, $\left.5 \mathrm{CH}_{3}, \mathrm{Cp}^{*}\right), 1.61\left(\mathrm{~s}, 7.5 \mathrm{H}, 5 \mathrm{CH}_{3}, \mathrm{Cp}^{*}\right), 1.62-1.52\left(\mathrm{~m}, 2 \mathrm{H}, \mathrm{CH}_{2}+\mathrm{CH}\right), 1.53-1.24$ (m, 6H, 5 different $\left.\mathrm{CH}_{2}\right), 1.21-1.10(\mathrm{~m}, 1 \mathrm{H}, \mathrm{CH}), 1.10\left(\mathrm{~s}, 1.5 \mathrm{H}, 18-\mathrm{CH}_{3}\right), 1.09\left(\mathrm{~s}, 1.5 \mathrm{H}, 18-\mathrm{CH}_{3}\right), 1.07-0.95(\mathrm{~m}, 2 \mathrm{H}, 2$ different $\left.\mathrm{CH}_{2}\right), 0.87$ (s, 3H, 19-CH $), 0.83-0.72(\mathrm{~m}, 1 \mathrm{H}, \mathrm{CH}) \mathrm{ppm} .-{ }^{13} \mathbf{C}$ NMR $\left(126 \mathrm{MHz}, \mathrm{CDCl}_{3}\right)$ : $\delta=167.2\left(C_{\mathrm{q}}\right.$-Ir), 162.9 and 162.8 each $\left(C_{\mathrm{q}}\right), 155.8$ and 155.5 each $\left(C_{\mathrm{q}}\right) 151.2$ and 151.2 each $(+$, $\left.\mathrm{NCH}_{\mathrm{Ar}}\right), 142.8$ and 142.7 each $\left(C_{\mathrm{q}}, \mathrm{Ar}\right), 139.6$ and 139.5 each $\left(C_{\mathrm{q}, \mathrm{Ar}}\right), 136.8\left(+, C \mathrm{H}_{\mathrm{Ar}}\right), 134.0$ and 133.6 each $\left(+, C \mathrm{H}_{\mathrm{Ar}}\right), 127.4$ and 127.3 each $(+, 16-\mathrm{CH}), 123.5$ and 123.3 each $\left(+, C \mathrm{H}_{\mathrm{Ar}}\right), 121.9$ and 121.9 each $\left(+, C \mathrm{H}_{\mathrm{Ar}}\right), 121.3$ and 120.8 each $\left(+, C \mathrm{H}_{\mathrm{Ar}}\right), 118.8$ and 118.7 each $\left(+, \mathrm{CH}_{\mathrm{Ar}}\right), 88.4$ and 88.4 each $\left(+, 5 \times C_{\mathrm{q}}\right.$, $\left.\mathrm{Cp}^{*}\right), 71.3$ and 71.3 each $(+, \mathrm{CHOH}), 57.6$ and 57.6 each $(+, \mathrm{CH}), 54.7$ and 54.7 each $(+, \mathrm{CH}), 47.5$ and 47.5 each $\left(13-C_{\mathrm{q}}\right), 45.1$ and 45.1 each $(+, \mathrm{CH}), 38.2\left(-, \mathrm{CH}_{2}\right), 36.8\left(-, \mathrm{CH}_{2}\right), 35.7\left(-, \mathrm{CH}_{2}+10-\mathrm{C}_{\mathrm{q}}\right), 34.2$ and 34.1 each $(+, \mathrm{CH}), 32.0$ and 32.0 each $\left(-, \mathrm{CH}_{2}\right), 31.7$ and 31.6 each $\left(-, \mathrm{CH}_{2}\right), 31.5\left(-, \mathrm{CH}_{2}\right), 28.7$ $\left(-, \mathrm{CH}_{2}\right), 21.4$ and 21.3 each $\left(-, \mathrm{CH}_{2}\right), 17.1$ and 17.0 each $\left(+, 18-\mathrm{CH}_{3}\right), 12.4\left(+, 19-\mathrm{CH}_{3}\right), 9.01$ and 8.99 each $\left(+, 5 \times \mathrm{CH}_{3}, \mathrm{Cp}^{*}\right)$ ppm. - IR (ATR): v = 3408 (br), 2914 (w), 2848 (vw), 1604 (vw), 1579 (vw), 
1560 (vw), 1470 (vw), 1376 (vw), 1316 (vw), 1265 (vw), 1157 (vw), 1030 (vw), 885 (vw), 810 (vw), 773 (w), 727 (vw), 613 (vw), 500 (vw), 434 (vw) cm c $^{-1}$ - MS (FAB, 3-NBA): $m / z(\%)=793 / 792 / 791 /$ 790/789/788/787 [M] $]^{+}, 756 / 755 / 754 / 753 / 752 / 751 / 750[\mathrm{M}-\mathrm{Cl}]^{+}, 363\left[\mathrm{IrCp}^{*} \mathrm{Cl}\right]^{+} .-$HRMS (FAB, $\mathrm{C}_{40} \mathrm{H}_{51} \mathrm{ON}^{315} \mathrm{Cl}^{193} \mathrm{Ir},[\mathrm{M}]^{+}$): calc. $=789.3289$; found $=789.3291$.

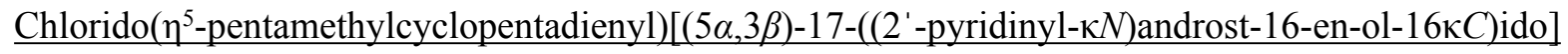
iridium(III) (11)

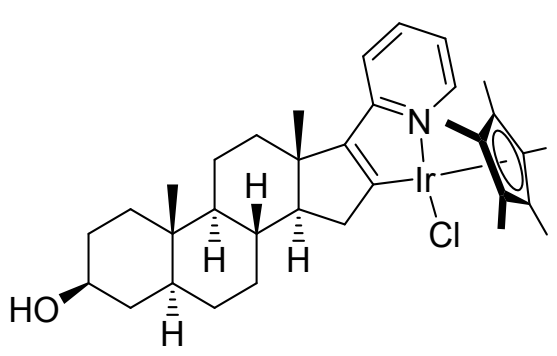

According to the general procedure $(3 \beta, 5 \alpha)-17-\left(2^{\prime}\right.$-pyridinyl)androst-16-en-3-ol (5a) $\quad(77 \mathrm{mg}, \quad 218 \mu \mathrm{mol}, \quad 2.00$ equiv.), [IrCp*Cl $\left.]_{2}\right]_{2}(87 \mathrm{mg}, 109 \mu \mathrm{mol}, 1.00$ equiv.) and KOAc (32 mg, $327 \mu \mathrm{mol}, 3.00$ equiv.) in dry $\mathrm{CH}_{2} \mathrm{Cl}_{2}(5.0 \mathrm{~mL})$ were stirred at room temperature for $26 \mathrm{~h}$. After flash column chromatography on silica gel $(c \mathrm{Hex} / \mathrm{EtOAc}, 2: 1 \rightarrow 1: 1)$ the iridium(III) complex 10 was obtained as a yellow solid ( $76 \mathrm{mg}, 0.107 \mathrm{mmol}, 48 \%$ ) as a mixture of its diastereomers $(d r=1: 1.15)$. An unambiguous assignment of the signals to the diastereomers was not possible even with 2D NMR experiments. Therefore, the signals for the diastereomeric mixture are given, same signals are stated with "and" if clearly distinguishable.

${ }^{1} \mathrm{H}$ NMR (500 MHz, $\left.\mathrm{CDCl}_{3}\right): \delta=8.51(\mathrm{dd}, J=5.8,1.5 \mathrm{~Hz}, 0.64 \mathrm{H}, 3-\mathrm{C} H \mathrm{~N}$, major) and $8.44(\mathrm{dd}, J=5.8$, $1.5 \mathrm{~Hz}, 0.36 \mathrm{H}, 3-\mathrm{CHN}$ ), 7.44-7.32 (m, 1H, 5-CH, both), 7.22-7.15 (m, 1H, 6-CH, both), 6.70 (td, $J=6.5,5.8,1.3 \mathrm{~Hz}, 1 \mathrm{H}, 4-\mathrm{CH}$, both) 3.60 (tt, $J=10.6,4.7 \mathrm{~Hz}, 1 \mathrm{H}, \mathrm{CHOH}$, both) 2.86 (dd, $J=16.3$, $6.1 \mathrm{~Hz}, 0.64 \mathrm{H}, 15-\mathrm{CH}_{2}{ }^{\mathrm{a}}$, major) and $2.73\left(\mathrm{dd}, J=16.2,6.4 \mathrm{~Hz}, 0.36 \mathrm{H}, 15-\mathrm{CH}_{2}{ }^{\mathrm{a}}\right.$, minor), 2.59 (dd, $J=16.2,11.3 \mathrm{~Hz}, 0.36 \mathrm{H}, 15-\mathrm{CH}_{2}{ }^{\mathrm{b}}$, minor), $2.28-2.17$ (m, $2 \mathrm{H}, \mathrm{CH}_{2}$, both $+15-\mathrm{CH}_{2}{ }^{\mathrm{b}}$, major), 1.87-1.74 (m, $2 \mathrm{H}, 2$ different $\mathrm{CH}_{2}$, both), 1.71 (s, $10 \mathrm{H}, 5 \times \mathrm{CH}_{3}\left(\mathrm{Cp}^{*}\right)$, major), 1.68 (s, $5 \mathrm{H}, 5 \times \mathrm{CH}_{3}\left(\mathrm{Cp}^{*}\right)$, minor), 1.70-1.43 (m, 7H, different $\mathrm{CH}_{2}+\mathrm{CH}+\mathrm{CH}$, both), 1.35-1.27 (m, 3.36H, 2 different $\mathrm{CH}_{2}$, both $+\mathrm{CH}$, minor), 1.19-0.98 (m, 4H, 2 different $\mathrm{CH}_{2}$, both $+\mathrm{CH}$, major), 0.97 (s, $1 \mathrm{H}, 18-\mathrm{CH}_{3}$, minor), 0.87 and 0.86 (two s, $5 \mathrm{H}, 18-\mathrm{CH}_{3}$, major $+19-\mathrm{CH}_{3}$, major $+19-\mathrm{CH}_{3}$, minor), $0.83-0.71(\mathrm{~m}, 1 \mathrm{H}, \mathrm{CH}$, both) ppm. $-{ }^{13} \mathrm{C}$ NMR (126 MHz, $\left.\mathrm{CDCl}_{3}\right): \delta=187.9$ and 187.2 each $\left(16-C_{\mathrm{q}}-\mathrm{Ir}\right), 165.1$ and 164.9 each $\left(1-C_{\mathrm{q}} \mathrm{N}\right)$, 153.2 and 151.9 each $\left(17-C_{\mathrm{q}}\right), 151.6$ and $151.5\left(+, C \mathrm{H}_{\mathrm{pyr}}\right), 136.7$ and 136.5 each $\left(+, C \mathrm{H}_{\mathrm{pyr}}\right), 117.8$ and 117.6 each $\left(+, C \mathrm{H}_{\mathrm{pyr}}\right), 117.4$ and 116.9 each $\left(+, C \mathrm{H}_{\mathrm{pyr}}\right), 88.5$ and 88.5 each $\left(\mathrm{C}_{\mathrm{q}, \mathrm{Ar}}, 5 \times \mathrm{Cp}^{*}\right), 71.4$ and 71.2 each $(+, \mathrm{CHOH}), 59.1$ and 58.7 each $(+, \mathrm{CH}), 55.3$ and 54.7 each $(+, \mathrm{CH}), 45.5$ and $45.3\left(13-C_{\mathrm{q}}\right), 45.2$ and $45.0\left(-, \mathrm{CH}_{2}\right), 39.3$ and 39.1 each $\left(-, \mathrm{CH}_{2}\right), 38.3$ and 38.2 each $\left(-, \mathrm{CH}_{2}\right), 37.0$ and 36.8 each (-, $\left.\mathrm{CH}_{2}\right), 36.3$ and 35.3 each $\left(-, \mathrm{CH}_{2}\right), 35.8$ and 35.7 each (10- $\left.C_{\mathrm{q}}\right), 34.4$ and 34.1 each $(+, \mathrm{CH}), 32.3$ and 31.9 each $\left(-, \mathrm{CH}_{2}\right), 31.6$ and 31.5 each $\left(-, \mathrm{CH}_{2}\right), 28.8\left(-, \mathrm{CH}_{2}\right), 21.6$ and 21.4 each $\left(-, \mathrm{CH}_{2}\right), 18.4$ and 16.5 each $\left(+, 18-\mathrm{CH}_{3}\right), 12.4$ and 12.4 each $\left(+, 19-\mathrm{CH}_{3}\right), 9.2$ and 9.1 each $\left(+, 5 \times \mathrm{CH}_{3}, \mathrm{Cp} *\right)$ ppm. $-\mathbf{I R}$ (ATR): v = 3410 (br), 2914 (w), $2848(\mathrm{vw}), 1597(\mathrm{vw}), 1500(\mathrm{vw}), 1466(\mathrm{w}), 1375(\mathrm{vw}), 1242(\mathrm{vw})$, $1080(\mathrm{vw}), 1026$ (w), 961 (vw), 776 (w), 726 (m), 643 (vw) $\mathrm{cm}^{-1}$. - MS (FAB, 3-NBA): m/z 
$(\%)=717 / 716 / 715 / 714 / 713 / 712 / 711 \quad[\mathrm{M}]^{+}, 680 / 679 / 678 / 677 / 676 / 675 / 674 \quad[\mathrm{M}-\mathrm{Cl}]^{+}, 663 / 662 / 661 /$ 660/659/658 $\left[\mathrm{M}-\mathrm{Cl}-\mathrm{OH} / \mathrm{H}_{2} \mathrm{O}\right]^{+}, \quad 363 \quad\left[\mathrm{IrCp}^{*} \mathrm{Cl}\right]^{+} .-$HRMS $\quad\left(\mathrm{FAB}, \mathrm{C}_{34} \mathrm{H}_{47} \mathrm{ON}^{35} \mathrm{Cl}^{193} \mathrm{Ir}, \quad[\mathrm{M}]^{+}\right)$: calc. $=713.2976$; found $=713.2974$.

Dichlorido( $\eta^{6}$-para-cymene) $\left[(5 \alpha, 3 \beta)-17-\left(\left(3^{\prime}\right.\right.\right.$-pyridinyl- $\left.\kappa \mathrm{N}\right)$-androst-16-en-3-ol)ido]ruthenium(II) (12)

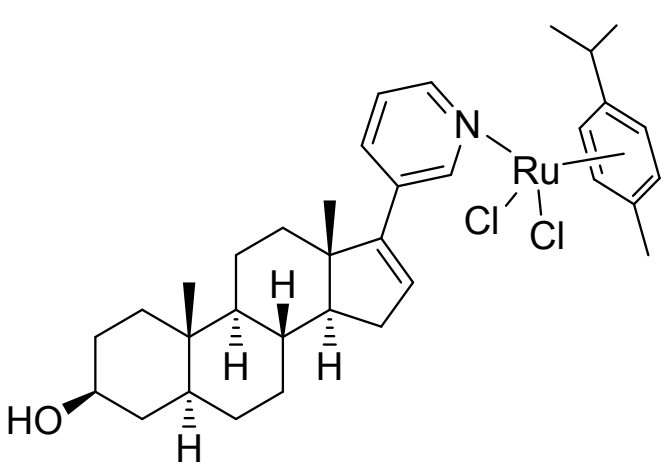

Under argon atmosphere, (3 $\beta, 5 \alpha)-17-\left(3^{\prime}\right.$-pyridinyl)androst-16-en-3-ol (5b) (22 mg, $62.6 \mu \mathrm{mol}, 2.00$ equiv.) and $\left[\mathrm{Ru}(p \text {-cymene }) \mathrm{Cl}_{2}\right]_{2}(19 \mathrm{mg}, 31.1 \mu \mathrm{mol}, 1.00$ equiv. $)$ were in dry $\mathrm{CH}_{2} \mathrm{Cl}_{2}(1.5 \mathrm{~mL})$. The solution was stirred $24 \mathrm{~h}$ at room temperature then, a mixture of $n$-hexane and ethyl acetate $(10 \mathrm{~mL}, 4: 1)$ was added. The precipitate was washed with $n$-hexane (ca. $30 \mathrm{~mL}$ ) and the title compound 12 was obtained as an orange solid (14 mg, $21.7 \mu \mathrm{mol}, 70 \%)$.

${ }^{1} \mathbf{H}$ NMR $\left(500 \mathrm{MHz}, \mathrm{CDCl}_{3}\right): \delta=9.02\left(\mathrm{~d}, J=2.0 \mathrm{~Hz}, 1 \mathrm{H}, 2^{\prime}-\mathrm{CH}\right), 8.84\left(\mathrm{~d}, J=5.3 \mathrm{~Hz}, 1 \mathrm{H}, 4{ }^{\prime}-\mathrm{CH}\right), 7.67$ (dt, $\left.J=8.0,1.7 \mathrm{~Hz}, 1 \mathrm{H}, 6^{\prime}-\mathrm{CH}\right), 7.21\left(\mathrm{dd}, J=7.9,5.6 \mathrm{~Hz}, 1 \mathrm{H}, 5^{\prime}-\mathrm{CH}\right), 6.05(\mathrm{~d}, J=1.6 \mathrm{~Hz}, 1 \mathrm{H},=\mathrm{C} H)$, $5.50-5.37\left(\mathrm{~m}, 2 \mathrm{H}, 2 \times \mathrm{C} H_{\text {cymene }}\right), 5.21\left(\mathrm{~d}, J=5.9 \mathrm{~Hz}, 2 \mathrm{H}, 2 \times \mathrm{C} H_{\text {cymene }}\right), 3.60(\mathrm{tt}, J=10.5,4.5 \mathrm{~Hz}, 1 \mathrm{H}$, $\mathrm{C} H \mathrm{OH}), 2.23\left(\mathrm{ddd}, J=6.1,6.5,3.4 \mathrm{~Hz}, 1 \mathrm{H}, 15-\mathrm{CH}_{2}^{\mathrm{a}}\right), 2.12\left(\mathrm{~s}, 3 \mathrm{H}, \mathrm{C}_{\mathrm{q}, \text { cymene }} \mathrm{CH}_{3}\right), 2.08-1.92(\mathrm{~m}, 2 \mathrm{H}$, 15- $\left.\mathrm{CH}_{2}{ }^{\mathrm{b}}+\mathrm{CH}_{2}\right), 1.83-1.78\left(\mathrm{~m}, 1 \mathrm{H}, \mathrm{CH}_{2}\right), 1.76-1.50\left(\mathrm{~m}, 6 \mathrm{H}, 6 \mathrm{H}, 4\right.$ different $\left.\mathrm{CH}_{2}+\mathrm{CH}+\mathrm{CH}\right), 1.49-$ $1.39\left(\mathrm{~m}, 3 \mathrm{H}, 3\right.$ different $\left.\mathrm{CH}_{2}\right), 1.31\left(\mathrm{~s}, 3 \mathrm{H}, \mathrm{CHCH}_{3}\right), 1.30\left(\mathrm{~s}, 3 \mathrm{H}, \mathrm{CHCH}_{3}\right), 1.30-1.23$ (m, 4H, 2 different $\left.\mathrm{CH}_{2}+\mathrm{CH}\left(\mathrm{CH}_{3}\right)_{2}\right), 1.19-1.09(\mathrm{~m}, 1 \mathrm{H}, \mathrm{CH}), 1.02-0.95\left(\mathrm{~m}, 2 \mathrm{H}, 2\right.$ different $\left.\mathrm{CH}_{2}\right), 1.00\left(\mathrm{~s}, 3 \mathrm{H}, 18-\mathrm{CH}_{3}\right)$, $0.85\left(\mathrm{~s}, 3 \mathrm{H}, 19-\mathrm{CH}_{3}\right), 0.80-0.70(\mathrm{~m}, 1 \mathrm{H}, \mathrm{CH})$ ppm. $-{ }^{13} \mathbf{C}$ NMR $\left(126 \mathrm{MHz}, \mathrm{CDCl}_{3}\right): \delta=152.6(+$, $\left.2^{\prime}, 4^{\prime}-C H\right), 150.2\left(1^{\prime}-C_{\mathrm{q}}\right), 134.8\left(+, 6^{\prime}-C H\right), 133.9\left(17-C_{\mathrm{q}}\right), 131.2(+, 16-C H), 123.7\left(+, 5^{\prime}-C H\right), 103.5$ $\left(C_{\text {q,cymene }}\right), 96.8\left(C_{\text {q,cymene }}\right), 82.4\left(+, C \mathrm{H}_{\text {cymene }}\right), 82.3\left(+, 2 \times C \mathrm{H}_{\text {cymene }}\right), 82.3\left(+, C \mathrm{H}_{\text {cymene }}\right), 71.1(+, 3-C \mathrm{HOH})$, $57.3(+, C H), 54.4(+, C H), 47.4\left(13-C_{\mathrm{q}}\right), 44.9(+, C H), 38.0\left(-, \mathrm{CH}_{2}\right), 36.7\left(-, \mathrm{CH}_{2}\right), 35.5\left(10-C_{\mathrm{q}}\right), 35.1$ $\left(-, \mathrm{CH}_{2}\right), 33.8(+, \mathrm{CH}), 31.8\left(-, \mathrm{CH}_{2}\right), 31.7\left(-, \mathrm{CH}_{2}\right), 31.4\left(-, \mathrm{CH}_{2}\right), 30.5\left(+, \mathrm{CH}\left(\mathrm{CH}_{3}\right)_{2}\right), 28.5\left(-, \mathrm{CH}_{2}\right)$, $22.2\left(+, \mathrm{CHCH}_{3}\right), 22.1\left(+, \mathrm{CHCH}_{3}\right), 21.1\left(-, \mathrm{CH}_{2}\right), 18.1\left(+, \mathrm{C}_{\mathrm{q}, \text { cymene }} \mathrm{CH}_{3}\right), 16.7\left(+, 18-\mathrm{CH}_{3}\right), 12.2(+$, 19- $\mathrm{CH}_{3}$ ) ppm. - IR (ATR): v = 3416 (br), 2919 (w), 2848 (w), 1601 (vw), 1443 (vw), 1413 (vw), 1376 (vw), 1262 (vw), 1079 (vw), 1039 (w), 923 (vw), 867 (vw), 800 (w), 730 (vw), 698 (vw), 667 (vw) $\mathrm{cm}^{-1}$. - MS (FAB, 3-NBA): $m / z(\%)=662 / 661 / 660 / 659 / 658 / 657 / 656 / 655[\mathrm{M}]^{+}, 626 / 625 / 624 / 623 /$ 622/621/620/619 [M-Cl] $]^{+}$- HRMS (FAB, $\left.\mathrm{C}_{34} \mathrm{H}_{47} \mathrm{ON}^{35} \mathrm{Cl}^{102} \mathrm{Ru}, \quad[\mathrm{M}]^{+}\right)$: calc. =657.2078; found $=657.2079$. 
Dichlorido $\left(\eta^{5}\right.$-pentamethylcyclopentadienyl $)\left[(5 \alpha, 3 \beta)-17-\left(\left(3^{\prime}\right.\right.\right.$-pyridinyl- $\left.\kappa \mathrm{N}\right)$ androst-16-en-3-ol)ido]$\underline{\text { iridium(III) (13) }}$

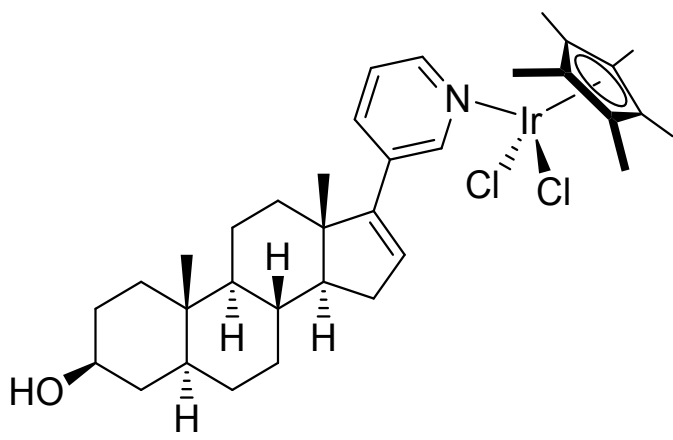

Under argon atmosphere, $(3 \beta, 5 \alpha)-17-\left(3^{\prime}-\right.$ pyridinyl)androst-16-en-3-ol (5b) $(32 \mathrm{mg}, 91.0 \mu \mathrm{mol}$, 2.00 equiv.) and $\left[\mathrm{IrCp}^{*} \mathrm{Cl}_{2}\right]_{2} \quad(36 \mathrm{mg}, 45.5 \mu \mathrm{mol}$, 1.00 equiv.) were in dry $\mathrm{CH}_{2} \mathrm{Cl}_{2}(3.0 \mathrm{~mL})$. The solution was stirred $24 \mathrm{~h}$ at room temperature. A mixture of $n$ hexane and ethyl acetate $(10 \mathrm{~mL}, 4: 1)$ was added. The precipitate was washed with $n$-hexane (ca. $30 \mathrm{~mL}$ ) and the title compound 13 was obtained as a yellow solid (52 mg, $68.8 \mu \mathrm{mol}, 76 \%)$.

${ }^{1} \mathbf{H}$ NMR (500 MHz, $\left.\mathrm{CDCl}_{3}\right): \delta=8.99\left(\mathrm{~d}, J=1.9 \mathrm{~Hz}, 1 \mathrm{H}, 2^{\prime}-\mathrm{CH}_{\mathrm{pyr}}\right), 8.77$ (d, $\left.J=5.6 \mathrm{~Hz}, 1 \mathrm{H}, 4^{\prime}-\mathrm{CH}_{\mathrm{pyr}}\right)$, $7.67\left(\mathrm{~d}, J=7.8 \mathrm{~Hz}, 1 \mathrm{H}, 5^{\prime}-\mathrm{CH}_{\mathrm{pyr}}\right), 7.26-7.15\left(\mathrm{~m}, 1 \mathrm{H}, 6^{\prime}-\mathrm{CH}_{\mathrm{pyr}}\right), 6.14-6.06(\mathrm{~m}, 1 \mathrm{H}, 16-\mathrm{CH}), 3.67-$ $3.49(\mathrm{~m}, 1 \mathrm{H}, 3-\mathrm{CHOH}), 2.24\left(\mathrm{ddd}, J=16.1,6.4,3.3 \mathrm{~Hz}, 1 \mathrm{H}, 15-\mathrm{CH}_{2}{ }^{\mathrm{a}}\right), 2.10-1.97\left(\mathrm{~m}, 2 \mathrm{H}, 15-\mathrm{CH}_{2}{ }^{\mathrm{b}}+\right.$ $\left.\mathrm{CH}_{2}\right), 1.85-1.77\left(\mathrm{~m}, 1 \mathrm{H}, \mathrm{CH}_{2}\right), 1.74-1.55\left(\mathrm{~m}, 6 \mathrm{H}, 4\right.$ different $\left.\mathrm{CH}_{2}+\mathrm{CH}+\mathrm{CH}\right), 15.3\left(\mathrm{~m}, 15 \mathrm{H}, 5 \times \mathrm{CH}_{3}\right.$, $\left.\mathrm{Cp}^{*}\right)$ 1.46-1.20 (m, 6H, 5 different $\left.\mathrm{CH}_{2}\right), 1.19-1.07(\mathrm{~m}, 1 \mathrm{H}, \mathrm{CH}), 1.07-0.98\left(\mathrm{~m}, 2 \mathrm{H}, 2\right.$ different $\left.\mathrm{CH}_{2}\right)$, $1.01\left(\mathrm{~s}, 3 \mathrm{H}, 18-\mathrm{CH}_{3}\right), 0.85\left(\mathrm{~s}, 3 \mathrm{H}, 19-\mathrm{CH}_{3}\right), 0.81-0.71(\mathrm{~m}, 1 \mathrm{H}, \mathrm{CH}) \mathrm{ppm} .-{ }^{13} \mathbf{C}$ NMR $(126 \mathrm{MHz}$, $\left.\mathrm{CDCl}_{3}\right): \delta=151.2\left(+, 2^{\prime}-\right.$ or $\left.4^{\prime}-\mathrm{CH}_{\mathrm{pyr}}\right), 151.0\left(+, 2^{\prime}-\right.$ or $\left.4^{\prime}-\mathrm{CH}_{\mathrm{pyr}}\right), 150.1\left(17-C_{\mathrm{q}}\right), 135.0\left(+, 5^{\prime}-\mathrm{CH}_{\mathrm{pyr}}\right)$, $134.8\left(1^{\prime}-C_{\mathrm{q}}\right), 131.3(+, 16-\mathrm{CH}), 124.6\left(+, 6^{\prime}-\mathrm{CH}_{\mathrm{pyr}}\right), 85.5\left(5 \times C_{\mathrm{q}}, \mathrm{Cp}^{*}\right), 71.1(+, \mathrm{CHOH}), 57.2(+, \mathrm{CH})$, $54.4(+, \mathrm{CH}), 47.4\left(13-\mathrm{C}_{\mathrm{q}}\right), 44.9(+, \mathrm{CH}), 38.0\left(-, \mathrm{CH}_{2}\right), 36.7\left(-, \mathrm{CH}_{2}\right), 35.6\left(10-\mathrm{C}_{\mathrm{q}}\right), 35.2\left(-, \mathrm{CH}_{2}\right), 33.8$ (+, $\mathrm{CH}), 31.8\left(-, \mathrm{CH}_{2}\right), 31.7\left(-, \mathrm{CH}_{2}\right), 31.4\left(-, \mathrm{CH}_{2}\right), 28.5\left(-, \mathrm{CH}_{2}\right), 21.1\left(-, \mathrm{CH}_{2}\right), 16.7\left(+, 18-\mathrm{CH}_{3}\right)$, $12.2\left(+, 19-\mathrm{CH}_{3}\right), 8.4\left(+, 5 \times \mathrm{CH}_{3}, \mathrm{Cp}^{*}\right)$ ppm. - IR (ATR): v = $3402(\mathrm{br}), 2925(\mathrm{w}), 2852(\mathrm{w}), 1599(\mathrm{vw})$, 1445 (w), 1422 (vw), 1375 (w), 1291 (vw), 1224 (vw), 1193 (vw), 1117 (vw), 1082 (w), 1049 (w), 1029 (w), 922 (vw), 841 (vw), 804 (w), 732 (w), 703 (w), 611 (vw), 538 (vw) cm-1. - MS (FAB, 3-NBA): $m / z \quad(\%)=752 / 751 / 750 / 749 / 748 / 747 / 746 \quad[\mathrm{M}]^{+}, \quad 718 / 717 / 716 / 715 / 714 / 713 / 712 \quad[\mathrm{M}-\mathrm{Cl}]^{+}, \quad 398$ $\left[\mathrm{IrCp}^{*} \mathrm{Cl}_{2}\right]^{+}, \quad 363 \quad\left[\mathrm{IrCp}^{*} \mathrm{Cl}\right]^{+} . \quad-$ HRMS $\quad\left(\mathrm{FAB}, \mathrm{C}_{34} \mathrm{H}_{48} \mathrm{ON}^{35} \mathrm{Cl}_{2}{ }^{193} \mathrm{Ir}, \quad[\mathrm{M}]^{+}\right)$: calc. $=749.2742$; found $=749.2741$. 


\subsection{NMR spectra of the synthesized compounds}

\section{$\underline{(3 \beta, 5 \alpha)-17-B r o m o a n d r o s t-16-e n-3-o l ~(4 a)})^{2}$}

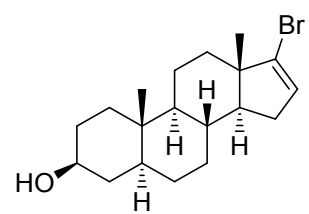

${ }^{1} \mathrm{H}$ NMR: $400 \mathrm{MHz}, \mathrm{CDCl}_{3}$
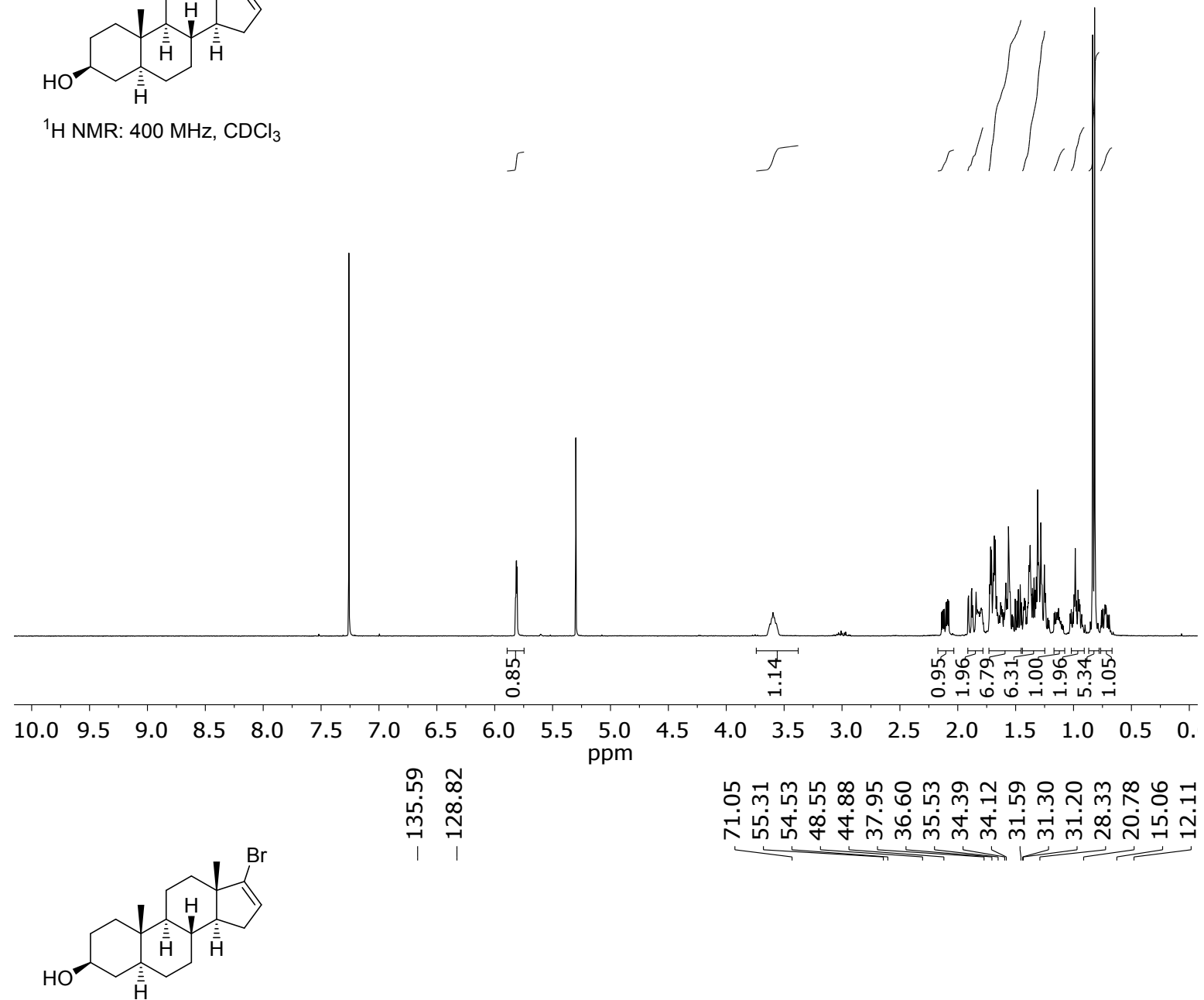

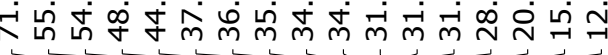

${ }^{13} \mathrm{C}$ NMR: $101 \mathrm{MHz}, \mathrm{CDCl}_{3}$

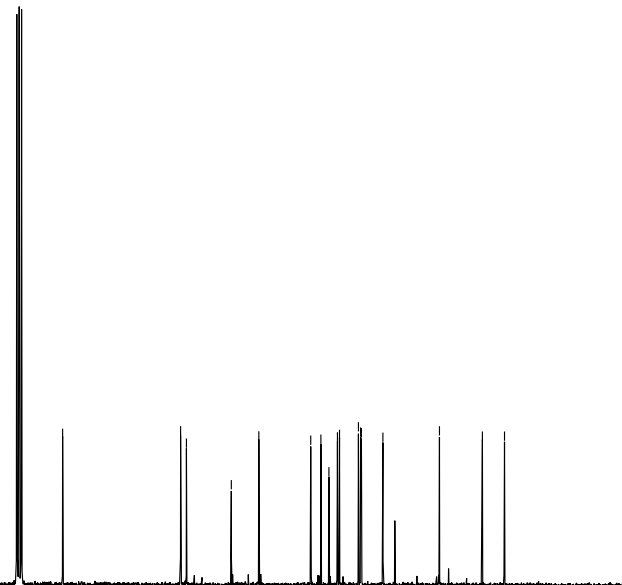

$\begin{array}{lllllllllllllllllllll}200 & 190 & 180 & 170 & 160 & 150 & 140 & 130 & 120 & 110 & \begin{array}{c}100 \\ \mathrm{ppm}\end{array} & 90 & 80 & 70 & 60 & 50 & 40 & 30 & 20 & 10 & 0\end{array}$

NMR spectra contain traces of dichloromethane. 


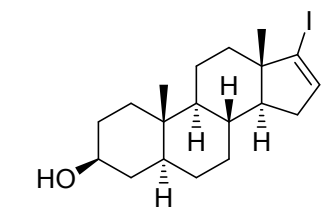

${ }^{1} \mathrm{H}$ NMR: $400 \mathrm{MHz}, \mathrm{CDCl}_{3}$
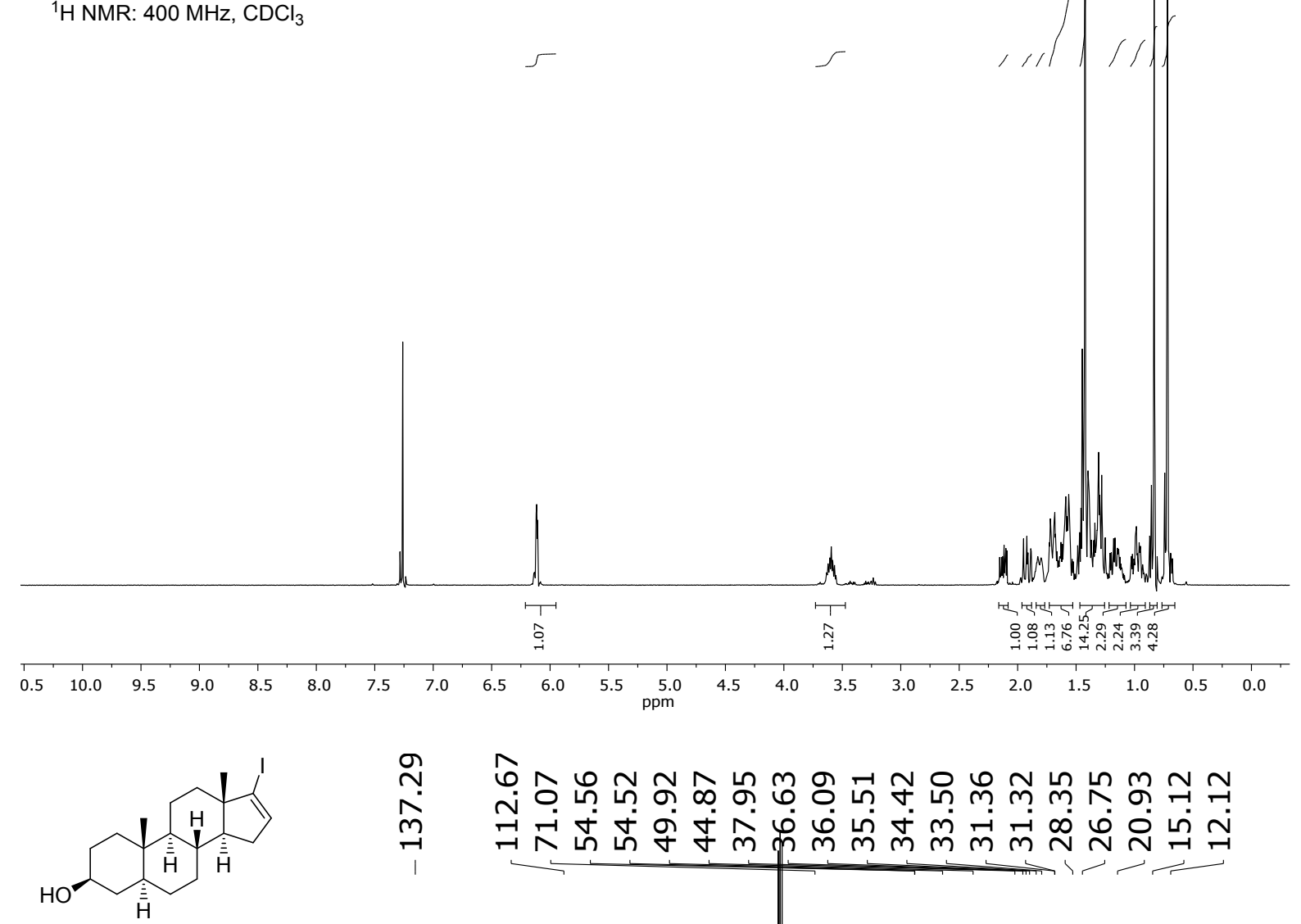

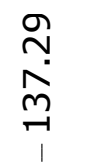

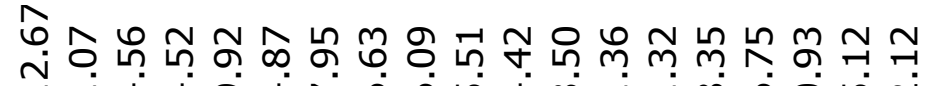
m ت $\quad$ ت

${ }^{13} \mathrm{C}$ NMR: $101 \mathrm{MHz}, \mathrm{CDCl}_{3}$

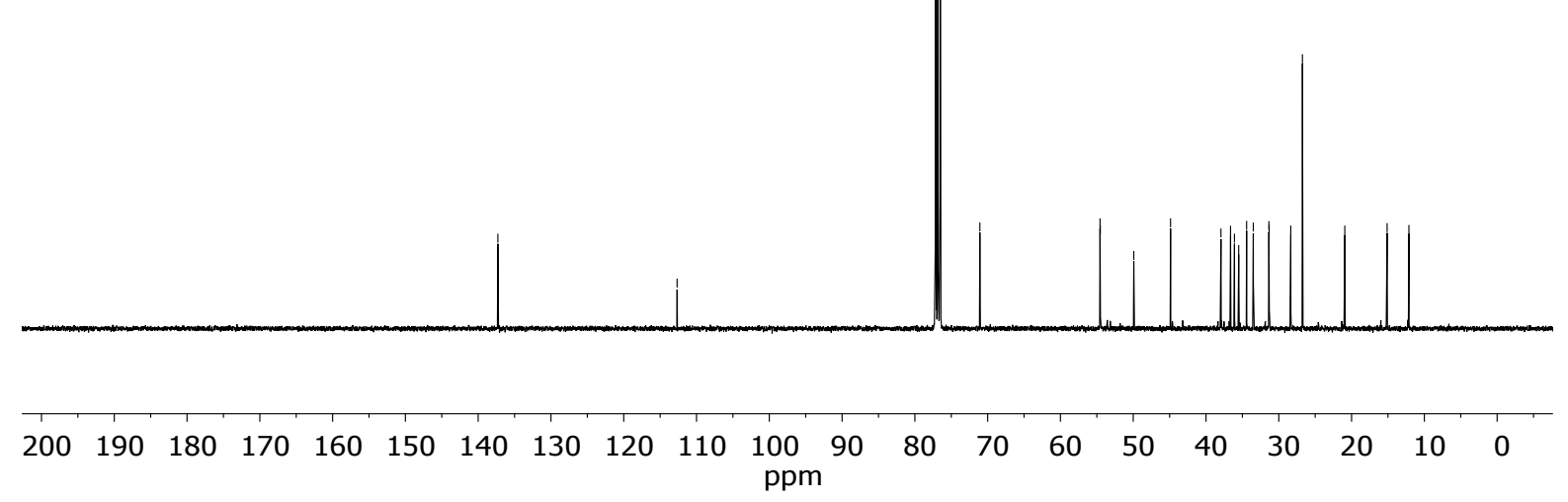

NMR spectra contain traces of cyclohexane. 


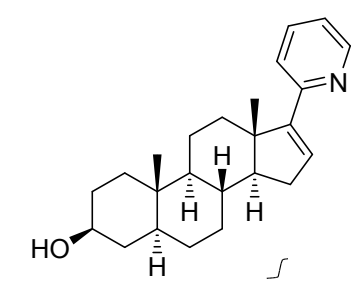

${ }^{1} \mathrm{H}$ NMR: $400 \mathrm{MHz}, \mathrm{CDCl}_{3}$
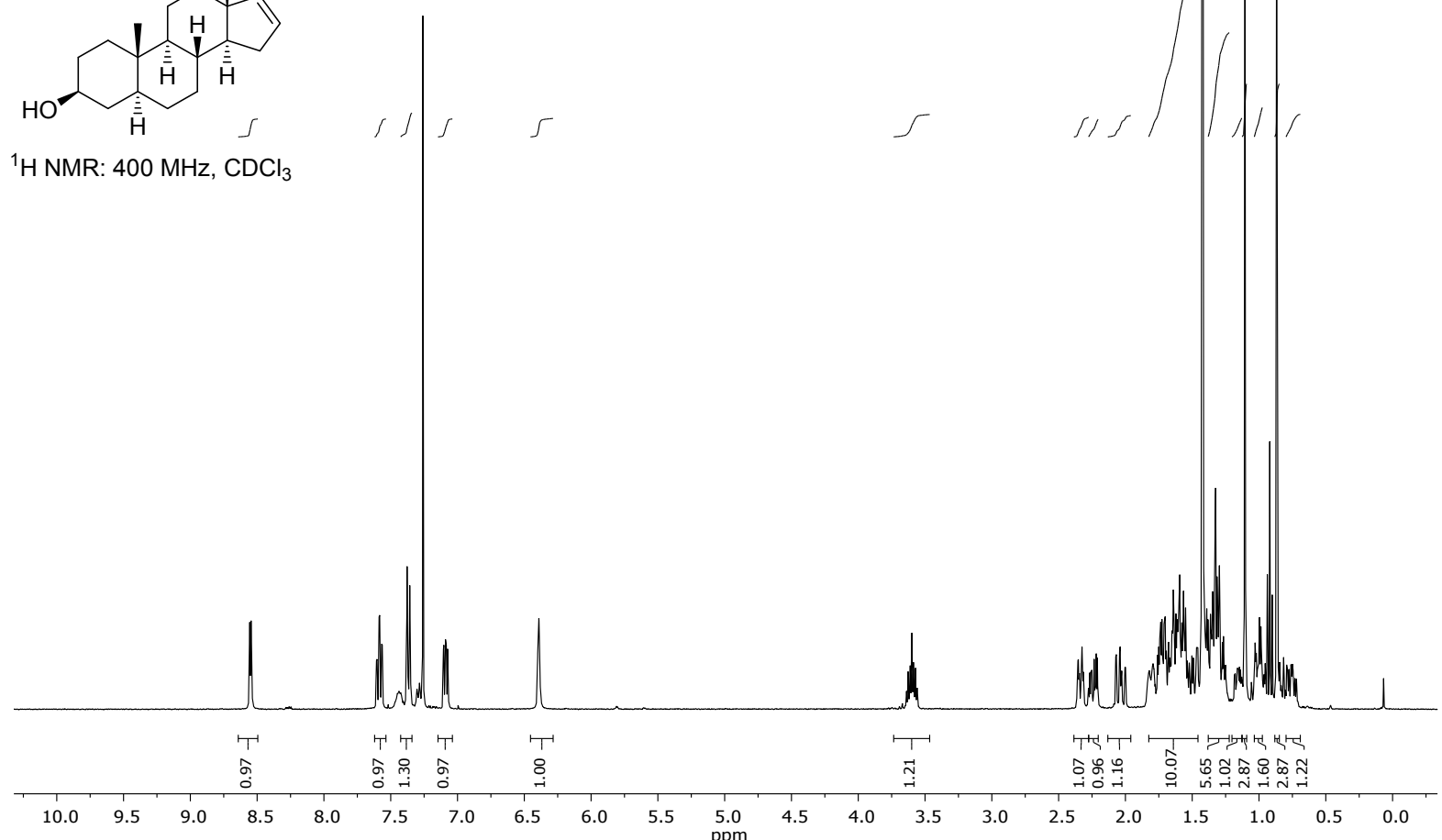

กำ $\underset{\sim}{m} \infty$

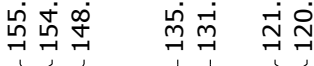

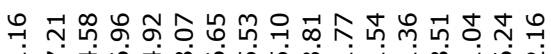

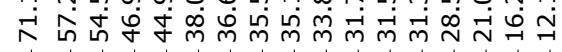

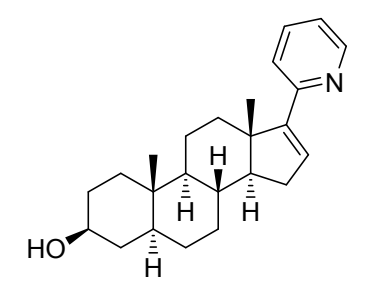

${ }^{13} \mathrm{C}-\mathrm{NMR}: 101 \mathrm{MHz}, \mathrm{CDCl}_{3}$

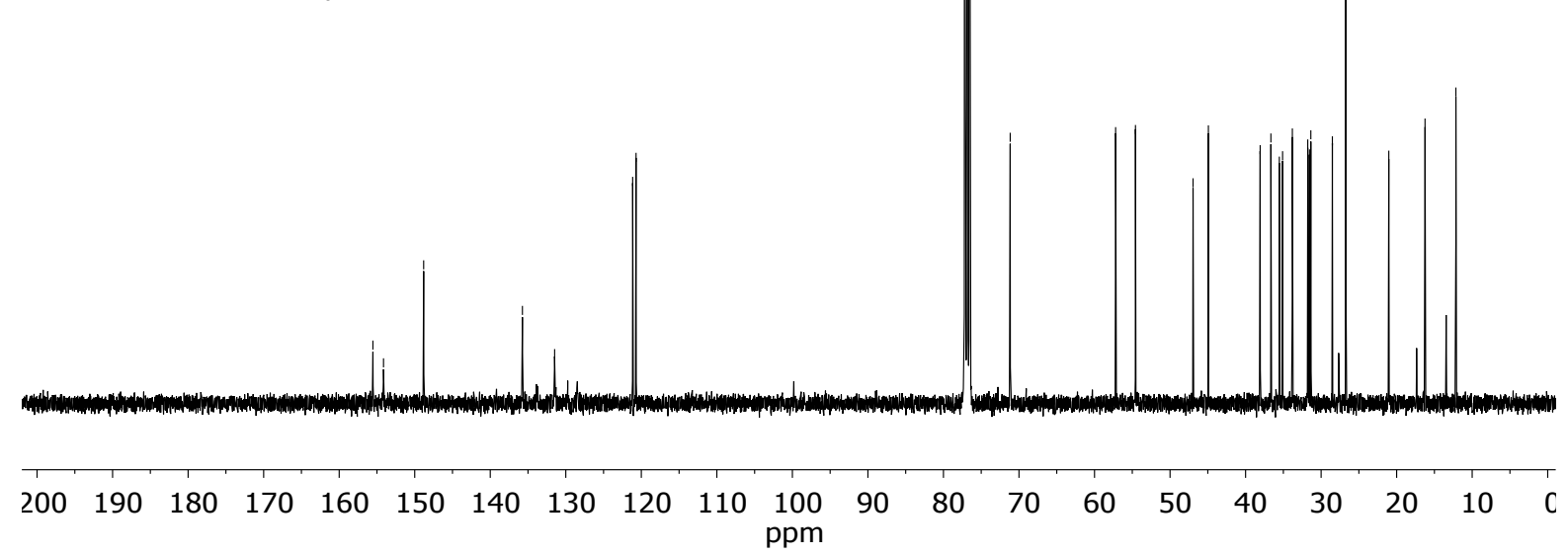




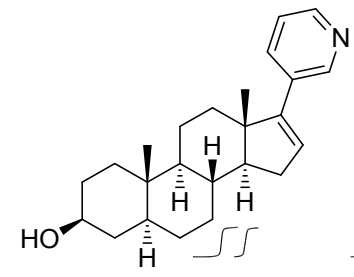

${ }^{1} \mathrm{H}$ NMR: $400 \mathrm{MHz}, \mathrm{CDCl}_{3}$
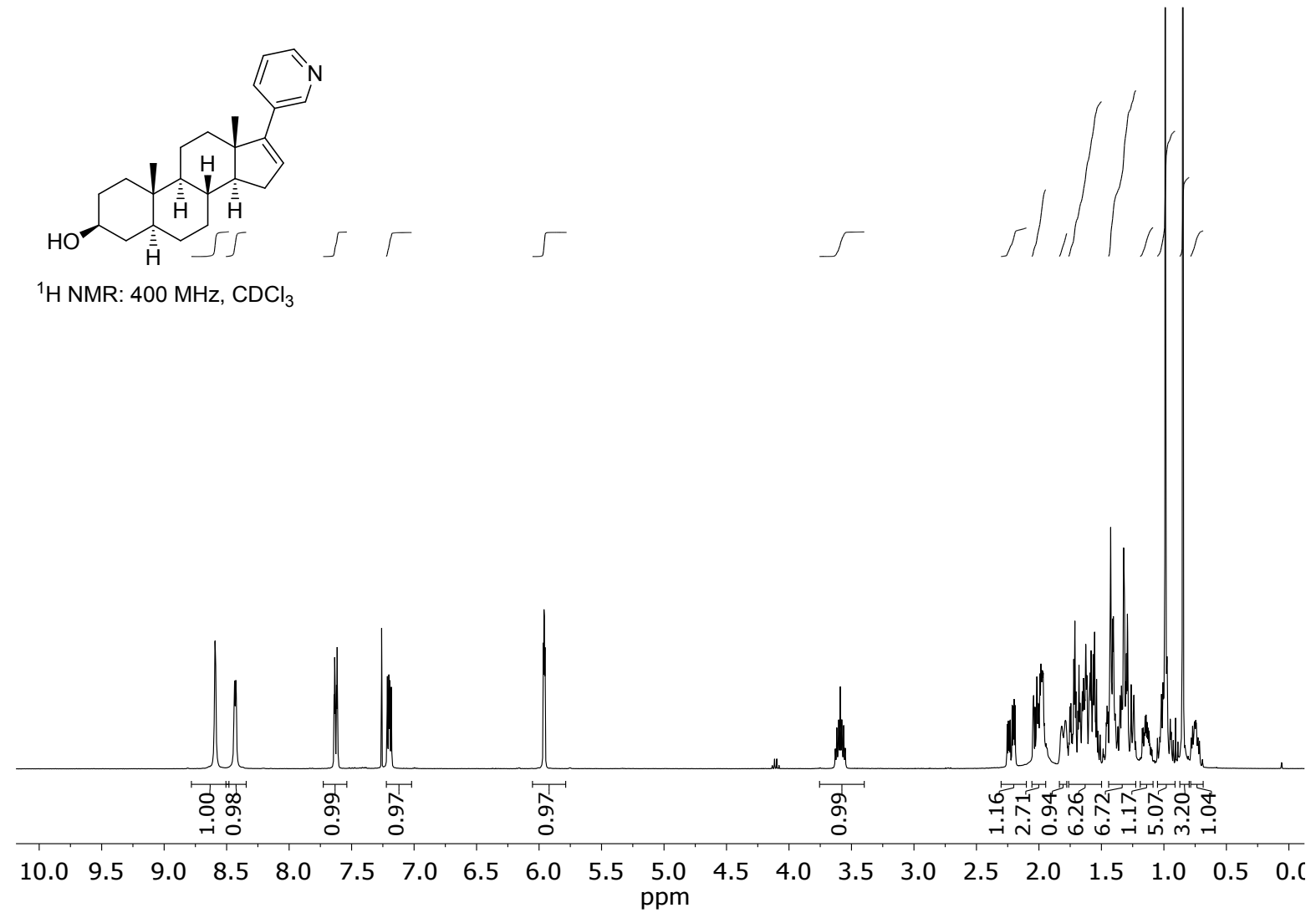

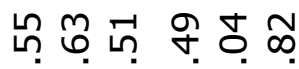

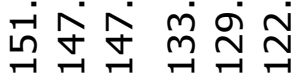

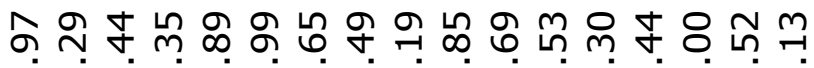

군,

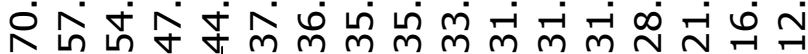

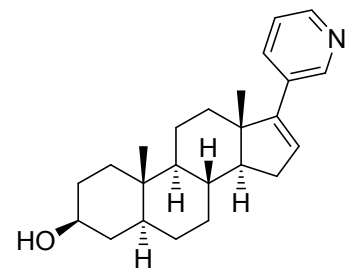

${ }^{13} \mathrm{C}$ NMR: $101 \mathrm{MHz}, \mathrm{CDCl}_{3}$

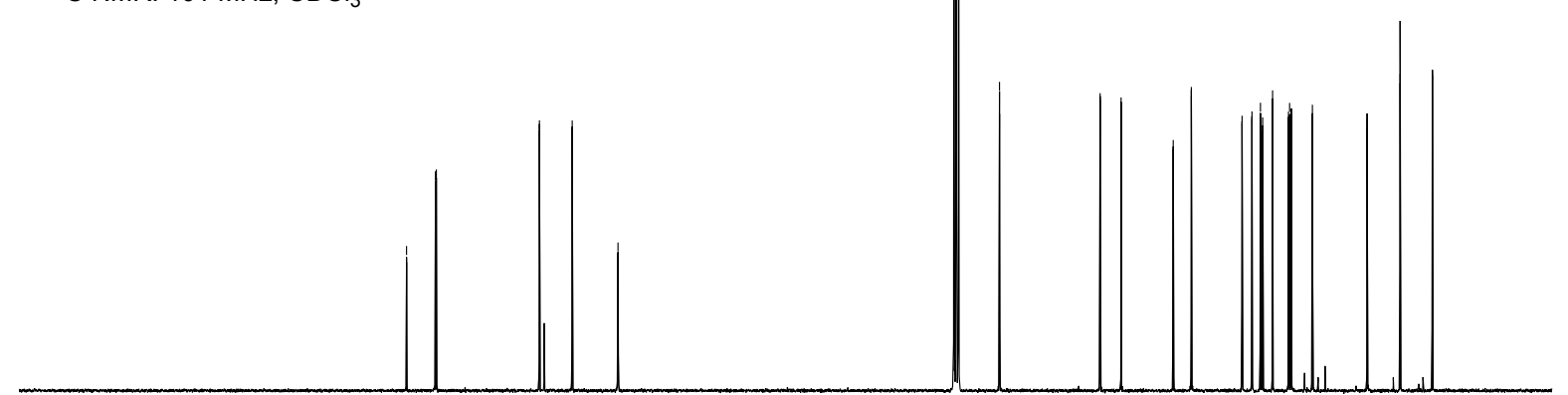

$\begin{array}{lllllllllllllllllllll}200 & 190 & 180 & 170 & 160 & 150 & 140 & 130 & 120 & 110 & \begin{array}{c}100 \\ \mathrm{ppm}\end{array} & 90 & 80 & 70 & 60 & 50 & 40 & 30 & 20 & 10 & 0\end{array}$ 
$\underline{3 \beta, 5 \alpha)-17-(4 '-P y r i d i n-2 '-y l p h e n y l) a n d r o s t-16-e n-3-o l ~(5 c) ~})^{2}$

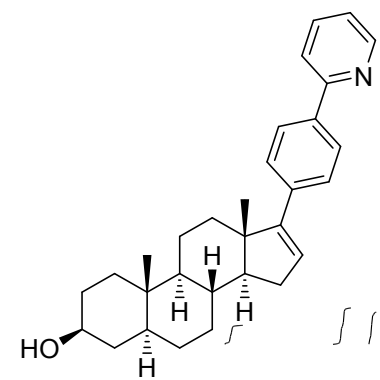

${ }^{1} \mathrm{H}$ NMR: $400 \mathrm{MHz}, \mathrm{CDCl}_{3}$

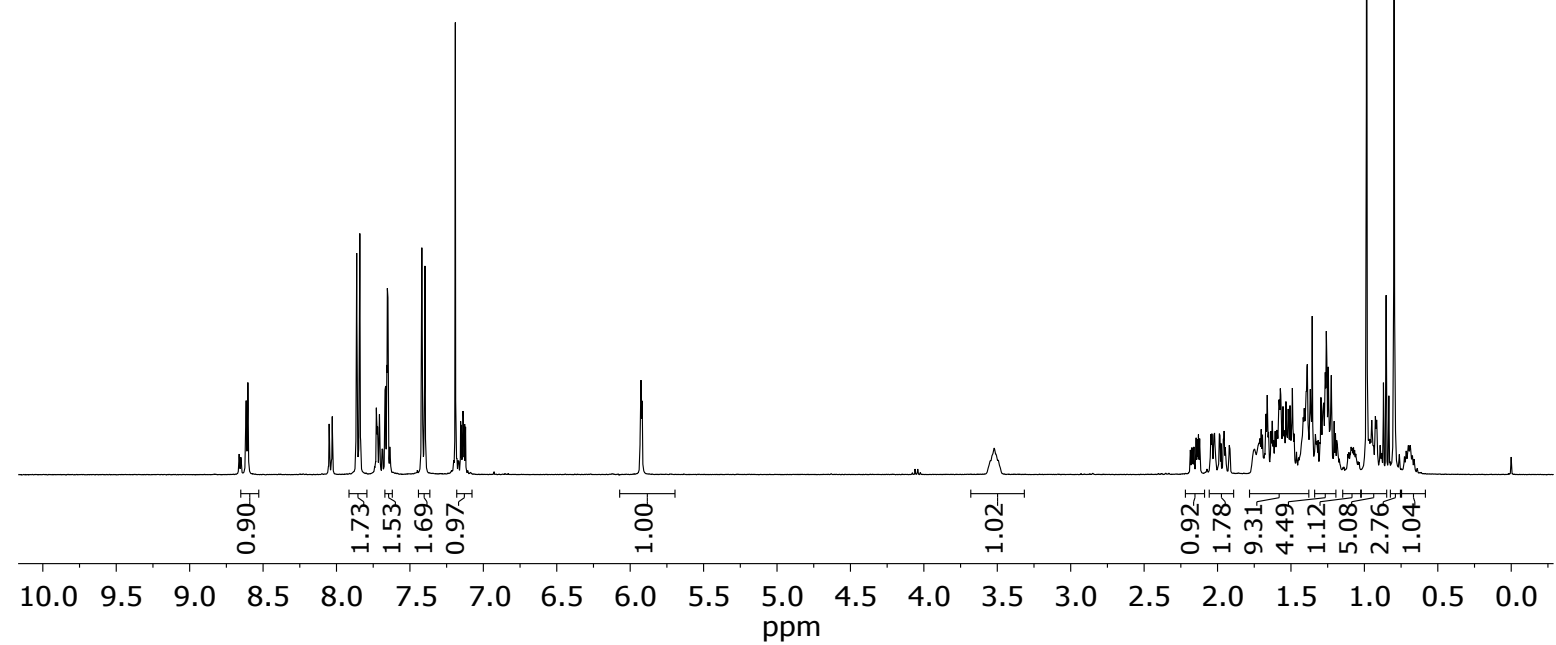

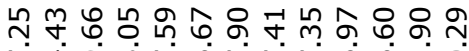

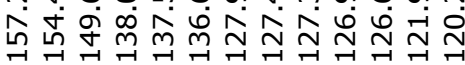

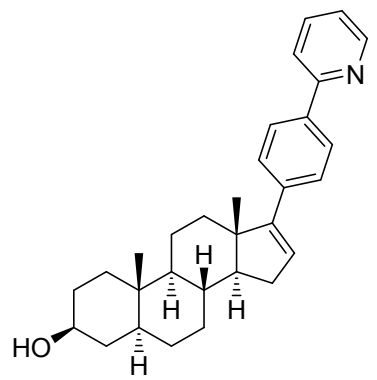

${ }^{13} \mathrm{C}$ NMR: $101 \mathrm{MHz}, \mathrm{CDCl}_{3}$

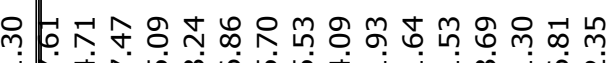

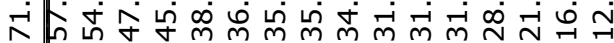

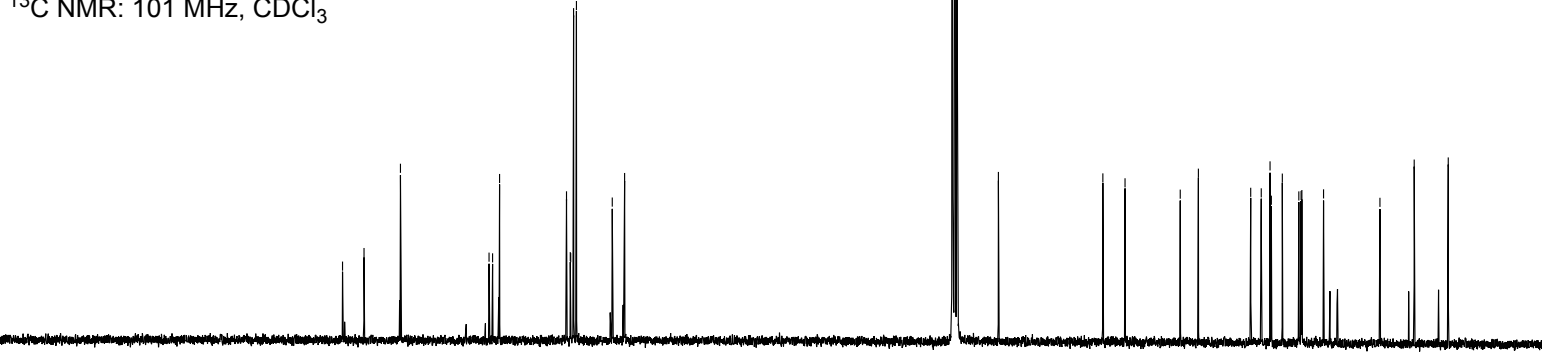


[5-Bromo-2-(2-pyridinyl- $\kappa N)$ phenyl- $\left.\kappa C^{1}\right]$ chlorido( $\eta^{6}$-para-cymene) ruthenium(II) (6)
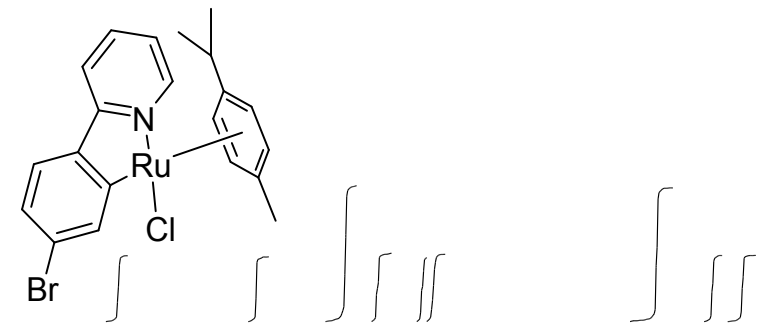

${ }^{1} \mathrm{H}$ NMR: $500 \mathrm{MHz}, \mathrm{CDCl}_{3}$

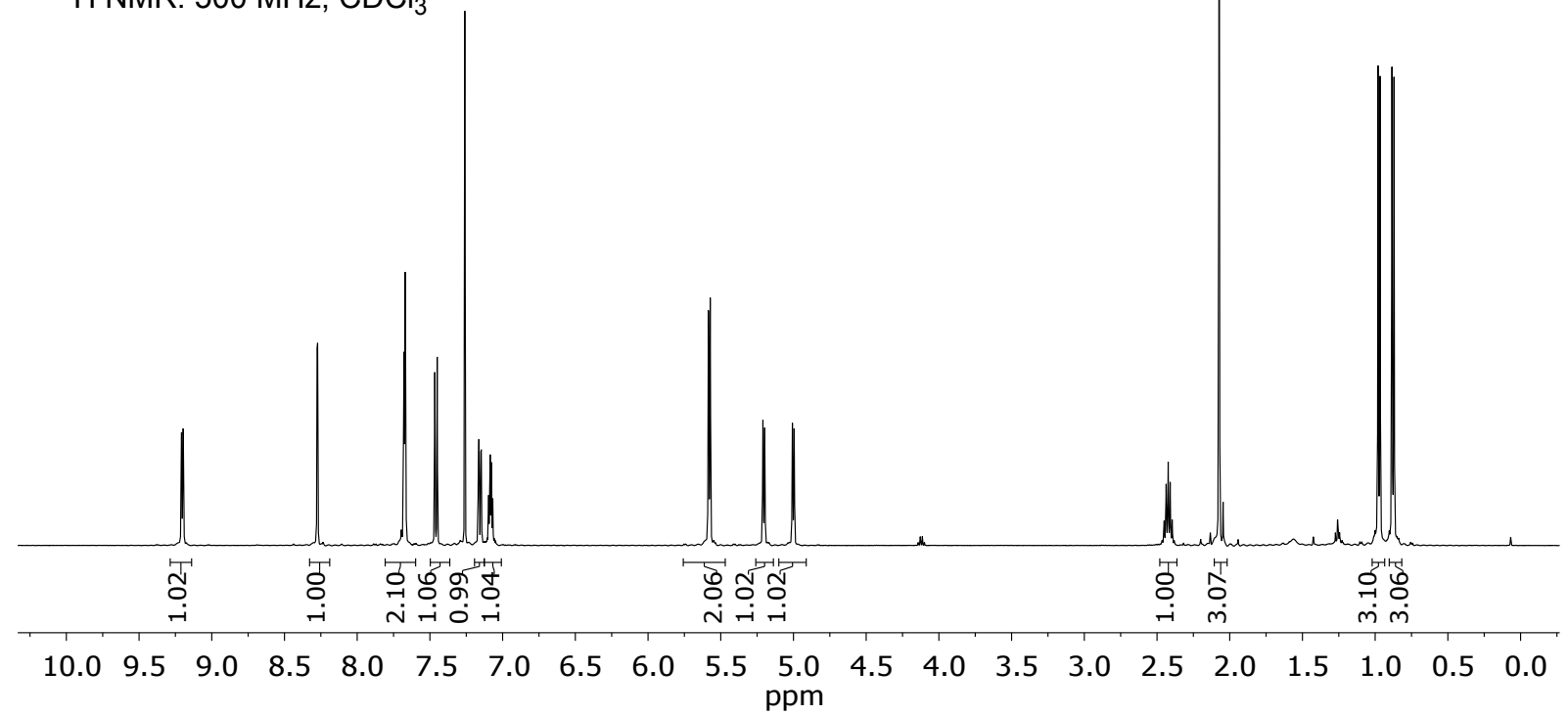

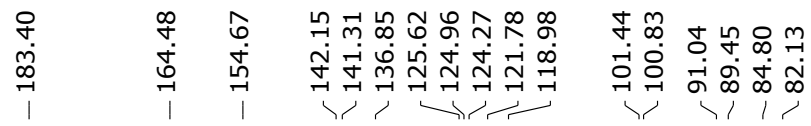

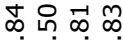

กิํํำ

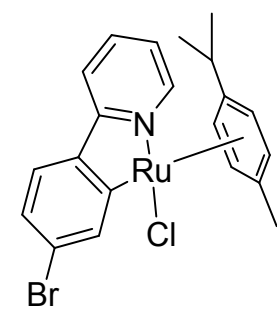

${ }^{13} \mathrm{C}$ NMR: $126 \mathrm{MHz}, \mathrm{CDCl}_{3}$

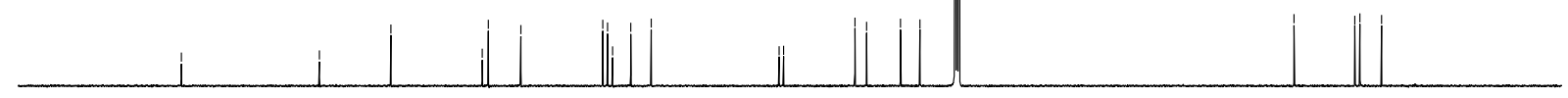

$\begin{array}{lllllllllllllllllllll}200 & 190 & 180 & 170 & 160 & 150 & 140 & 130 & 120 & 110 & \begin{array}{l}100 \\ \mathrm{ppm}\end{array} & 90 & 80 & 70 & 60 & 50 & 40 & 30 & 20 & 10 & 0\end{array}$ 
Chlorido( $\eta^{6}$-para-cymene)[17-(4'-pyridin-2 ' '-yl- $\kappa N$-phenyl-3 $\left.{ }^{\prime} \kappa C\right)-(5 \alpha, 3 \beta)$-androst-16-enolido]ruthenium(II) (7)

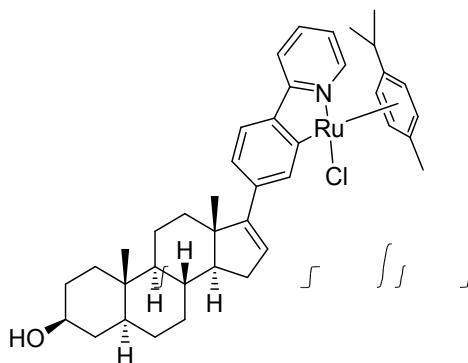

${ }^{1} \mathrm{H}$ NMR: $600 \mathrm{MHz}, \mathrm{CDCl}_{3}$

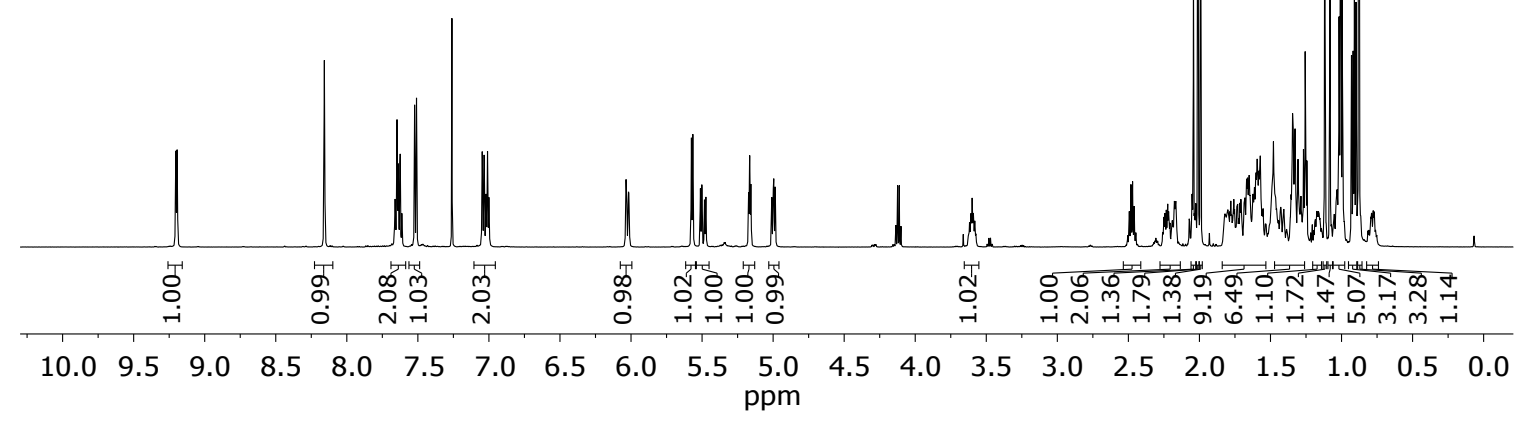

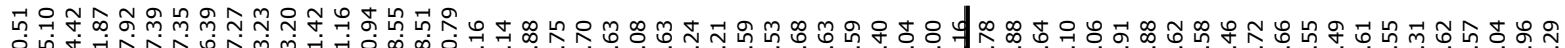

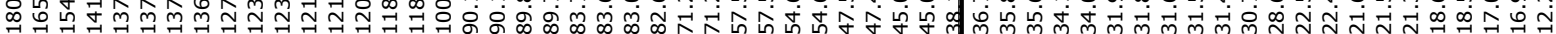

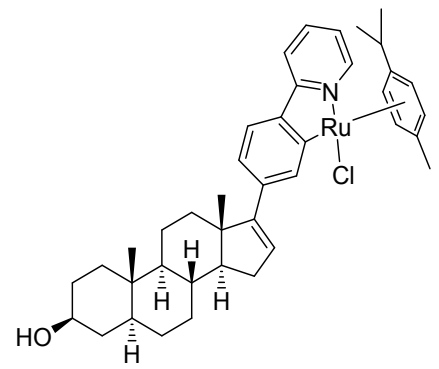

${ }^{13} \mathrm{C}$ NMR: $151 \mathrm{MHz}, \mathrm{CDCl}_{3}$

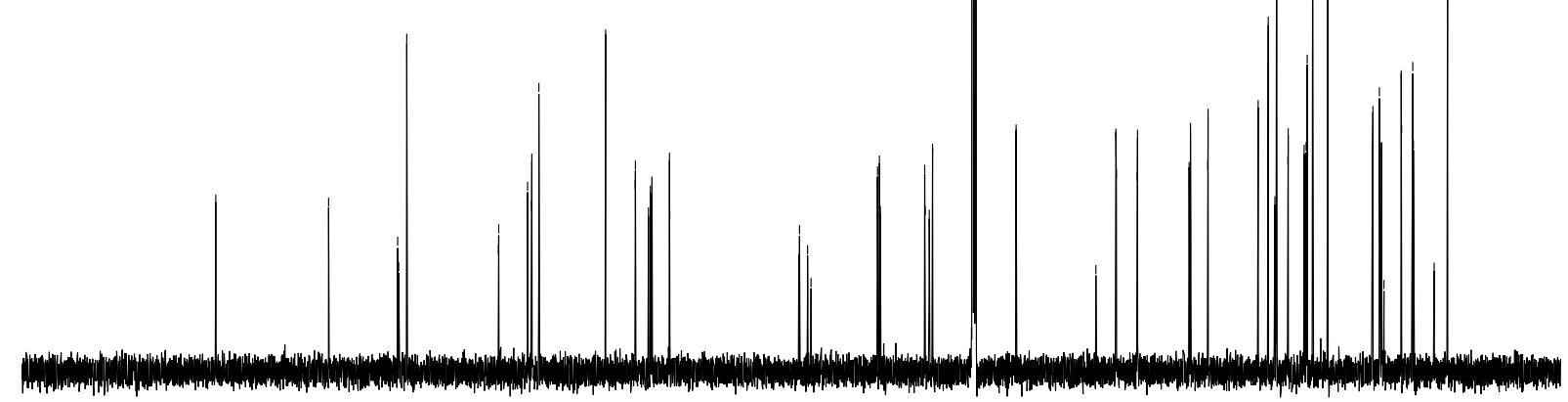

$\begin{array}{lllllllllllllllllllll}200 & 190 & 180 & 170 & 160 & 150 & 140 & 130 & 120 & 110 & \begin{array}{c}100 \\ \mathrm{ppm}\end{array} & 90 & 80 & 70 & 60 & 50 & 40 & 30 & 20 & 10 & 0\end{array}$ 


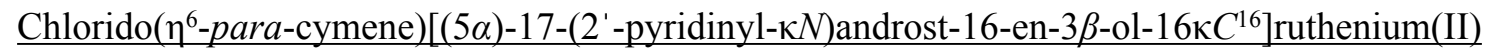

\section{$\underline{((R-R u)-8)}$}

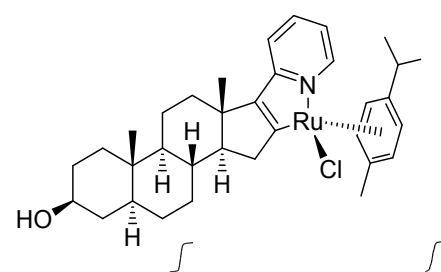

${ }^{1} \mathrm{H}$ NMR: $500 \mathrm{MHz}, \mathrm{CDCl}_{3}$
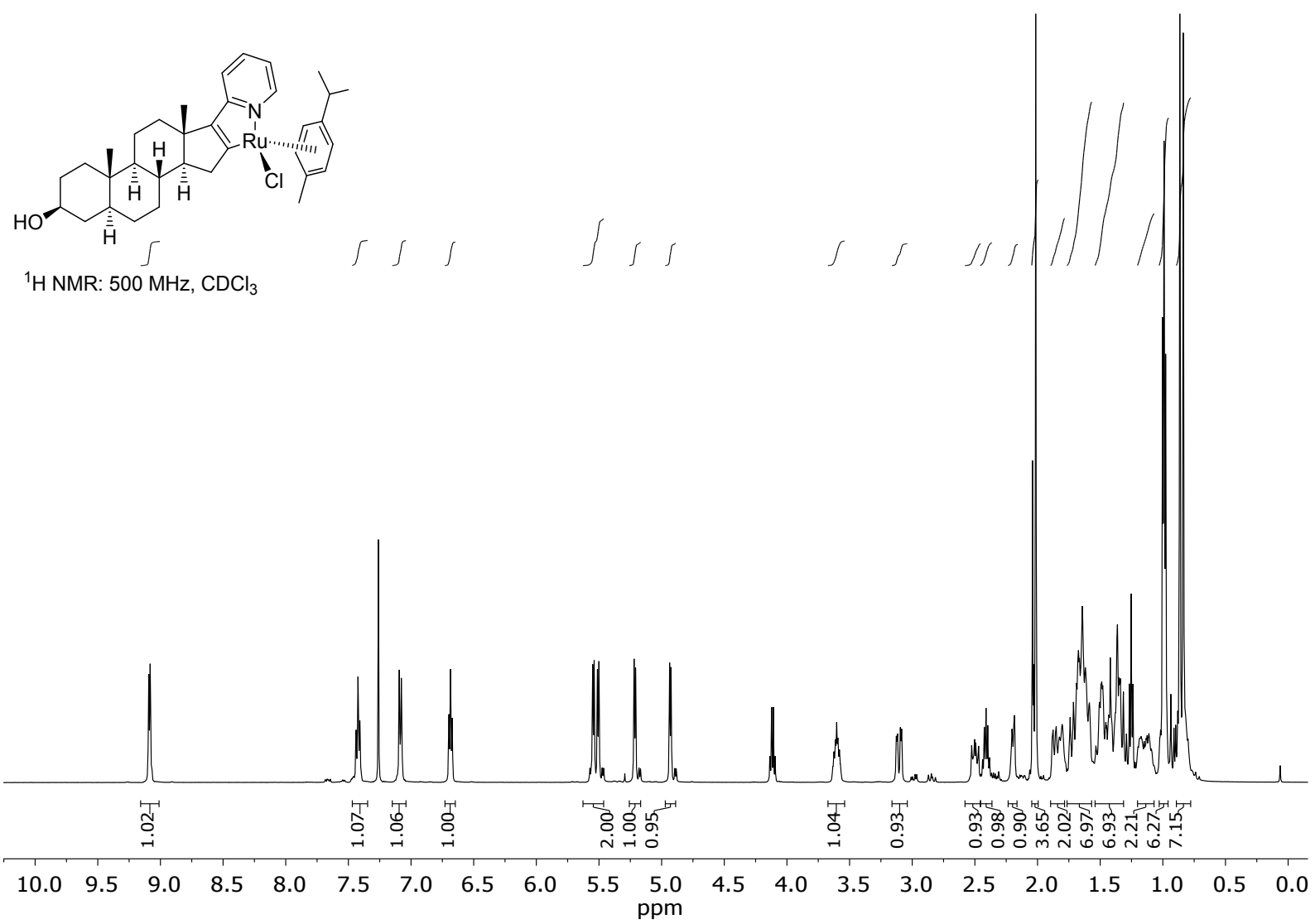

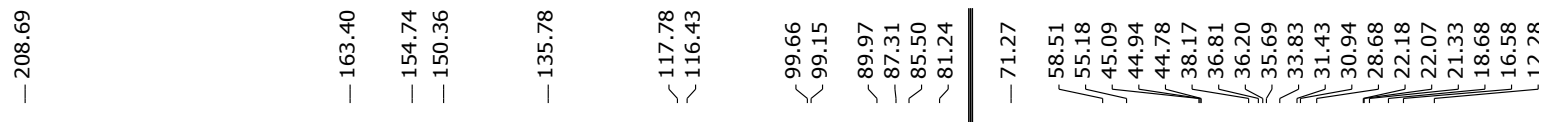

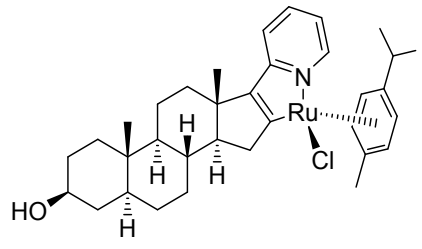

${ }^{13} \mathrm{C}$ NMR: $126 \mathrm{MHz}, \mathrm{CDCl}_{3}$

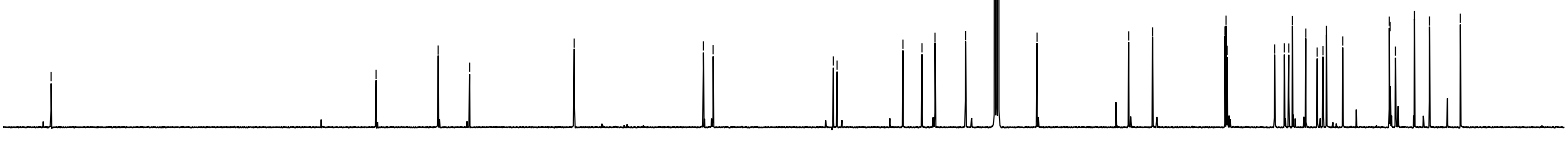

$\begin{array}{llllllllllllllllllllll}210 & 200 & 190 & 180 & 170 & 160 & 150 & 140 & 130 & 120 & 110 & 100 & 90 & 80 & 70 & 60 & 50 & 40 & 30 & 20 & 10 & 0\end{array}$ 


\section{$\underline{((S-R u)-8)}$}

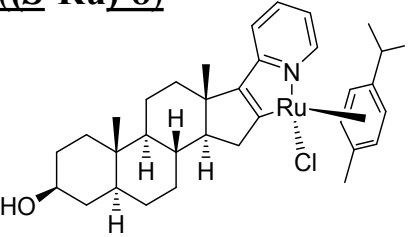

${ }^{1} \mathrm{H}$ NMR: $500 \mathrm{MHz}, \mathrm{CDCl}_{3}$
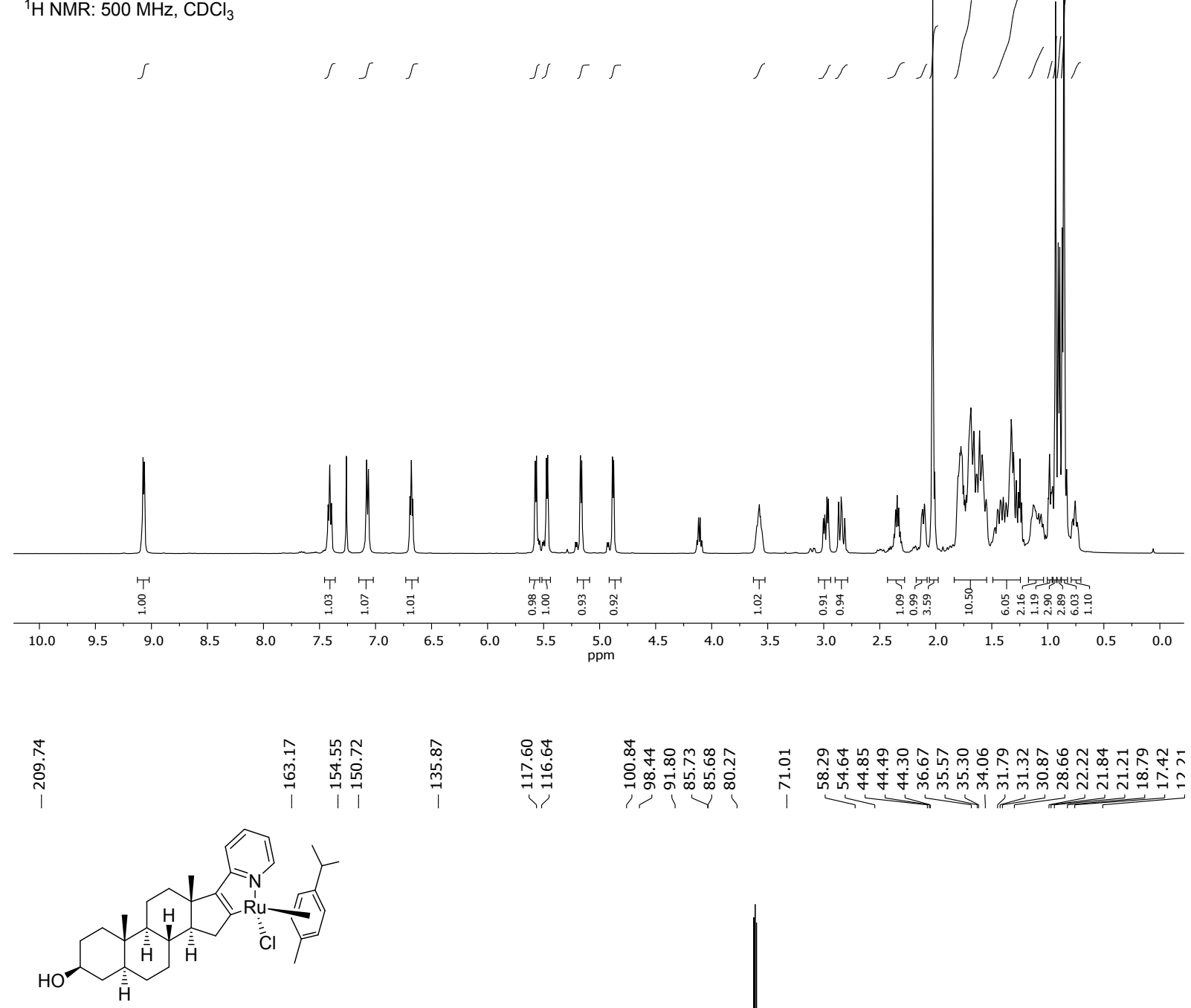

${ }^{13} \mathrm{C}$ NMR: $126 \mathrm{MHz}, \mathrm{CDCl}_{3}$

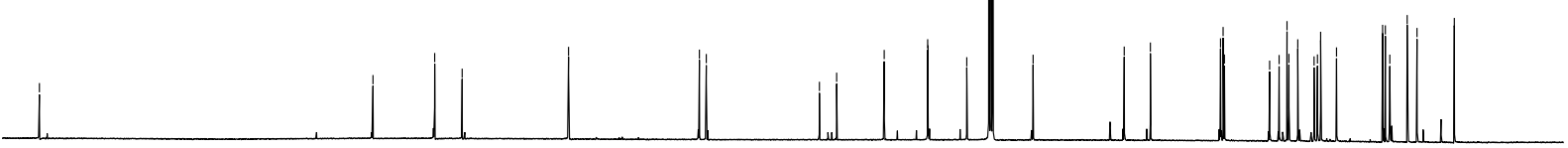

$\begin{array}{llllllllllllllllllllllllll}210 & 200 & 190 & 180 & 170 & 160 & 150 & 140 & 130 & 120 & 110 & 100 & 90 & 80 & 70 & 60 & 50 & 40 & 30 & 20 & 10 & 0\end{array}$ 


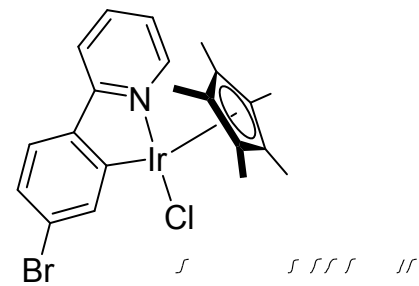

${ }^{1} \mathrm{H} \mathrm{NMR}\left(500 \mathrm{MHz}, \mathrm{CDCl}_{3}\right)$
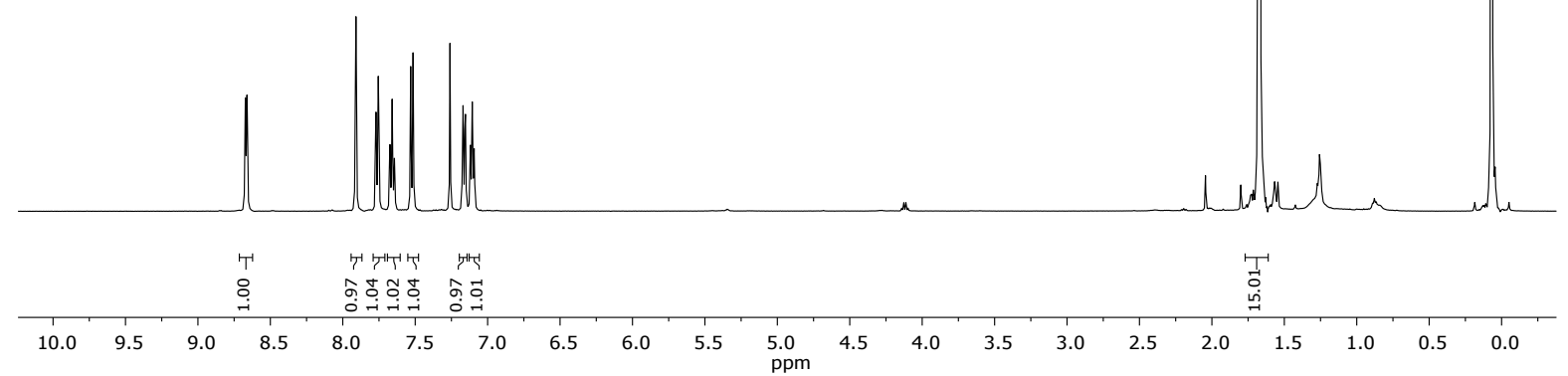

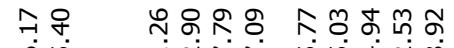

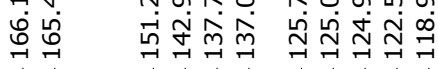
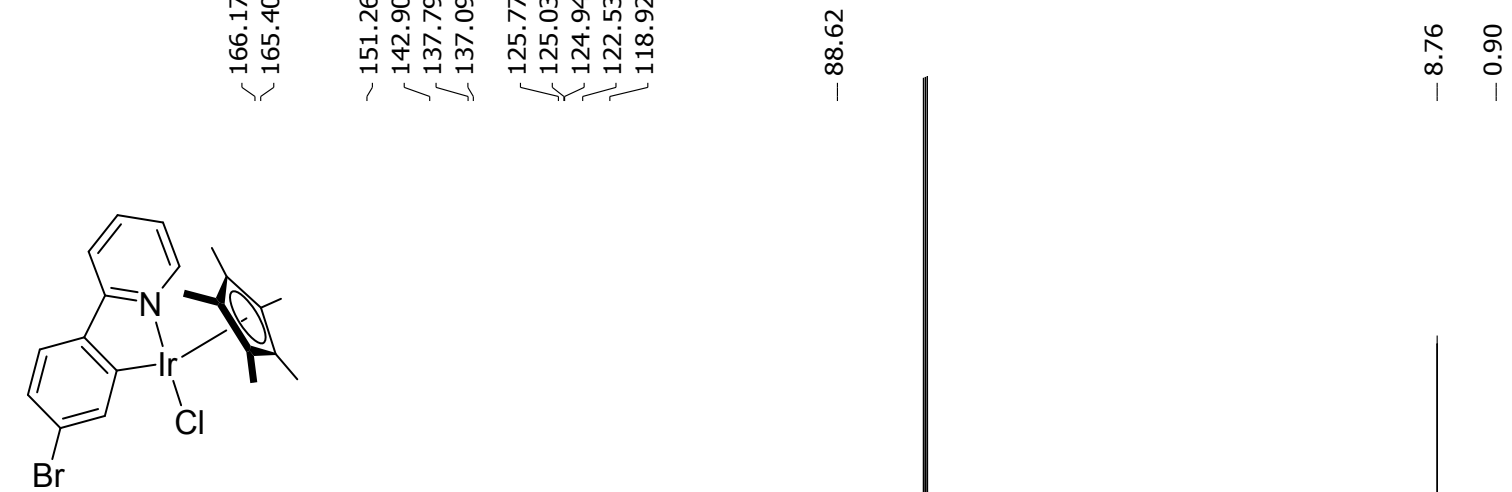

${ }^{13} \mathrm{C}$ NMR $\left(126 \mathrm{MHz}, \mathrm{CDCl}_{3}\right)$

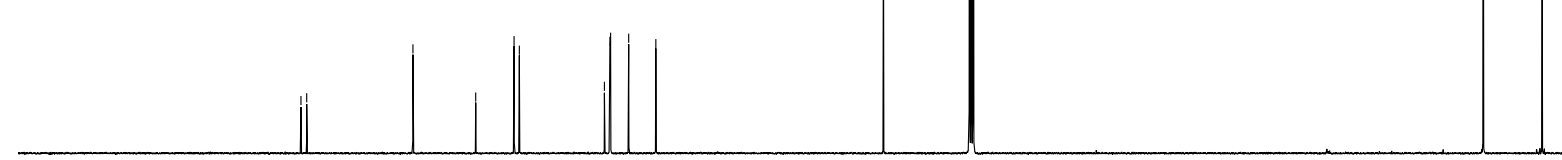

$\begin{array}{lllllllllllllllllllllll}200 & 190 & 180 & 170 & 160 & 150 & 140 & 130 & 120 & 110 & 100 & 90 & 80 & 70 & 60 & 50 & 40 & 30 & 20 & 10 & 0\end{array}$ 
Chlorido( $\eta^{5}$-pentamethylcyclopentadienyl)[17-(4'-pyridin-2 ' '-yl- $\kappa \mathrm{N}$-phenyl-3' $\left.\kappa \mathrm{C}\right)-(5 \alpha, 3 \beta)$ androst-16enolido]iridium(III) (10)

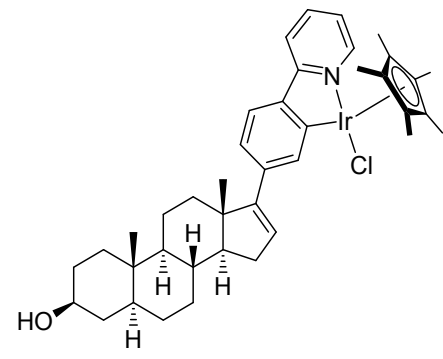

${ }^{1} \mathrm{H}$ NMR: $500 \mathrm{MHz}, \mathrm{CDCl}_{3}$
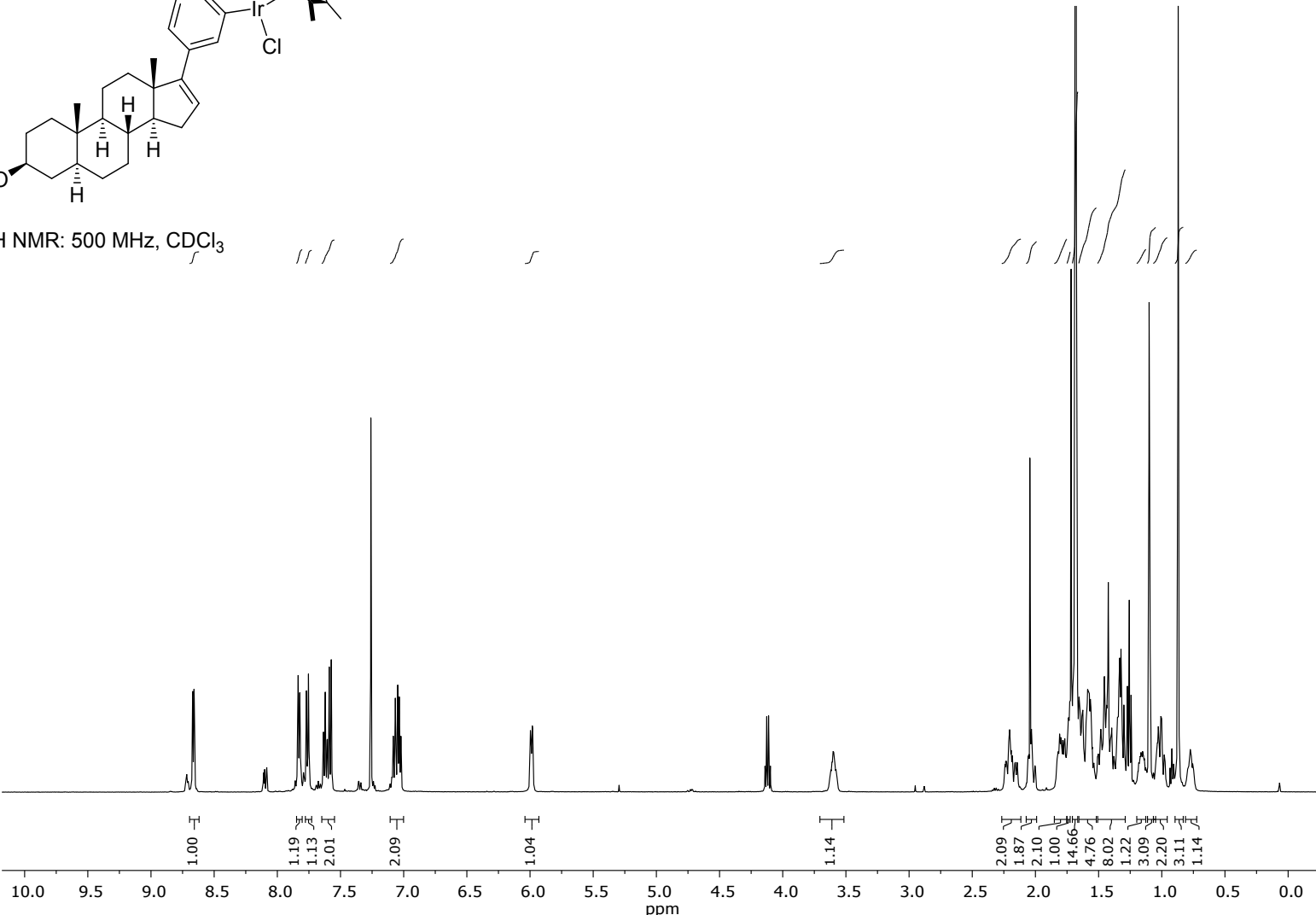

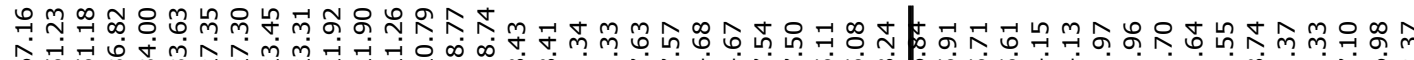

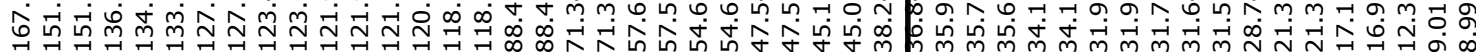

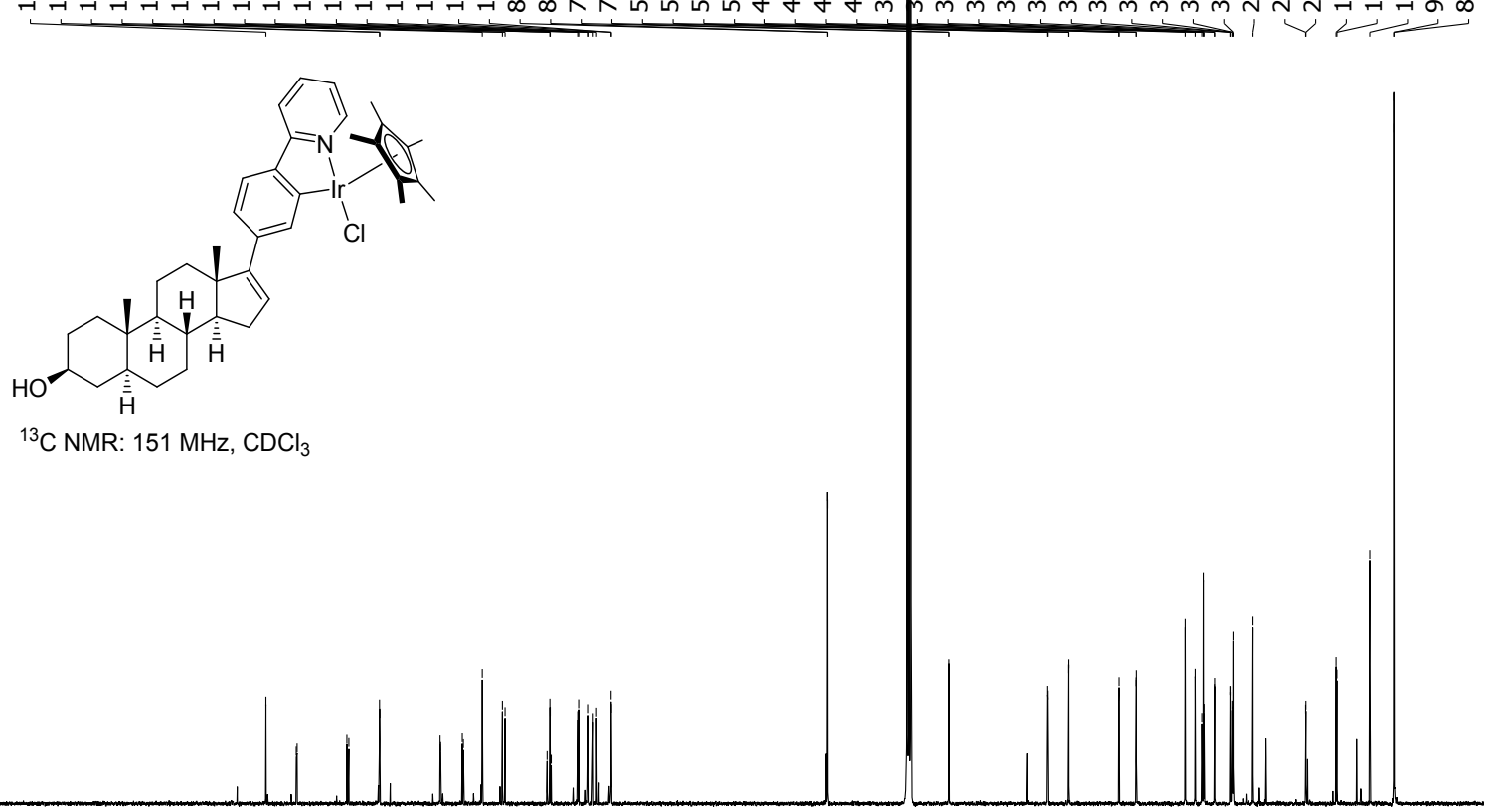

$\begin{array}{lllllllllllllllllllll}200 & 190 & 180 & 170 & 160 & 150 & 140 & 130 & 120 & 110 & \begin{array}{c}100 \\ \mathrm{ppm}\end{array} & 90 & 80 & 70 & 60 & 50 & 40 & 30 & 20 & 10 & 0\end{array}$ 


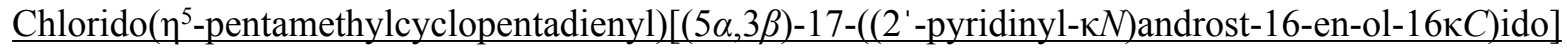
iridium(III) (11)

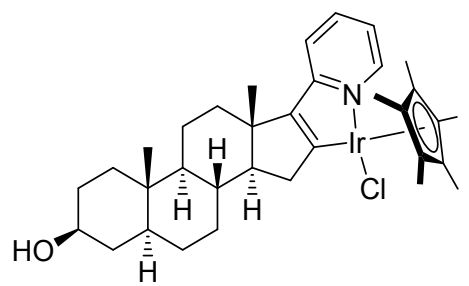

${ }^{1} \mathrm{H}$ NMR: $400 \mathrm{MHz}, \mathrm{CDCl}_{3}$

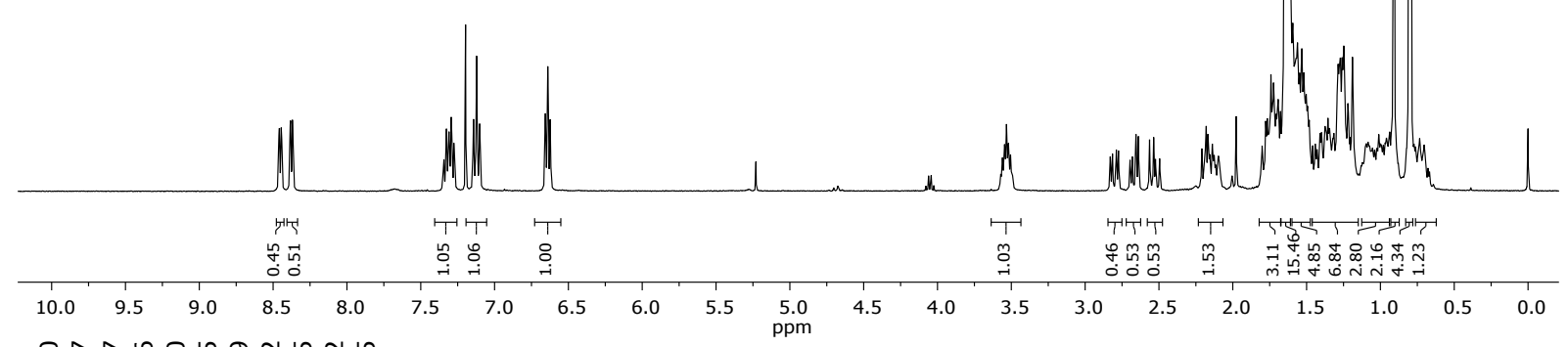

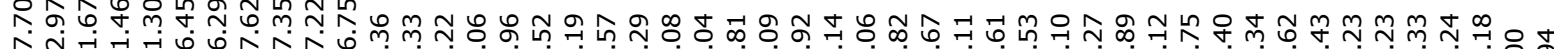

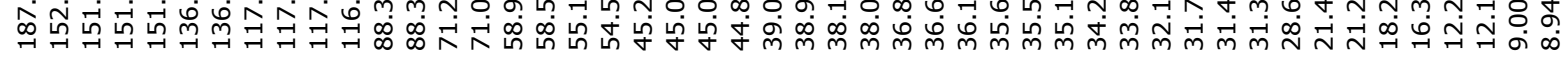

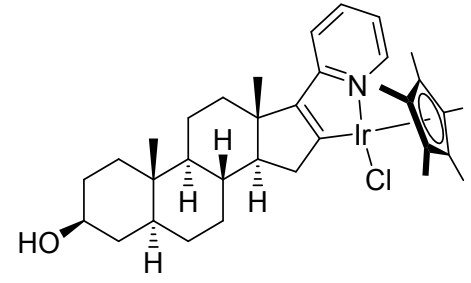

${ }^{13} \mathrm{C}$ NMR: $101 \mathrm{MHz}, \mathrm{CDCl}_{3}$

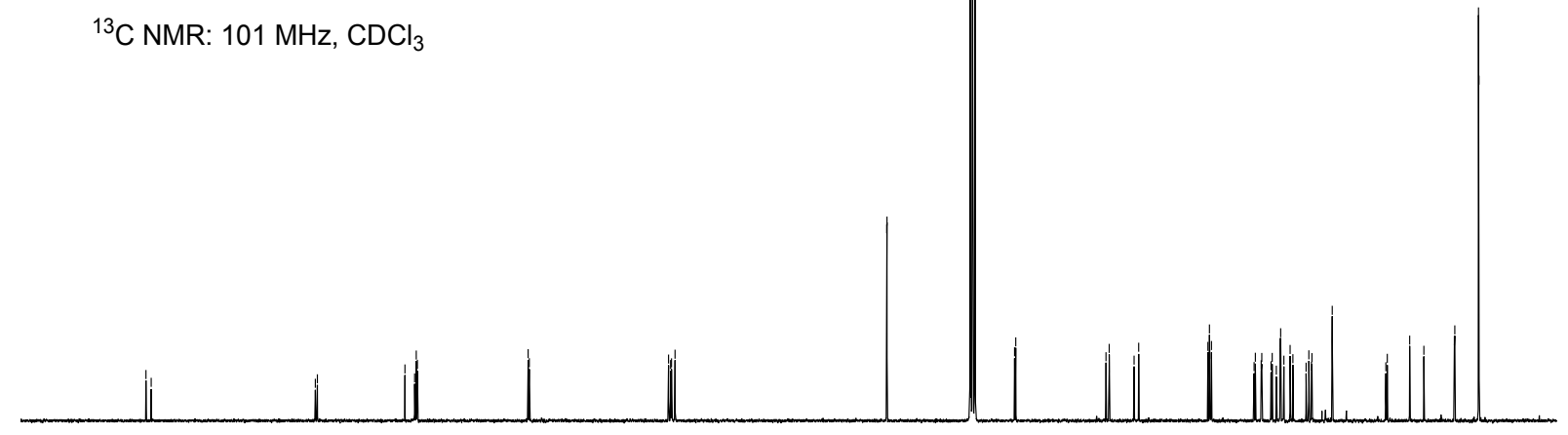

$\begin{array}{lllllllllllllllllllll}200 & 190 & 180 & 170 & 160 & 150 & 140 & 130 & 120 & 110 & \begin{array}{c}100 \\ \mathrm{ppm}\end{array} & 90 & 80 & 70 & 60 & 50 & 40 & 30 & 20 & 10 & 0\end{array}$ 
Dichlorido( $\eta^{6}$-para-cymene) $\left[(5 \alpha, 3 \beta)-17-\left(\left(3^{\prime}\right.\right.\right.$-pyridinyl- $\left.\kappa \mathrm{N}\right)$-androst-16-en-3-ol)ido]ruthenium(II) (12)

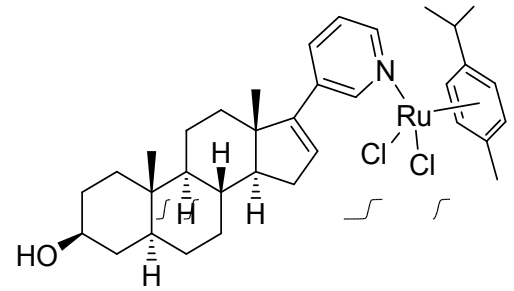

${ }^{1} \mathrm{H}$ NMR: $500 \mathrm{MHz}, \mathrm{CDCl}_{3}$

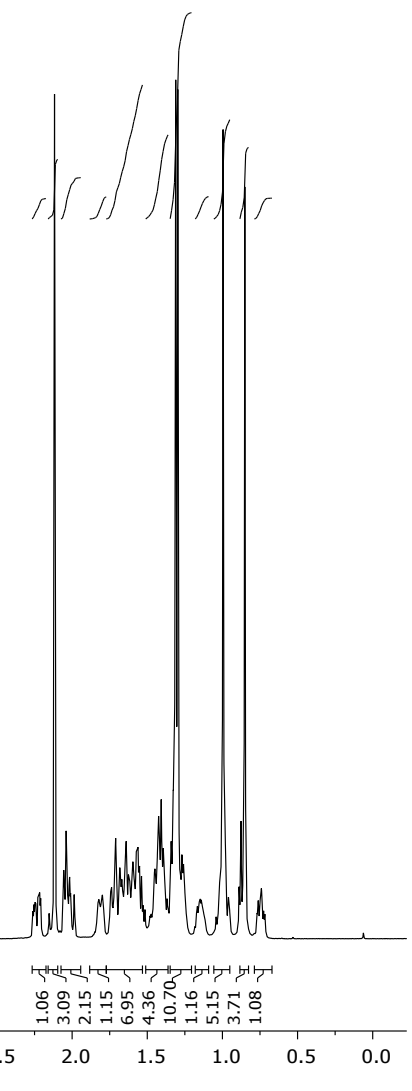

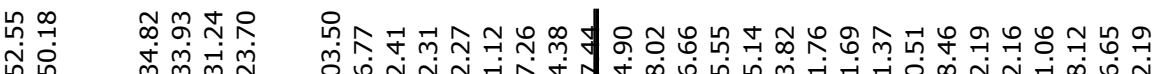

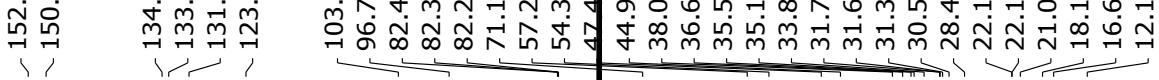

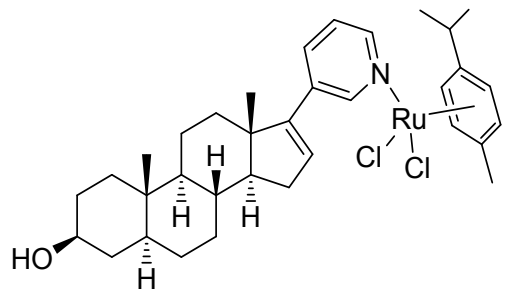

${ }^{13} \mathrm{C} \mathrm{NMR:} 126 \mathrm{MHz}, \mathrm{CDCl}_{3}$

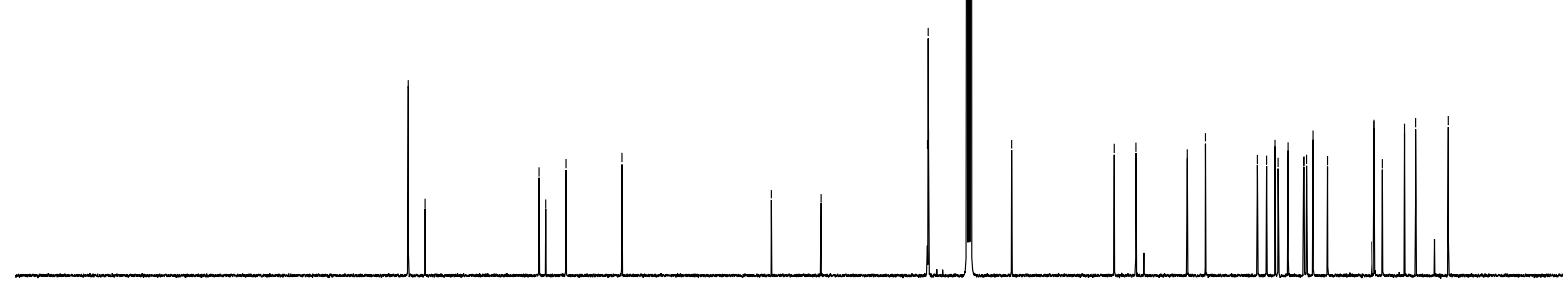

$\begin{array}{lllllllllllllllllllll}200 & 190 & 180 & 170 & 160 & 150 & 140 & 130 & 120 & 110 & 100 & 90 & 80 & 70 & 60 & 50 & 40 & 30 & 20 & 10 & 0\end{array}$ 
Dichlorido( $\eta^{5}$-pentamethylcyclopentadienyl $)\left[(5 \alpha, 3 \beta)-17-\left(\left(3^{\prime}\right.\right.\right.$-pyridinyl- $\left.\kappa \mathrm{N}\right)$ androst-16-en-3-ol $)$ ido $]-$ iridium(III) (13)

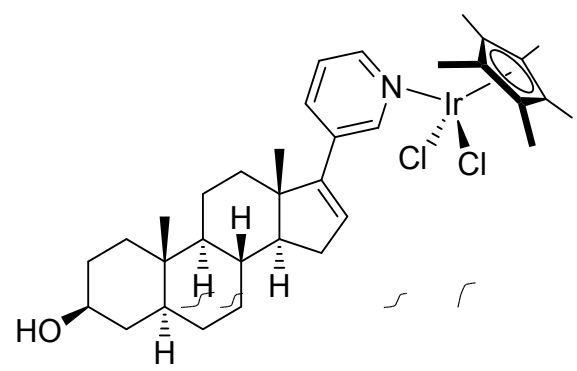

${ }^{1} \mathrm{H}$ NMR $\left(500 \mathrm{MHz}, \mathrm{CDCl}_{3}\right)$

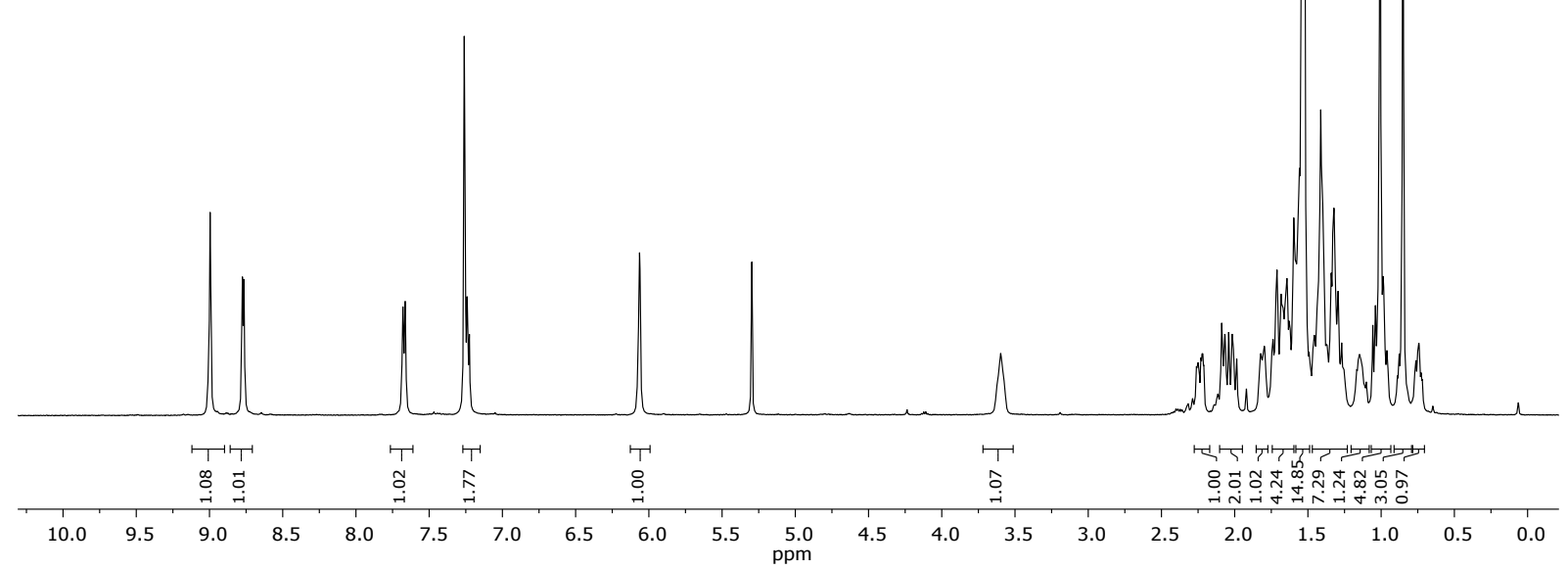

ำ

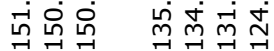

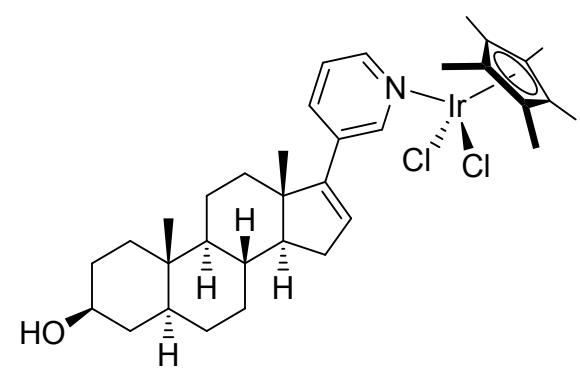

${ }^{13} \mathrm{C} \mathrm{NMR}\left(126 \mathrm{MHz}, \mathrm{CDCl}_{3}\right)$
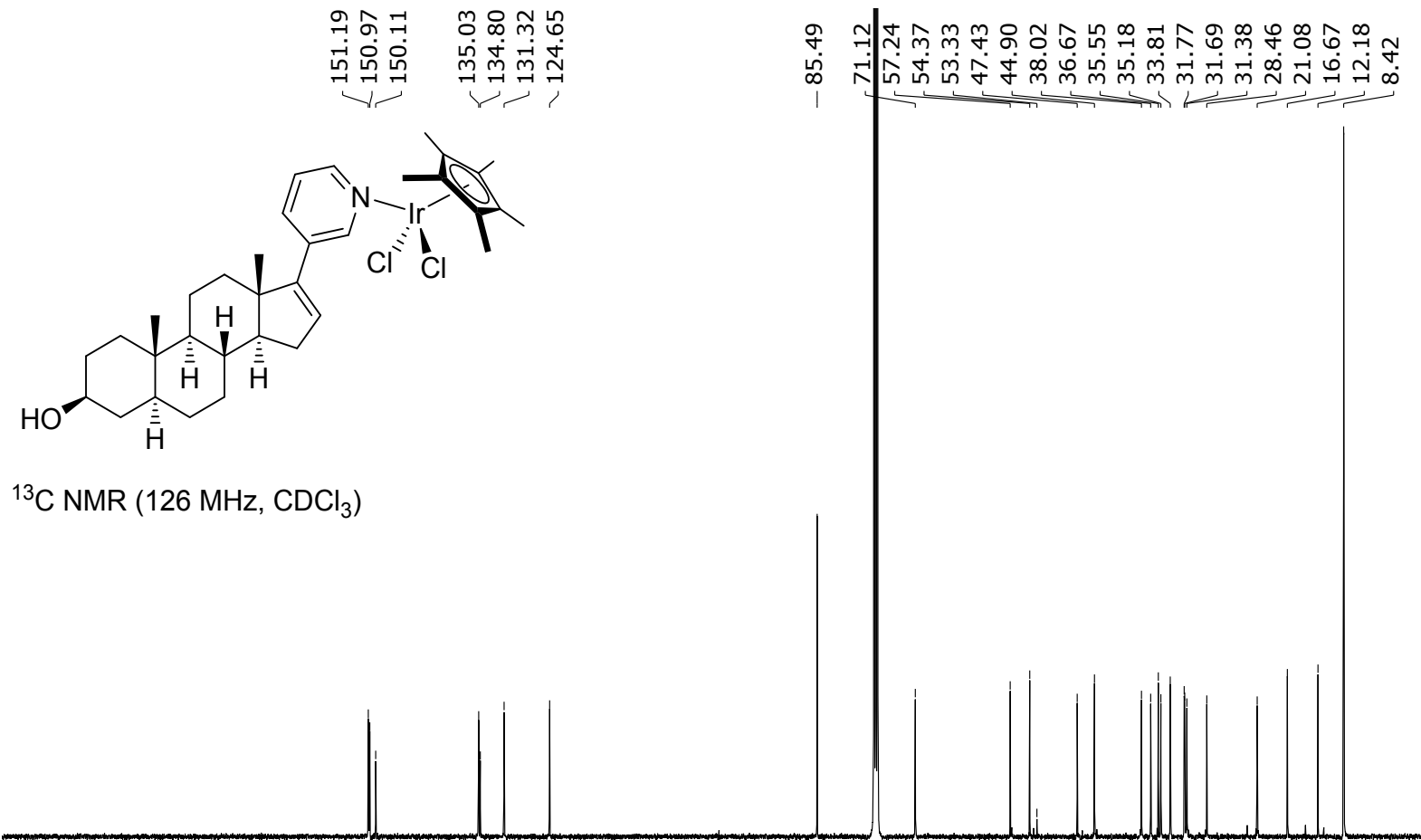

$\begin{array}{lllllllllllllllllllll}200 & 190 & 180 & 170 & 160 & 150 & 140 & 130 & 120 & 110 & \begin{array}{c}100 \\ \mathrm{ppm}\end{array} & 90 & 80 & 70 & 60 & 50 & 40 & 30 & 20 & 10 & 0\end{array}$ 


\subsection{Additional 2D NMR spectra for selected compounds}

COSY spectra of Ru-complex 7:

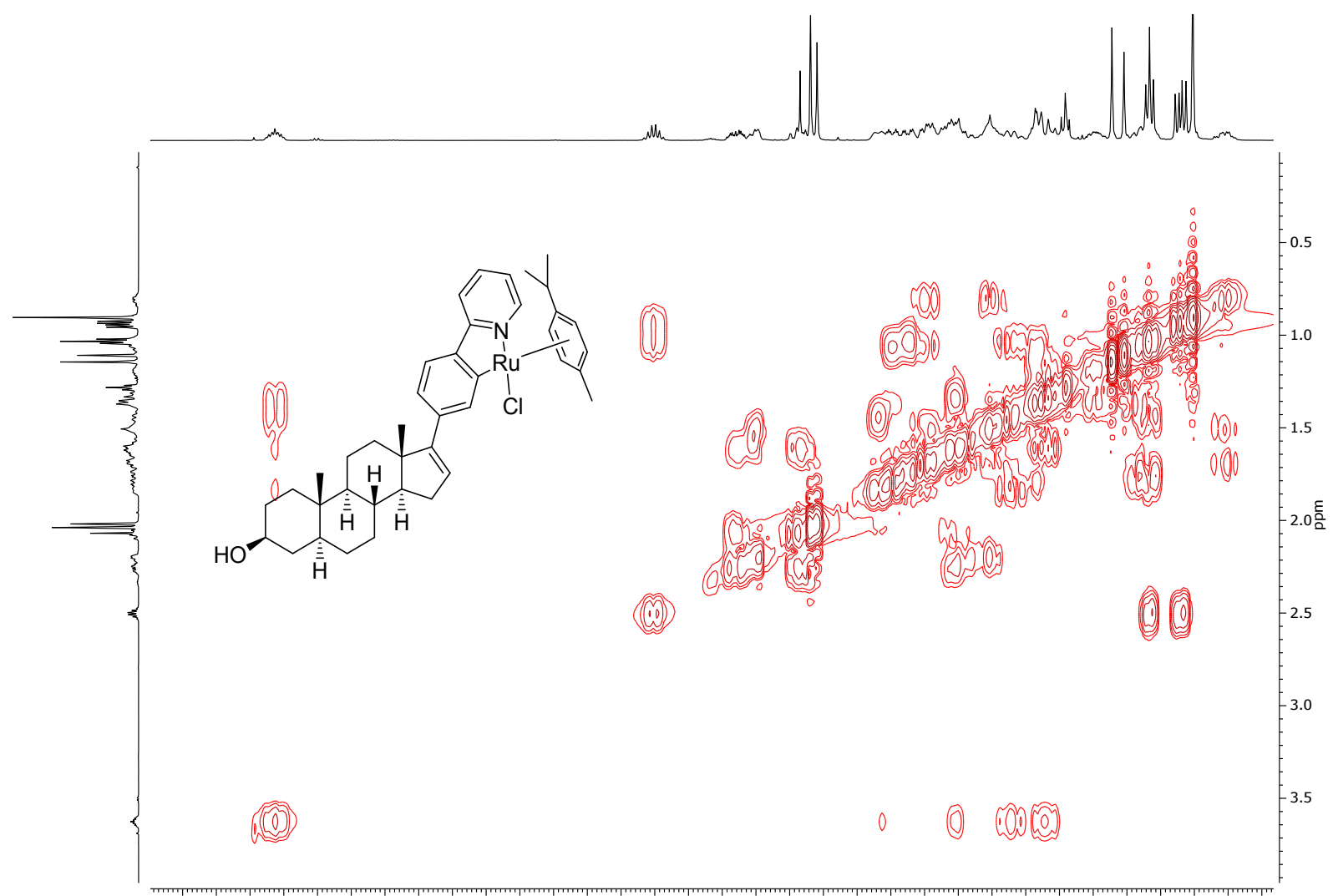

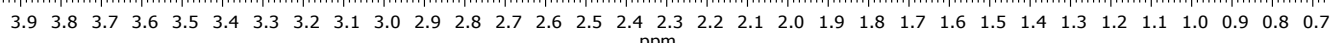

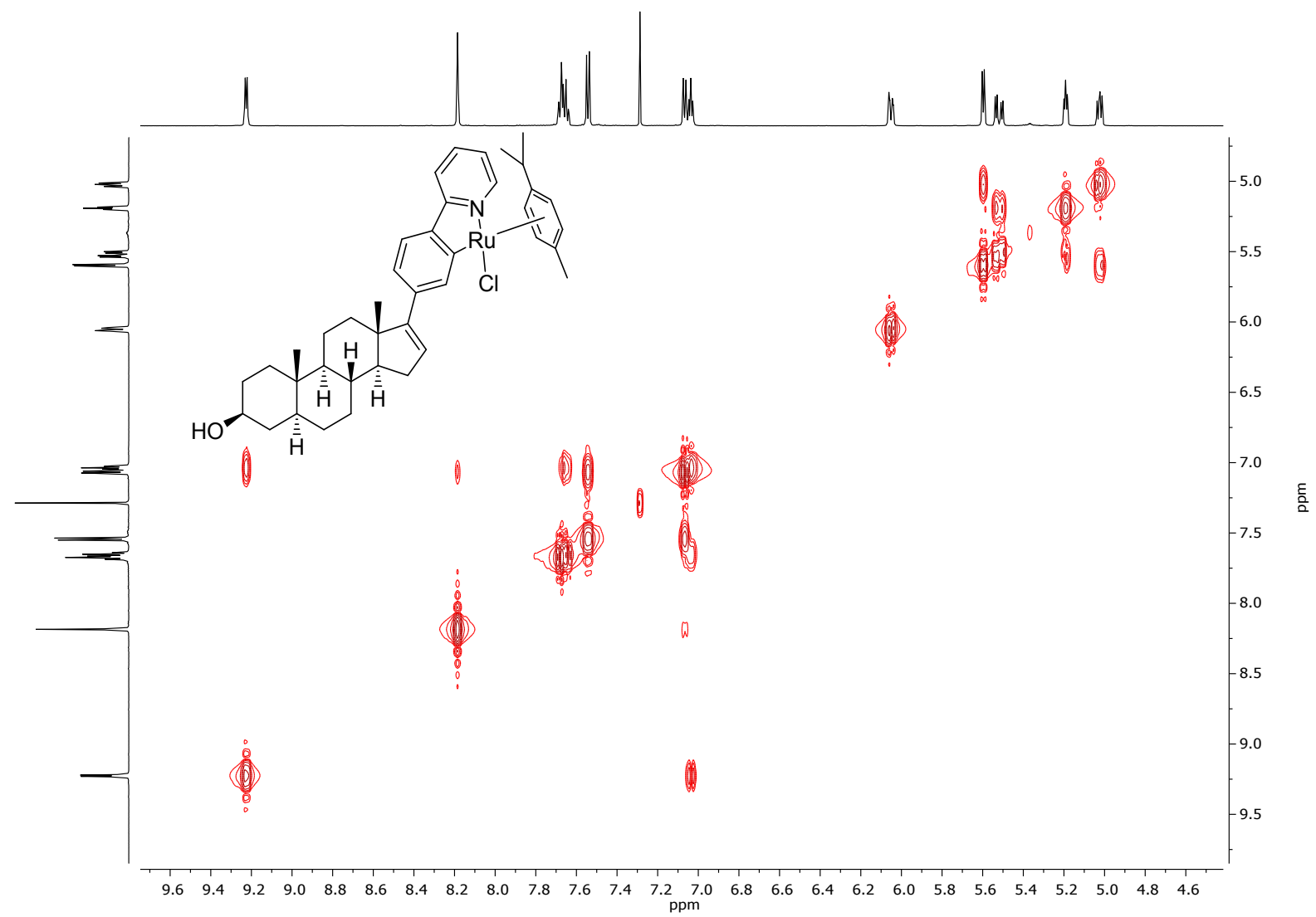


HSQC spectra of Ru-complex 7:
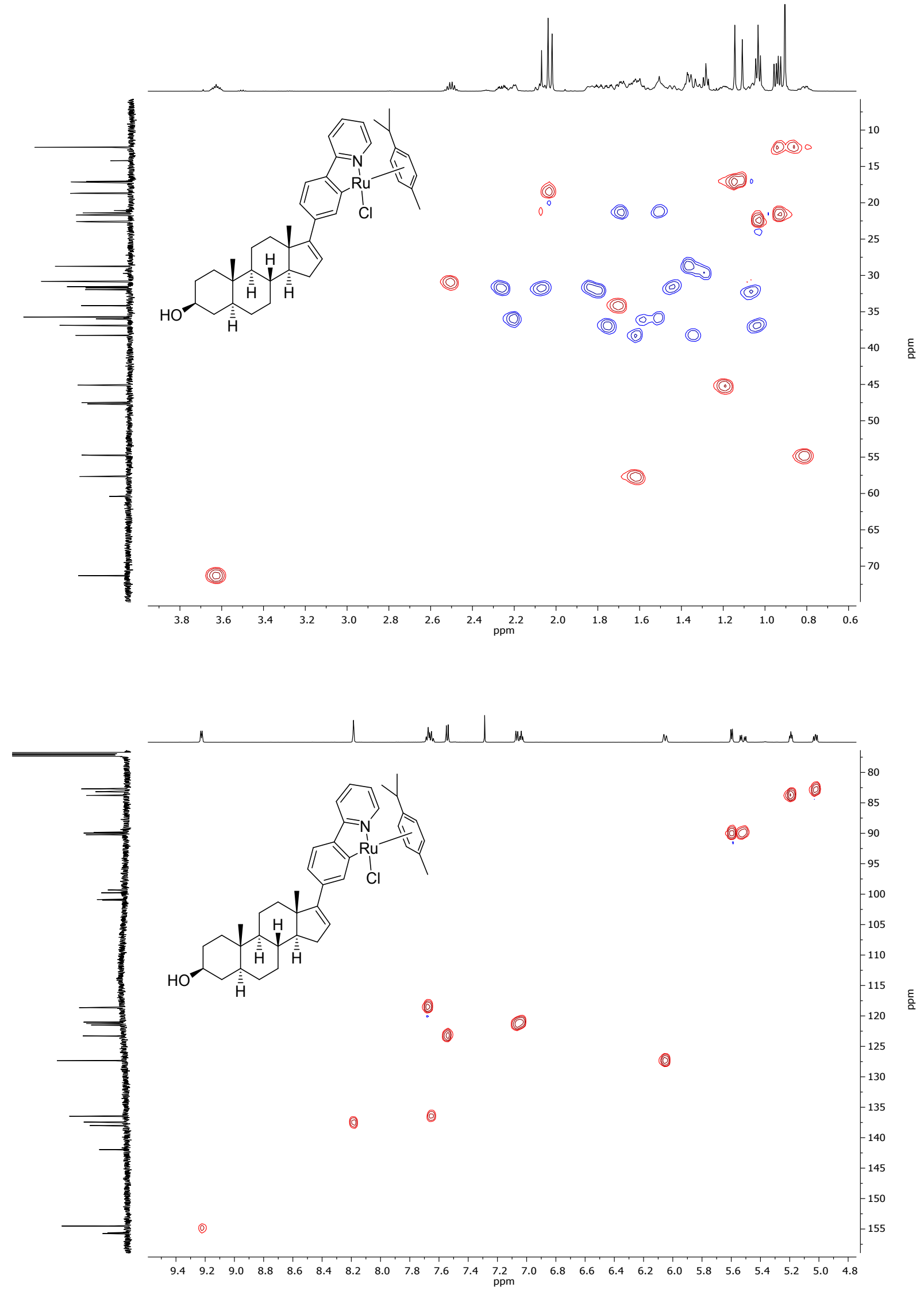
HMBC spectrum of Ru-complex 7:

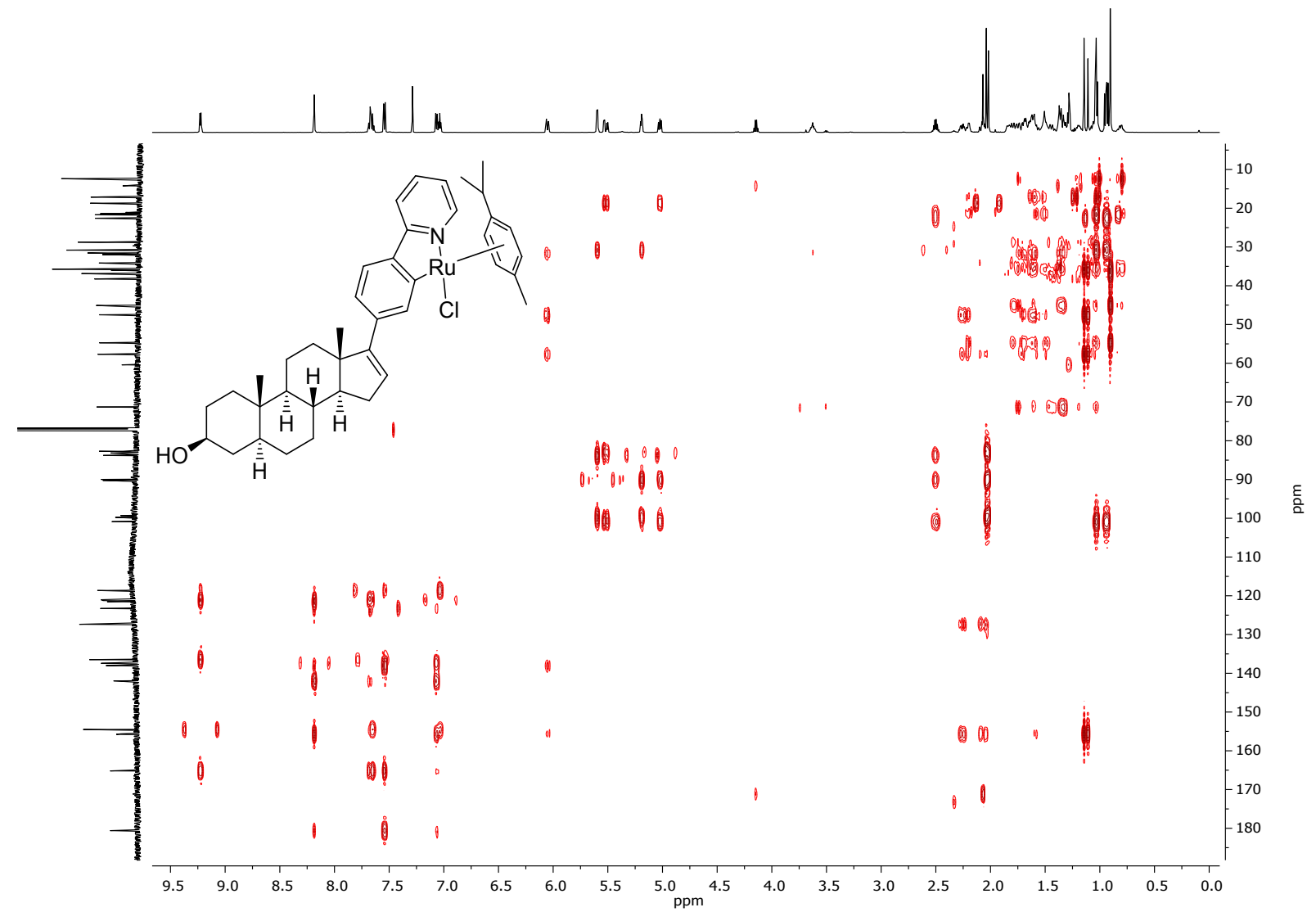


COSY spectra of Ru-complex ( $R-\mathbf{R u})-8$ :
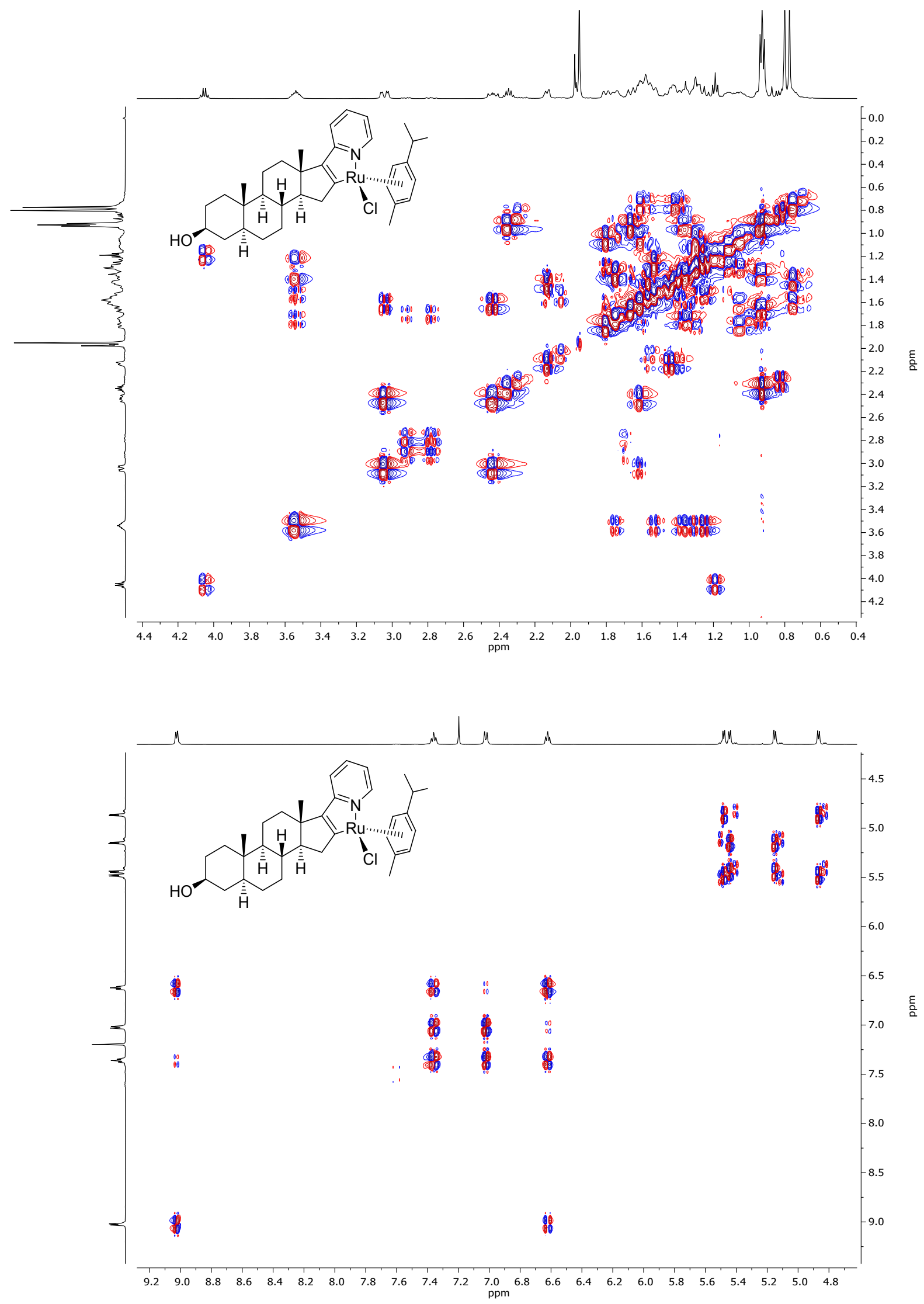
NOESY spectra of Ru-complex ( $R-\mathbf{R u})-8$ :
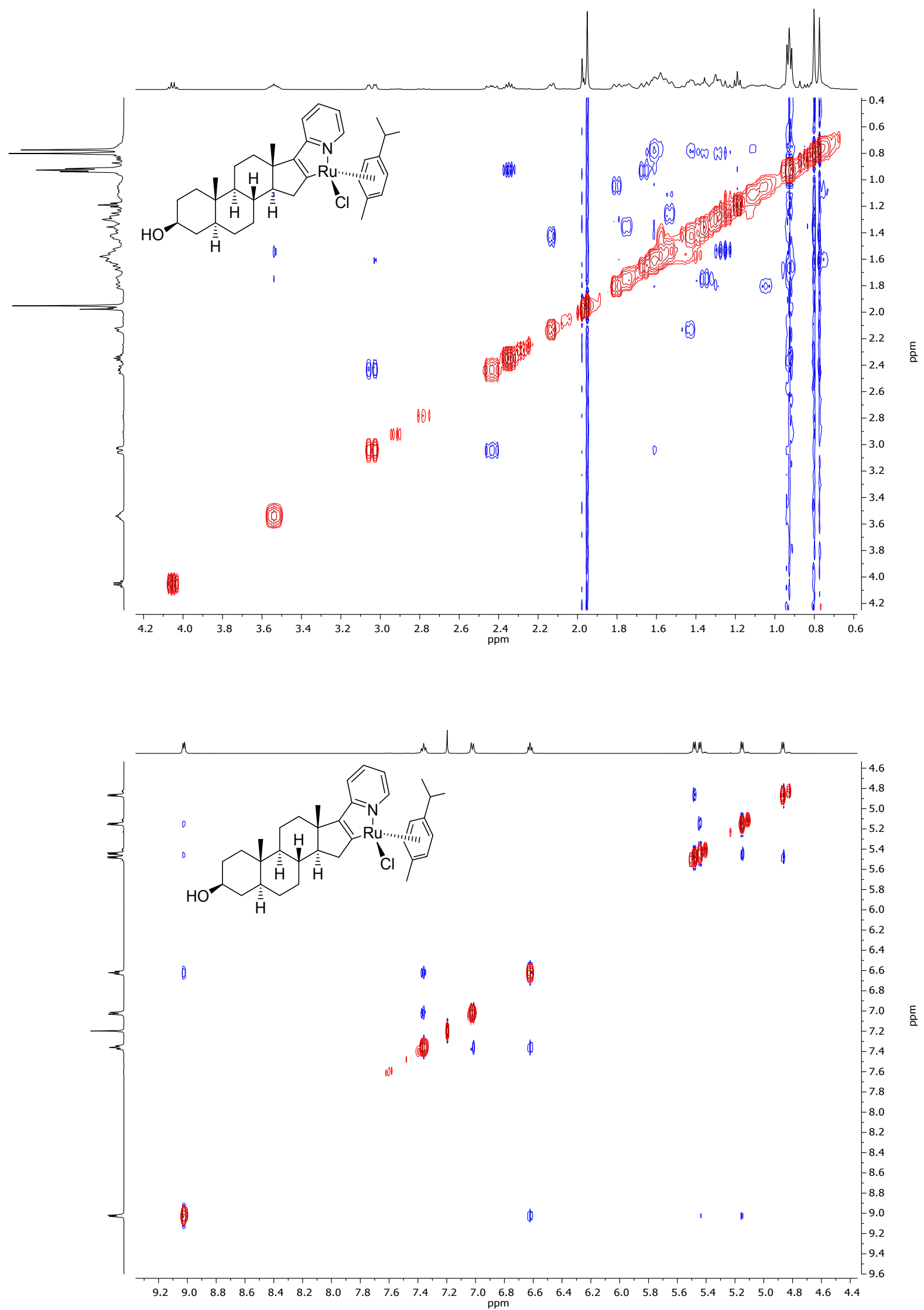
HSQC spectra of Ru-complex ( $R-\mathbf{R u})-8$ :
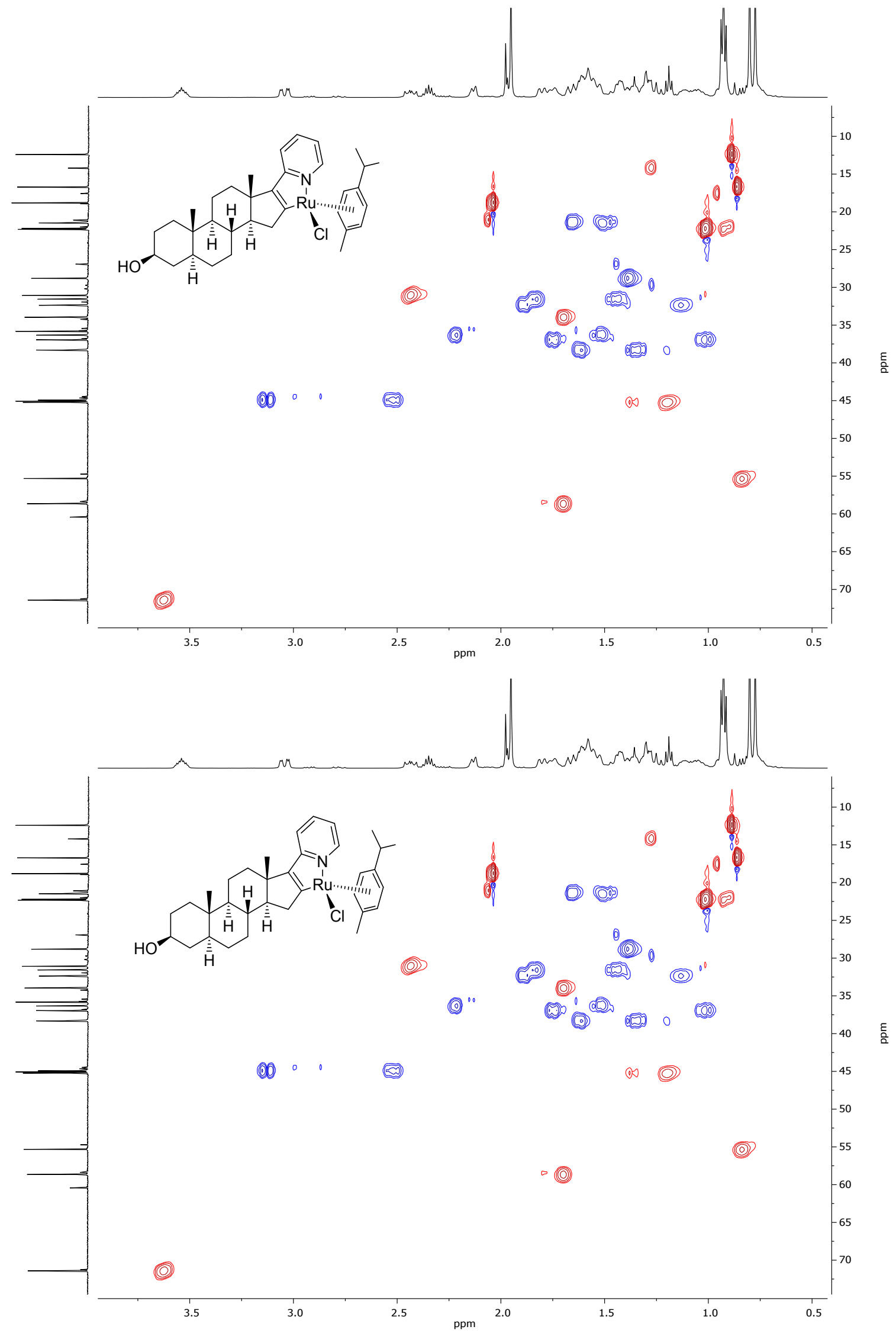
HMBC spectrum of Ru-complex (R-Ru)-8:

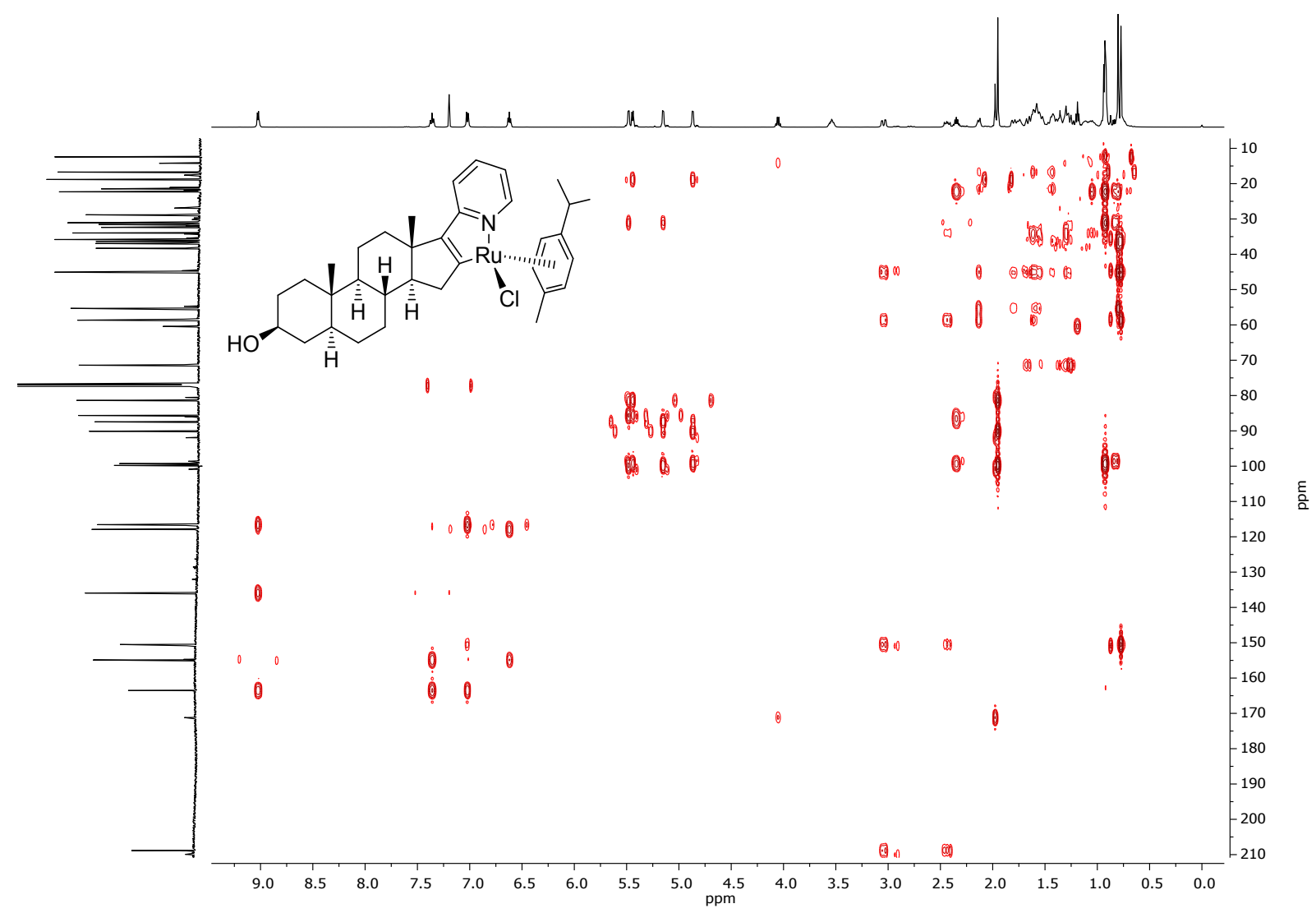


COSY spectra of Ru-complex (S-Ru)-8:
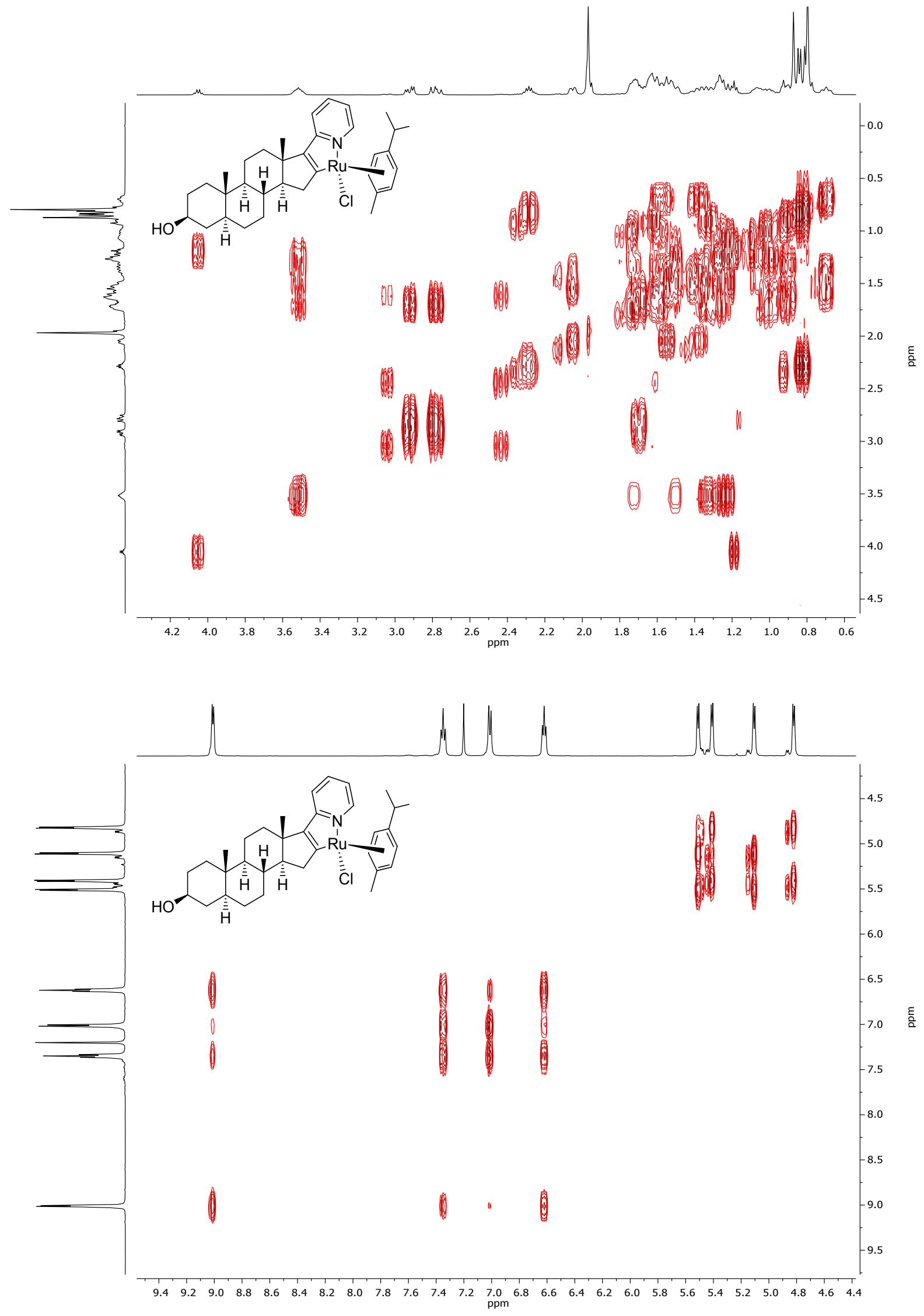
NOESY spectra of Ru-complex ( $\boldsymbol{S}$-Ru)-8:
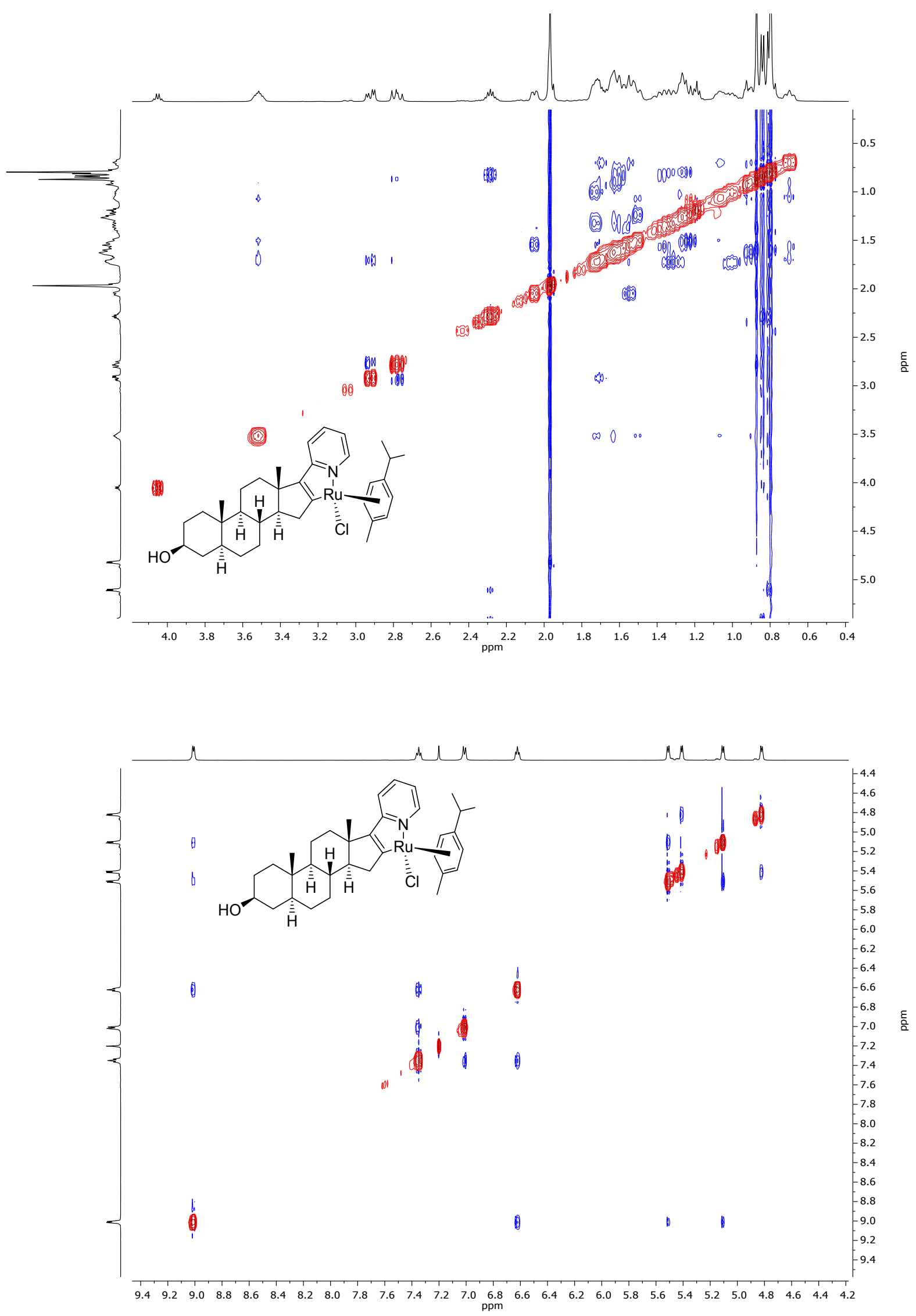
HSQC spectra of Ru-complex (S-Ru)-8:
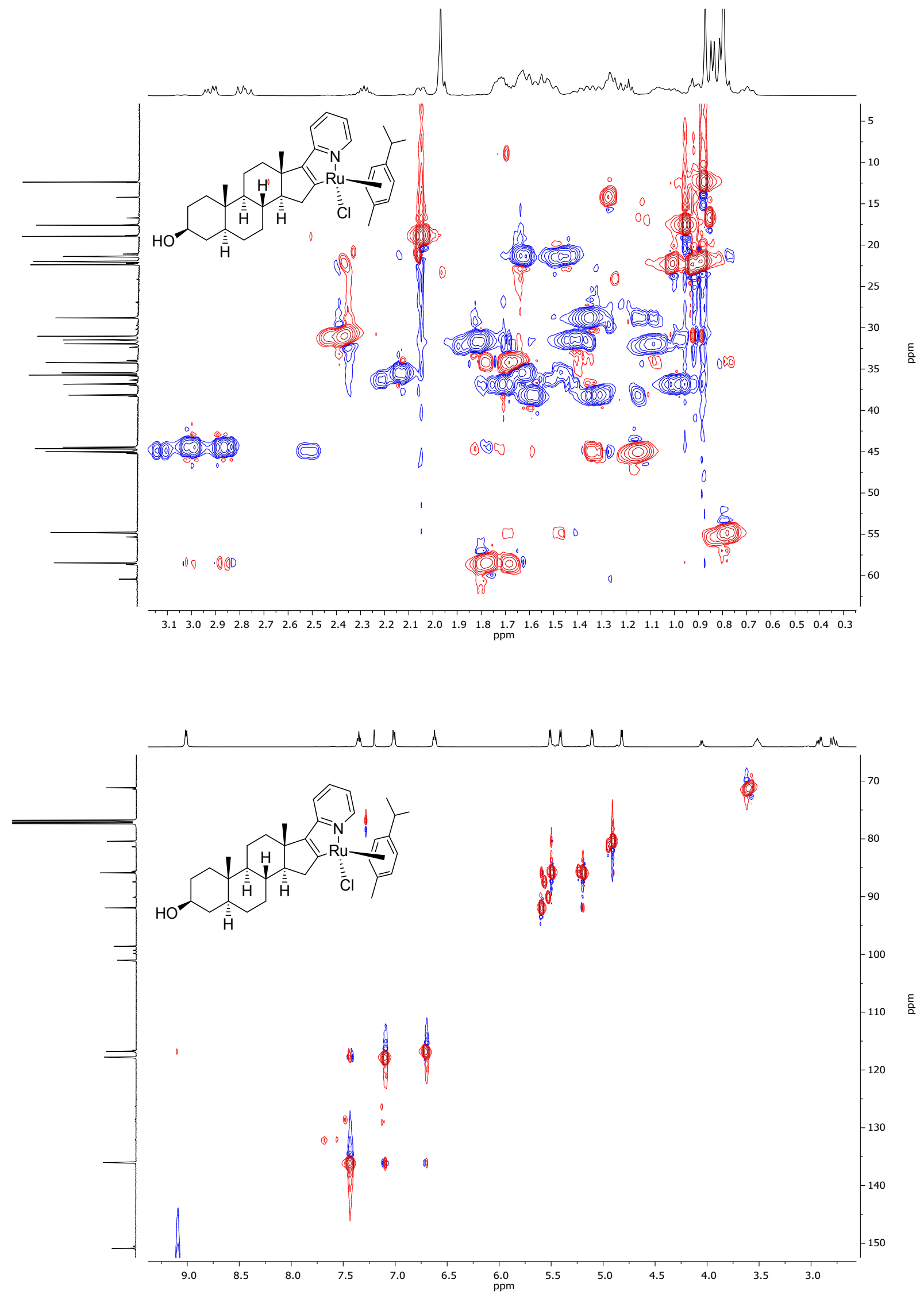
HMBC spectrum of Ru-complex ( $\boldsymbol{S}$-Ru)-8:

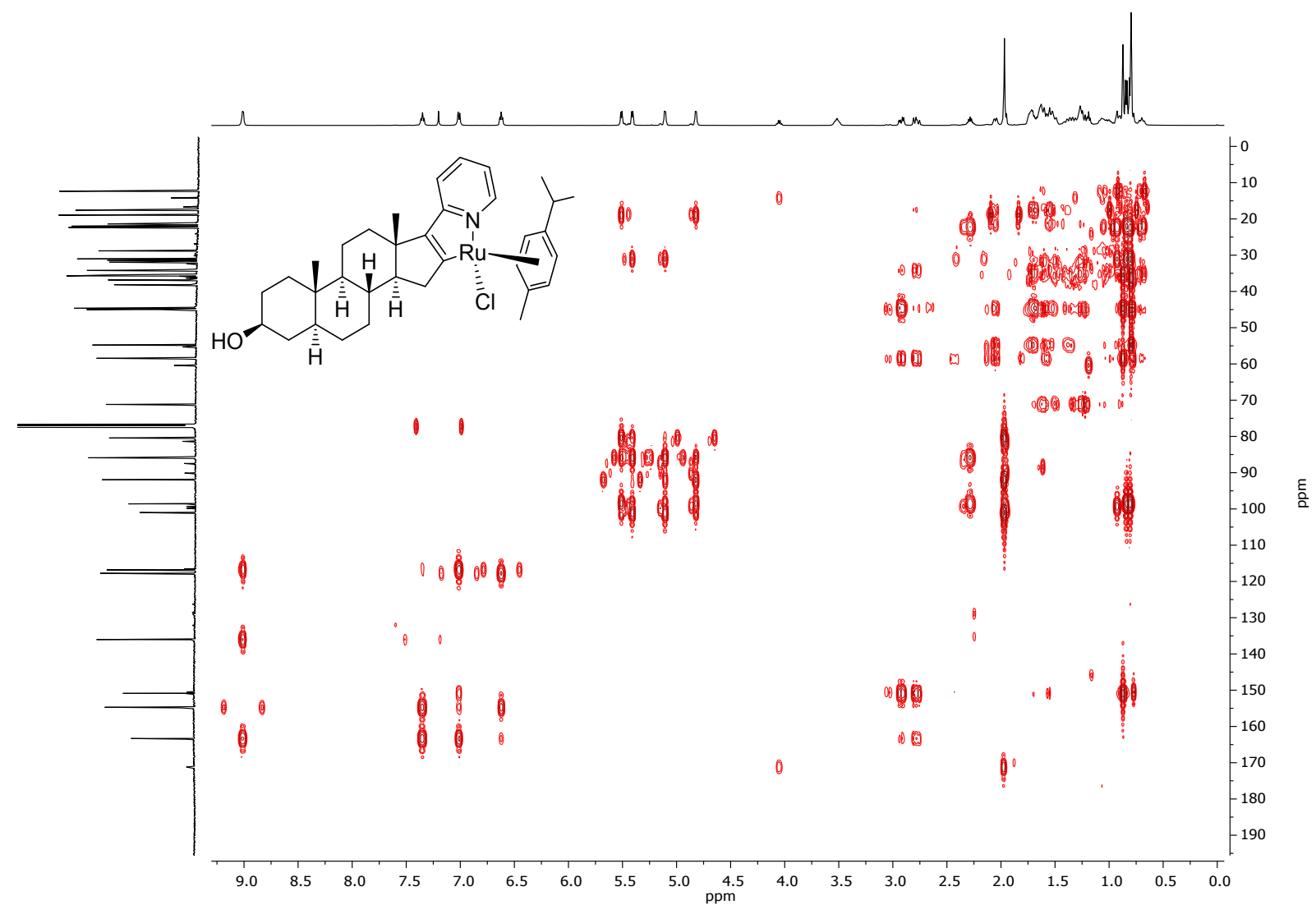




\subsection{X-ray crystallography}

The single-crystal X-ray diffraction were carried out by Dr. Martin Nieger $(\mathbf{5 b}, \mathbf{7})$ and Dr. Olaf Fuhr ((R-Ru)-8, 12 and 13).

The single-crystal X-ray diffraction study of $5 b$ [V. Koch, M. Nieger, S. Bräse, Adv. Synth. Catal. 2017, 359, 832-840 $]^{2}$ and 7 was carried out on a Bruker D8 Venture diffractometer with Photon100 detector at $123(2) \mathrm{K}$ using $\mathrm{Cu}-\mathrm{K} \alpha$ radiation $(\lambda=1.54178 \AA \AA$ ). Direct Methods $\left(\right.$ SHELXS-97) ${ }^{5}$ was used for structure solution and refinement was carried out using SHELXL2014 (full-matrix least-squares on $\left.F^{2}\right)^{6}$. Hydrogen atoms were localized by difference electron density determination and refined using a riding model $(\mathrm{H}(\mathrm{O})$ free). A semi-empirical absorption corrections were applied. The absolute configuration were determined by refinement of Parsons' x-parameter?

CCDC 1521243 (5b) and 1859054 (7) contain the supplementary crystallographic data for this paper. These data can be obtained free of charge from The Cambridge Crystallographic Data Centre via www.ccdc.cam.ac.uk/data_request/cif. 


\section{Crystal data}

\begin{tabular}{|l|l|}
\hline $\mathrm{C}_{24} \mathrm{H}_{33} \mathrm{NO}$ & $D_{\mathrm{x}}=1.214 \mathrm{Mg} \mathrm{m}^{-3}$ \\
\hline$M_{r}=351.51$ & $\mathrm{Cu} K \alpha$ radiation, $\lambda=1.54178 \AA$ \\
\hline Orthorhombic, $P 2_{1} 2_{1} 2_{1}($ no.19) & Cell parameters from 9909 reflections \\
\hline$a=6.1607(4) \AA$ & $\theta=3.8-72.2^{\circ}$ \\
\hline$b=13.4846(9) \AA$ & $\mu=0.55 \mathrm{~mm}^{-1}$ \\
\hline$c=23.1554(15) \AA$ & $T=123 \mathrm{~K}$ \\
\hline$V=1923.6(2) \AA^{3}$ & Rods, colourless \\
\hline$Z=4$ & $0.20 \times 0.06 \times 0.04 \mathrm{~mm}$ \\
\hline$F(000)=768$ & \\
\hline
\end{tabular}

\section{Data collection}

\begin{tabular}{|l|l|}
\hline $\begin{array}{l}\text { Bruker D8 VENTURE diffractometer with } \\
\text { Photon100 detector }\end{array}$ & 3801 independent reflections \\
\hline $\begin{array}{l}\text { Radiation source: INCOATEC microfocus } \\
\text { sealed tube }\end{array}$ & 3681 reflections with $I>2 \sigma(I)$ \\
\hline Detector resolution: 10.4167 pixels $\mathrm{mm}^{-1}$ & $R_{\text {int }}=0.028$ \\
\hline rotation in $\phi$ and $\omega, 1^{\circ}$, shutterless scans & $\theta_{\max }=72.2^{\circ}, \theta_{\min }=3.8^{\circ}$ \\
\hline $\begin{array}{l}\text { Absorption correction: multi-scan } \\
S A D A B S \text { (Sheldrick, } 2014)\end{array}$ & $h=-7 \rightarrow 7$ \\
\hline$T_{\min }=0.869, T_{\max }=0.971$ & $k=-16 \rightarrow 16$ \\
\hline 17442 measured reflections & $l=-28 \rightarrow 27$ \\
\hline
\end{tabular}

\section{Refinement}

\begin{tabular}{|l|l|}
\hline Refinement on $F^{2}$ & $\begin{array}{l}\text { Secondary atom site location: difference Fourier } \\
\text { map }\end{array}$ \\
\hline Least-squares matrix: full & Hydrogen site location: difference Fourier map \\
\hline$R\left[F^{2}>2 \sigma\left(F^{2}\right)\right]=0.032$ & $\begin{array}{l}\text { H atoms treated by a mixture of independent } \\
\text { and constrained refinement }\end{array}$ \\
\hline$w R\left(F^{2}\right)=0.080$ & $\begin{array}{l}w=1 /\left[\sigma^{2}\left(F_{\mathrm{o}}^{2}\right)+(0.0421 P)^{2}+0.5125 P\right] \\
\text { where } P=\left(F_{\mathrm{o}}^{2}+2 F_{\mathrm{c}}^{2}\right) / 3\end{array}$ \\
\hline$S=1.04$ & $(\Delta / \sigma)_{\max }<0.001$ \\
\hline 3801 reflections & $\Delta\rangle_{\max }=0.22$ e $\AA^{-3}$ \\
\hline 238 parameters & $\Delta\rangle_{\min }=-0.17$ e $\AA^{-3}$ \\
\hline 0 restraints & $\begin{array}{l}\text { Absolute structure: Flack x determined using } \\
1508 \text { quotients [(I+)-(I-)]/[(I+)+(I-)] (Parsons, } \\
\text { Flack and Wagner, Acta Cryst. B69 (2013) 249- } \\
259) .\end{array}$ \\
\hline $\begin{array}{l}\text { Primary atom site location: structure-invariant } \\
\text { direct methods }\end{array}$ & Absolute structure parameter: 0.04 (9) \\
\hline
\end{tabular}




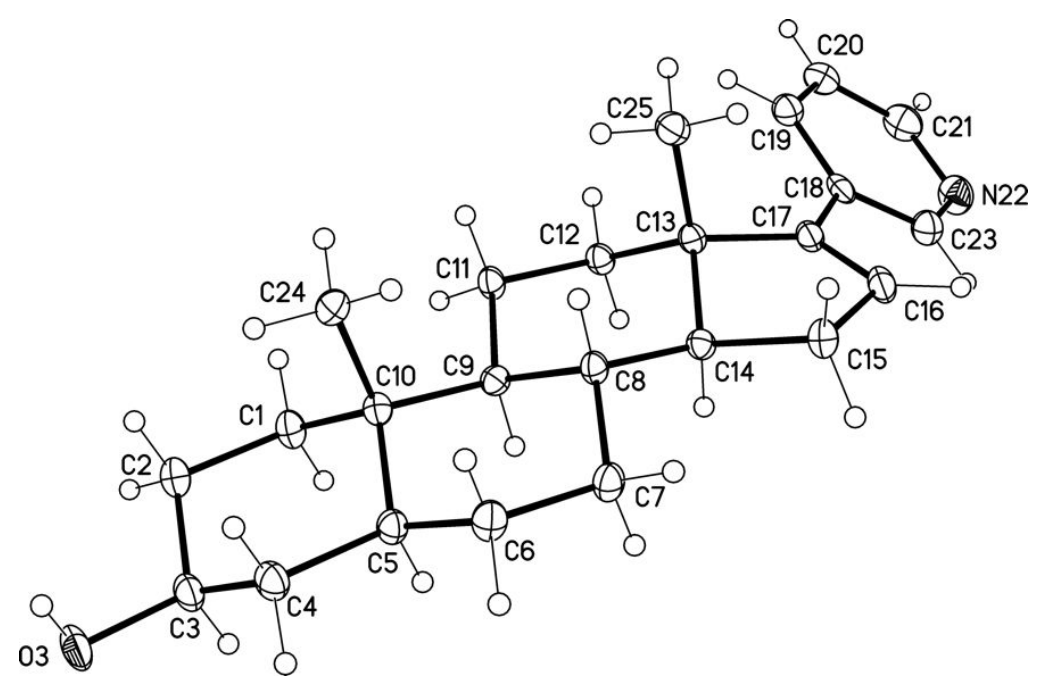

Figure 1: Molecular structure of $\mathbf{5 b}$ (displacement parameters are drawn at 50\% probability level). 
Chlorido( $\eta^{6}$-para-cymene)[17-(4'-pyridin-2 ' '-yl- $\kappa N$-phenyl-3 $\left.{ }^{\prime} \kappa C\right)-(5 \alpha, 3 \beta)$-androst-16-enolido]ruthenium(II) (7)

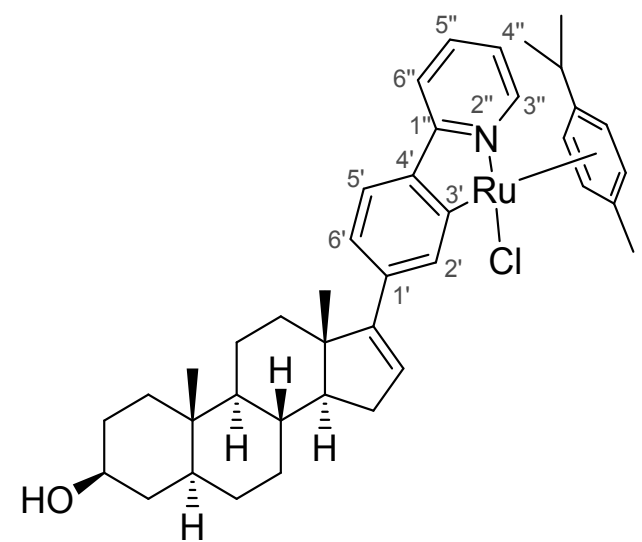

Crystal data

\begin{tabular}{|l|l|}
\hline $\mathrm{C}_{40} \mathrm{H}_{50} \mathrm{CINORu}$ & $D_{\mathrm{x}}=1.391 \mathrm{Mg} \mathrm{m}^{-3}$ \\
\hline$M_{r}=697.33$ & Cu $K \alpha$ radiation, $\lambda=1.54178 \AA$ \\
\hline Orthorhombic, $P 2_{1} 2_{1} 2_{1}$ (no.19) & Cell parameters from 9783 reflections \\
\hline$a=8.1509(2) \AA$ & $\theta=4.4-72.0^{\circ}$ \\
\hline$b=10.2164(3) \AA$ & $\mu=4.79 \mathrm{~mm}^{-1}$ \\
\hline$c=39.9845(10) \AA$ & $T=123 \mathrm{~K}$ \\
\hline$V=3329.62(15) \AA^{3}$ & Plates, orange \\
\hline$Z=4$ & $0.16 \times 0.14 \times 0.04 \mathrm{~mm}$ \\
\hline$F(000)=1464$ & \\
\hline
\end{tabular}

\section{Data collection}

\begin{tabular}{|l|l|}
\hline $\begin{array}{l}\text { Bruker D8 VENTURE diffractometer with } \\
\text { Photon100 detector }\end{array}$ & 6513 independent reflections \\
\hline $\begin{array}{l}\text { Radiation source: INCOATEC microfocus sealed } \\
\text { tube }\end{array}$ & 6455 reflections with $I>2 \sigma(I)$ \\
\hline Detector resolution: 10.4167 pixels $\mathrm{mm}^{-1}$ & $R_{\text {int }}=0.020$ \\
\hline rotation in $\phi$ and $\omega, 0.5^{\circ}$, shutterless scans & $\theta_{\max }=72.1^{\circ}, \theta_{\min }=4.4^{\circ}$ \\
\hline $\begin{array}{l}\text { Absorption correction: multi-scan } \\
S A D A B S / \text { Sheldrick, } 2014)\end{array}$ & $h=-10 \rightarrow 10$ \\
\hline$T_{\min }=0.687, T_{\max }=0.829$ & $k=-12 \rightarrow 12$ \\
\hline 22924 measured reflections & $I=-42 \rightarrow 49$ \\
\hline
\end{tabular}




\section{Refinement}

\begin{tabular}{|l|l|}
\hline Refinement on $F^{2}$ & Secondary atom site location: difference Fourier map \\
\hline Least-squares matrix: full & Hydrogen site location: difference Fourier map \\
\hline$R\left[F^{2}>2 \sigma\left(F^{2}\right)\right]=0.024$ & $\begin{array}{l}\text { H atoms treated by a mixture of independent and } \\
\text { constrained refinement }\end{array}$ \\
\hline$w R\left(F^{2}\right)=0.061$ & $\begin{array}{l}w=1 /\left[\sigma^{2}\left(F_{\mathrm{o}}{ }^{2}\right)+(0.0376 P)^{2}+1.3266 P\right] \\
\text { where } P=\left(F_{\mathrm{o}}{ }^{2}+2 F_{\mathrm{c}}{ }^{2}\right) / 3\end{array}$ \\
\hline$S=1.11$ & $(\Delta / \sigma)_{\max }=0.002$ \\
\hline 6513 reflections & $\Delta\rangle_{\max }=0.68$ e $\AA^{-3}$ \\
\hline 401 parameters & $\Delta\rangle_{\min }=-0.34$ e $\AA^{-3}$ \\
\hline 1 restraint & $\begin{array}{l}\text { Absolute structure: Flack } x \text { determined using 2704 } \\
\text { quotients }[(I+)-(I-)] /[(I+)+(I-)](\text { Parsons, Flack and Wagner, } \\
\text { Acta Cryst. B69 (2013) 249-259). }\end{array}$ \\
\hline $\begin{array}{l}\text { Primary atom site location: structure-invariant } \\
\text { direct methods }\end{array}$ & Absolute structure parameter: -0.011 (2) \\
\hline
\end{tabular}

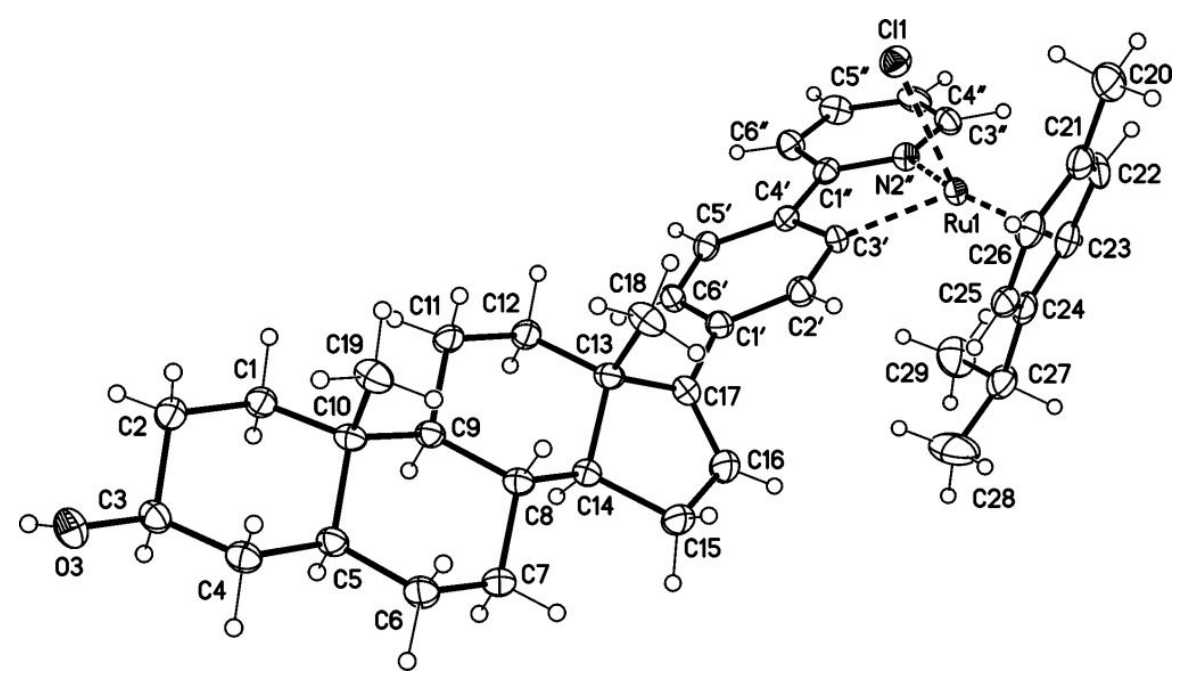

Figure 2: Molecular structure of 7 (displacement parameters are drawn at 50\% probability level). 
For single crystal X-ray analysis suitable crystals were selected and $(R)-8,12$ and $\mathbf{1 3}$ were investigated on a Stoe StadiVari diffractometer using Ga-K $\alpha$ radiation $(\lambda=1.34143 \AA)$ generated by an Metaljet Xray source. The crystals were kept at $180.15 \mathrm{~K}$ during data collection. Using Olex $2^{8}$, the structures were solved with the ShelXS ${ }^{5}$ structure solution program using Direct Methods and refined with the ShelXL ${ }^{6}$ refinement package using Least Squares minimization. Non-hydrogen atoms were refined with anisotropic displacement parameters; hydrogen atoms were modelled on idealized positions. 


\section{$\underline{\text { ruthenium(II) }((\boldsymbol{R}-\mathbf{R u}) \underline{-8})}$}

\begin{tabular}{|c|c|}
\hline Empirical formula & $\mathrm{C}_{34} \mathrm{H}_{46} \mathrm{ClNORu}$ \\
\hline Formula weight & 621.24 \\
\hline Temperature/K & 180.15 \\
\hline Crystal system & hexagonal \\
\hline Space group & $\mathrm{P} 6_{5}$ \\
\hline $\mathrm{a} / \AA$ & $31.5730(9)$ \\
\hline $\mathrm{b} / \AA$ & $31.5730(9)$ \\
\hline $\mathrm{c} / \AA$ & $10.3106(2)$ \\
\hline$\alpha /{ }^{\circ}$ & 90 \\
\hline$\beta /{ }^{\circ}$ & 90 \\
\hline$\gamma /{ }^{\circ}$ & 120 \\
\hline Volume $/ \AA^{3}$ & $8901.1(5)$ \\
\hline $\mathrm{Z}$ & 6 \\
\hline$\rho_{\text {calc }} \mathrm{g} / \mathrm{cm}^{3}$ & 0.695 \\
\hline$\mu / \mathrm{mm}^{-1}$ & 1.788 \\
\hline $\mathrm{F}(000)$ & 1956.0 \\
\hline Crystal size $/ \mathrm{mm}^{3}$ & $0.27 \times 0.05 \times 0.04$ \\
\hline Radiation & $\operatorname{GaK} \alpha(\lambda=1.34143)$ \\
\hline $2 \Theta$ range for data collection $/{ }^{\circ}$ & 7.442 to 118.8 \\
\hline Index ranges & $-40 \leq \mathrm{h} \leq 39,-37 \leq \mathrm{k} \leq 40,-12 \leq 1 \leq 6$ \\
\hline Reflections collected & 50192 \\
\hline Independent reflections & $8931\left[\mathrm{R}_{\mathrm{int}}=0.0829, \mathrm{R}_{\text {sigma }}=0.0764\right]$ \\
\hline Indep. refl. with $\mathrm{I}>=2 \sigma(\mathrm{I})$ & 3717 \\
\hline Data/restraints/parameters & $8931 / 1 / 350$ \\
\hline Goodness-of-fit on $\mathrm{F}^{2}$ & 0.803 \\
\hline Final $R$ indexes $[\mathrm{I}>=2 \sigma(\mathrm{I})]$ & $\mathrm{R}_{1}=0.0940, \mathrm{wR}_{2}=0.2189$ \\
\hline Final $\mathrm{R}$ indexes [all data] & $\mathrm{R}_{1}=0.1274, \mathrm{w} \mathrm{R}_{2}=0.2277$ \\
\hline Largest diff. peak/hole / e $\AA^{-3}$ & $0.77 /-0.44$ \\
\hline Flack parameter & $0.05(2)$ \\
\hline
\end{tabular}


Dichlorido( $\eta^{6}$-para-cymene $)\left[(5 \alpha, 3 \beta)-17-\left(\left(3^{1}\right.\right.\right.$-pyridinyl- $\left.\kappa \mathrm{N}\right)$-androst-16-en-3-ol)ido]ruthenium(II) (12)

\begin{tabular}{|c|c|}
\hline Empirical formula & $\mathrm{C}_{34} \mathrm{H}_{47} \mathrm{Cl}_{2} \mathrm{NORu}$ \\
\hline Formula weight & 657.69 \\
\hline Temperature/K & 180.15 \\
\hline Crystal system & orthorhombic \\
\hline Space group & $\mathrm{P} 2{ }_{1} 2_{1} 2_{1}$ \\
\hline $\mathrm{a} / \AA$ & $7.0251(2)$ \\
\hline $\mathrm{b} / \AA$ & $15.6281(5)$ \\
\hline $\mathrm{c} / \AA$ & $28.6301(12)$ \\
\hline$\alpha /^{\circ}$ & 90 \\
\hline$\beta /{ }^{\circ}$ & 90 \\
\hline$\gamma /{ }^{\circ}$ & 90 \\
\hline Volume $/ \AA^{3}$ & $3143.27(19)$ \\
\hline $\mathrm{Z}$ & 4 \\
\hline$\rho_{\text {calc }} \mathrm{g} / \mathrm{cm}^{3}$ & 1.390 \\
\hline$\mu / \mathrm{mm}^{-1}$ & 3.844 \\
\hline $\mathrm{F}(000)$ & 1376.0 \\
\hline Crystal size $/ \mathrm{mm}^{3}$ & $0.1 \times 0.08 \times 0.03$ \\
\hline Radiation & $\operatorname{GaK} \alpha(\lambda=1.34143)$ \\
\hline $2 \Theta$ range for data collection $/{ }^{\circ}$ & 11.832 to 118.208 \\
\hline Index ranges & $-8 \leq \mathrm{h} \leq 8,-19 \leq \mathrm{k} \leq 8,-35 \leq 1 \leq 35$ \\
\hline Reflections collected & 23083 \\
\hline Independent reflections & $6440\left[\mathrm{R}_{\mathrm{int}}=0.0255, \mathrm{R}_{\mathrm{sigma}}=0.0339\right]$ \\
\hline Indep. refl. with $\mathrm{I}>=2 \sigma$ (I) & 5586 \\
\hline Data/restraints/parameters & $6440 / 0 / 352$ \\
\hline Goodness-of-fit on $\mathrm{F}^{2}$ & 1.045 \\
\hline Final $\mathrm{R}$ indexes $[\mathrm{I}>=2 \sigma(\mathrm{I})]$ & $\mathrm{R}_{1}=0.0342, \mathrm{wR}_{2}=0.0832$ \\
\hline Final $\mathrm{R}$ indexes [all data] & $\mathrm{R}_{1}=0.0397, \mathrm{wR}_{2}=0.0848$ \\
\hline Largest diff. peak/hole / e $\AA^{-3}$ & $0.47 /-0.52$ \\
\hline Flack parameter & $-0.035(7)$ \\
\hline
\end{tabular}


Dichlorido $\left(\eta^{5}-\right.$ pentamethylcyclopentadienyl $)\left[(5 \alpha, 3 \beta)-17-\left(\left(3^{\prime}-\right.\right.\right.$ pyridinyl- $\left.\kappa \mathrm{N}\right)$ androst-16-en-3-ol)ido $]-$

iridium(III) (13)

\begin{tabular}{|c|c|}
\hline Empirical formula & $\mathrm{C}_{34} \mathrm{H}_{48} \mathrm{Cl}_{2} \mathrm{IrNO}$ \\
\hline Formula weight & 749.83 \\
\hline Temperature/K & 180.15 \\
\hline Crystal system & orthorhombic \\
\hline Space group & $\mathrm{P} 2{ }_{1} 2_{1} 2_{1}$ \\
\hline $\mathrm{a} / \AA$ & $6.9313(2)$ \\
\hline $\mathrm{b} / \AA$ & $17.0346(7)$ \\
\hline $\mathrm{c} / \AA$ & $32.1445(13)$ \\
\hline$\alpha /^{\circ}$ & 90 \\
\hline$\beta /{ }^{\circ}$ & 90 \\
\hline$\gamma /{ }^{\circ}$ & 90 \\
\hline Volume $/ \AA^{3}$ & $3795.4(2)$ \\
\hline $\mathrm{Z}$ & 4 \\
\hline$\rho_{\text {calc }} \mathrm{g} / \mathrm{cm}^{3}$ & 1.312 \\
\hline$\mu / \mathrm{mm}^{-1}$ & 5.488 \\
\hline $\mathrm{F}(000)$ & 1512.0 \\
\hline Crystal size $/ \mathrm{mm}^{3}$ & $0.24 \times 0.2 \times 0.02$ \\
\hline Radiation & $\operatorname{GaK} \alpha(\lambda=1.34143)$ \\
\hline $2 \Theta$ range for data collection ${ }^{\circ}$ & 6.578 to 105.986 \\
\hline Index ranges & $-8 \leq \mathrm{h} \leq 4,-20 \leq \mathrm{k} \leq 20,-38 \leq 1 \leq 22$ \\
\hline Reflections collected & 11444 \\
\hline Independent reflections & $6364\left[\mathrm{R}_{\mathrm{int}}=0.0569, \mathrm{R}_{\text {sigma }}=0.0594\right]$ \\
\hline Indep. refl. with $\mathrm{I}>=2 \sigma(\mathrm{I})$ & 5200 \\
\hline Data/restraints/parameters & $6364 / 60 / 307$ \\
\hline Goodness-of-fit on $\mathrm{F}^{2}$ & 1.099 \\
\hline Final $\mathrm{R}$ indexes $[\mathrm{I}>=2 \sigma(\mathrm{I})]$ & $\mathrm{R}_{1}=0.0922, \mathrm{wR}_{2}=0.2578$ \\
\hline Final $\mathrm{R}$ indexes [all data] & $\mathrm{R}_{1}=0.1020, \mathrm{wR}_{2}=0.2639$ \\
\hline Largest diff. peak/hole / e $\AA^{-3}$ & $2.67 /-2.79$ \\
\hline Flack parameter & $0.18(5)$ \\
\hline
\end{tabular}




\section{Quantum Chemical Calculations}

\subsection{Calculated Chemical Shifts}

Table S1: Selected chemical Shift differences $\Delta_{\delta}=\delta_{\mathrm{S}}-\delta_{\mathrm{R}}$ of diastereomer $(\boldsymbol{R}-\mathbf{R u})-\mathbf{8}$ and $(\boldsymbol{S}-\mathbf{R u})-\mathbf{8}$ calculated in the def2-TZVPP 9 level of theory applying different exchange-correlation functionals. All functionals reproduce the experimental observed trends in chemical shift differences of the two diastereomers except for the ${ }^{13} \mathrm{C}$ shift of carbon 14. The shift difference of Carbon 15 is not correctly predicted by the M06 and M06-2X functionals ${ }^{10}$. The hybrid functionals including exact Hartree-Fock exchange (B3LYP ${ }^{11}, \mathrm{TPSSh}^{12}$ ) are closer to the experimental ${ }^{13} \mathrm{C}$ shifts than their GGA (bp86 ${ }^{13-14}$ ) counterpart. However, in case of ${ }^{1} \mathrm{H}$ chemical shift differences BP86 performs equally well like TPSSh. Interestingly, the meta-GGA hybrids M06 (27\% HF-exchange) and M06-2X (54\% Hartree-Fock exchange) compare worse to the experimental values for both ${ }^{1} \mathrm{H}$ and ${ }^{13} \mathrm{C}$ chemical shifts. Nevertheless, one should keep in mind that the chemical shift differences of the present study are very small compared to the general accuracy of DFT chemical sift predictions. In addition, for all NMR shift predictions the bp86/def2-TZVPP structures were used throughout. A more thorough comparison would require optimizations using all functionals discussed. Hence, the presented discussion should not be interpreted as a general functional benchmark for NMR shift calculations. MAE: Mean Absolute Error.

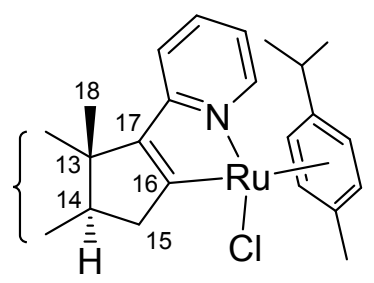

8

\begin{tabular}{|c|c|c|c|c|c|c|c|}
\hline & & B3LYP & BP86 & M06 & M06-2X & TPSSh & Exp. \\
\hline \multirow{6}{*}{$\mathbf{C}$} & $13-\mathrm{C}_{\mathrm{q}}$ & -0.16 & -0.12 & -0.36 & -0.21 & -0.15 & -0.50 \\
\hline & 14-CH & 0.90 & 1.05 & 0.63 & 0.57 & 1.09 & -0.20 \\
\hline & $15-\mathrm{CH}_{2}$ & -0.09 & -0.23 & 0.34 & 0.02 & -0.30 & -0.50 \\
\hline & $16-\mathrm{C}_{\mathrm{q}}-\mathrm{Ru}$ & 0.71 & 0.49 & 1.29 & 0.21 & 0.59 & 1.00 \\
\hline & $17-\mathrm{C}_{\mathrm{q}}-\mathrm{Pyr}$ & 0.30 & 0.20 & 1.11 & 0.79 & 0.15 & 0.30 \\
\hline & $18-\mathrm{CH}_{3}$ & 1.85 & 1.72 & 2.15 & 2.31 & 1.66 & 0.80 \\
\hline \multirow[t]{2}{*}{$M A E$} & & 0.53 & 0.57 & 0.71 & 0.73 & 0.54 & -- \\
\hline & 14-CH & -0.04 & 0.00 & -0.19 & -0.18 & -0.01 & n.d. \\
\hline \multirow{3}{*}{ H } & $15 \alpha-\mathrm{CH}_{2}$ & -0.29 & -0.28 & -0.39 & -0.41 & -0.25 & -0.13 \\
\hline & $15 \beta-\mathrm{CH}_{2}$ & 0.36 & 0.34 & 0.42 & 0.56 & 0.33 & 0.34 \\
\hline & $18-\mathrm{CH}_{3}$ & 0.13 & 0.12 & 0.16 & 0.24 & 0.12 & 0.09 \\
\hline$M A E$ & & 0.08 & 0.06 & 0.14 & 0.22 & 0.06 & -- \\
\hline
\end{tabular}


Table S2: Selected chemical Shift differences $\Delta_{\delta}=\delta_{\mathrm{S}}-\delta_{\mathrm{R}}$ of diastereomer $(\boldsymbol{R})-\mathbf{7}$ and $(\boldsymbol{S})-\mathbf{7}$ calculated in the def2-TZVPP basis using different exchange-correlation functionals.

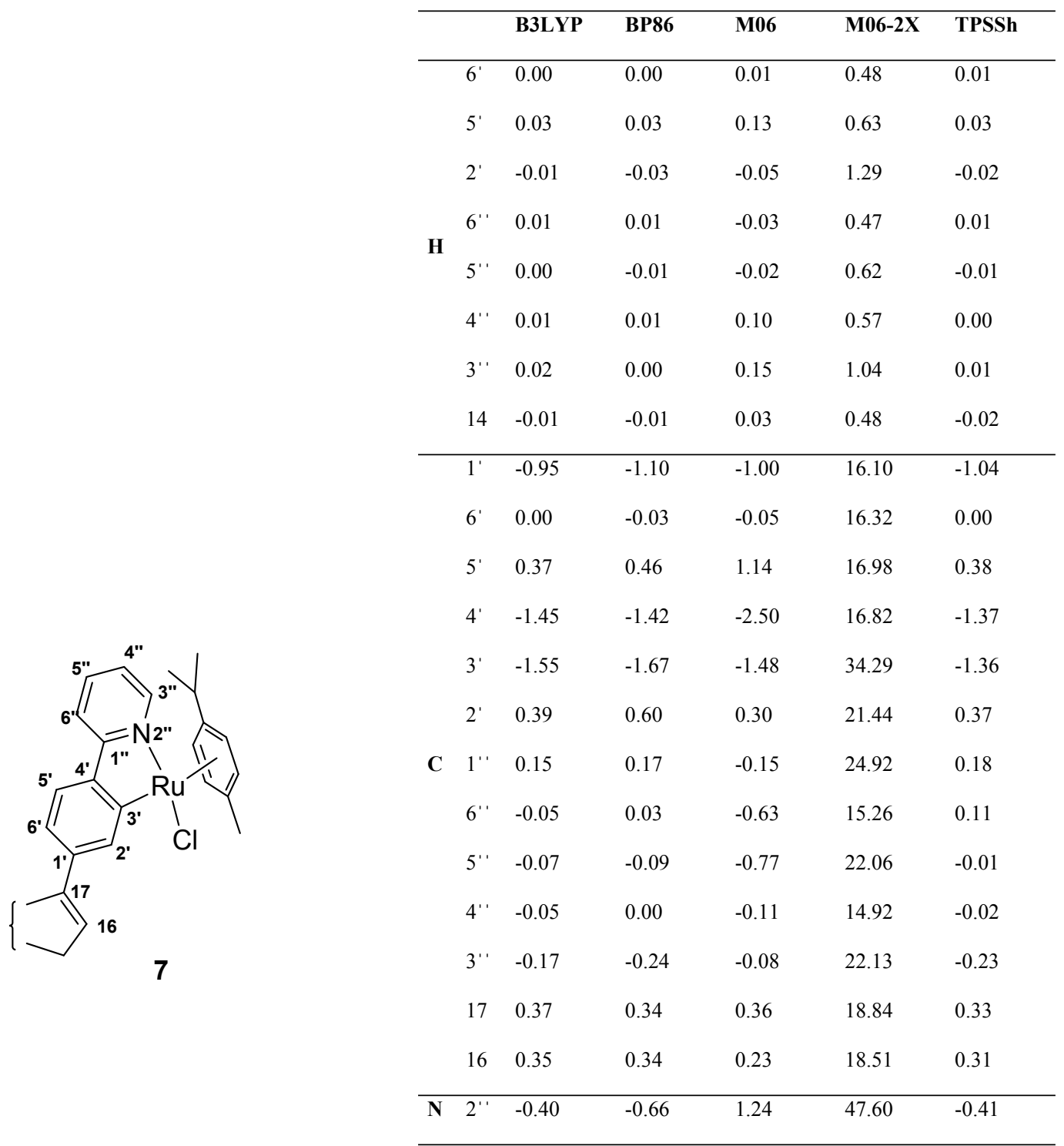




\subsection{Computational Studies}

XYZ-Coordinates (Ångström) of optimized structures (bp86/def2-TZVPP).

\section{( $R-\mathbf{R u})-7$}

\begin{tabular}{|c|c|c|c|}
\hline C & -3.569295 & 0.641060 & -4.861566 \\
\hline $\mathrm{H}$ & -4.276180 & 0.280617 & -4.101449 \\
\hline $\mathrm{H}$ & -3.185688 & 1.610888 & -4.500056 \\
\hline $\mathrm{C}$ & -4.321272 & 0.856599 & -6.181824 \\
\hline $\mathrm{H}$ & -4.802104 & -0.077740 & -6.510462 \\
\hline $\mathrm{H}$ & -5.126777 & 1.594016 & -6.035227 \\
\hline C & -3.384292 & 1.329811 & -7.290799 \\
\hline $\mathrm{H}$ & -2.987457 & 2.327490 & -7.017775 \\
\hline O & -4.057222 & 1.421355 & -8.563710 \\
\hline $\mathrm{H}$ & -4.814791 & 2.019600 & -8.452000 \\
\hline C & -2.209578 & 0.376361 & -7.454556 \\
\hline $\mathrm{H}$ & -1.520926 & 0.770857 & -8.217081 \\
\hline $\mathrm{H}$ & -2.581736 & -0.589453 & -7.831744 \\
\hline C & -1.460622 & 0.193903 & -6.129535 \\
\hline $\mathrm{H}$ & -1.139778 & 1.202336 & -5.803055 \\
\hline C & -0.187168 & -0.639045 & -6.289373 \\
\hline $\mathrm{H}$ & -0.446044 & -1.664172 & -6.600114 \\
\hline $\mathrm{H}$ & 0.425349 & -0.216167 & -7.100877 \\
\hline C & 0.612598 & -0.670583 & -4.986473 \\
\hline $\mathrm{H}$ & 1.501510 & -1.309790 & -5.094680 \\
\hline $\mathrm{H}$ & 0.981968 & 0.347088 & -4.766029 \\
\hline C & -0.230692 & -1.150011 & -3.800460 \\
\hline $\mathrm{H}$ & -0.489187 & -2.207773 & -3.972717 \\
\hline C & -1.535515 & -0.318366 & -3.673469 \\
\hline $\mathrm{H}$ & -1.203700 & 0.731644 & -3.547351 \\
\hline C & -2.377037 & -0.335853 & -4.986229 \\
\hline C & -2.343479 & -0.681166 & -2.409948 \\
\hline $\mathrm{H}$ & -3.168957 & 0.033405 & -2.288788 \\
\hline $\mathrm{H}$ & -2.815325 & -1.665441 & -2.544404 \\
\hline C & -1.518327 & -0.695532 & -1.108908 \\
\hline $\mathrm{H}$ & -1.192867 & 0.327947 & -0.861597 \\
\hline $\mathrm{H}$ & -2.155963 & -1.032749 & -0.279115 \\
\hline C & -0.274641 & -1.577898 & -1.266761 \\
\hline C & 0.520498 & -1.029184 & -2.481993 \\
\hline $\mathrm{H}$ & 0.627489 & 0.054983 & -2.285010 \\
\hline C & 1.932798 & -1.605619 & -2.289908 \\
\hline $\mathrm{H}$ & 2.026798 & -2.631416 & -2.691559 \\
\hline $\mathrm{H}$ & 2.716236 & -1.007090 & -2.776367 \\
\hline $\mathrm{C}$ & 2.031955 & -1.589981 & -0.783356 \\
\hline $\mathrm{Ru}$ & 2.567795 & 0.384927 & 4.728719 \\
\hline $\mathrm{C}$ & 0.818440 & -1.503493 & -0.187889 \\
\hline C & 0.121004 & -1.095854 & 5.479725 \\
\hline C & -0.867056 & -1.645978 & 6.310357 \\
\hline $\mathrm{H}$ & -1.691937 & -2.197578 & 5.863339 \\
\hline C & -0.780043 & -1.497487 & 7.688354 \\
\hline $\mathrm{H}$ & -1.544922 & -1.922213 & 8.337469 \\
\hline C & 0.312443 & -0.808728 & 8.229667 \\
\hline C & 1.263215 & -0.282002 & 7.366458 \\
\hline $\mathrm{N}$ & 1.166012 & -0.396659 & 6.025588 \\
\hline C & -2.904461 & -1.751572 & -5.283299 \\
\hline $\mathrm{H}$ & -2.099985 & -2.495798 & -5.312221 \\
\hline $\mathrm{H}$ & -3.621161 & -2.065868 & -4.512954 \\
\hline $\mathrm{H}$ & -3.425129 & -1.797840 & -6.247245 \\
\hline C & -0.666606 & -3.063165 & -1.450659 \\
\hline $\mathrm{H}$ & -1.391051 & -3.202807 & -2.262103 \\
\hline $\mathrm{H}$ & 0.215377 & -3.676385 & -1.676571 \\
\hline $\mathrm{H}$ & -1.118503 & -3.461487 & -0.533331 \\
\hline $\mathrm{Cl}$ & 3.779939 & -1.664820 & 5.083430 \\
\hline $\mathrm{H}$ & 2.137362 & 0.249570 & 7.734878 \\
\hline $\mathrm{H}$ & 0.433166 & -0.682448 & 9.303612 \\
\hline C & 2.086614 & 2.427262 & 5.237095 \\
\hline C & 2.045705 & 2.292895 & 3.817824 \\
\hline C & 3.231922 & 1.786386 & 3.183824 \\
\hline C & 4.357674 & 1.413977 & 3.946603 \\
\hline C & 4.424147 & 1.658695 & 5.370739 \\
\hline C & 3.288655 & 2.190237 & 5.992247 \\
\hline $\mathrm{C}$ & 0.835986 & 2.643579 & 2.980622 \\
\hline
\end{tabular}




$$
\begin{array}{r}
3.226112 \\
5.193705 \\
5.637294 \\
3.281047 \\
5.828056 \\
6.524052 \\
5.504451 \\
-0.491502 \\
0.837602 \\
0.991224 \\
1.188053 \\
-0.583854 \\
-0.593519 \\
-1.329011 \\
0.157966 \\
0.995959 \\
1.928860 \\
2.978021 \\
0.583836 \\
1.536375 \\
-0.585118 \\
-0.765017 \\
0.198011 \\
1.363063 \\
-1.341444 \\
-1.662758 \\
2.423356
\end{array}
$$

\begin{tabular}{|c|c|c|c|}
\hline $\mathrm{Ru}$ & -3.513723 & 0.354745 & 0.407982 \\
\hline $\mathrm{Cl}$ & -3.492517 & -0.562880 & 2.636610 \\
\hline O & 8.616396 & 1.160843 & -1.330255 \\
\hline $\mathrm{H}$ & 8.767100 & 2.119486 & -1.280117 \\
\hline $\mathrm{N}$ & -3.432267 & -1.623488 & -0.216453 \\
\hline C & 5.455362 & -0.880527 & -1.519345 \\
\hline $\mathrm{H}$ & 5.331140 & -1.972192 & -1.520729 \\
\hline $\mathrm{H}$ & 5.032642 & -0.514799 & -2.471271 \\
\hline C & 6.953067 & -0.552020 & -1.474614 \\
\hline $\mathrm{H}$ & 7.421054 & -1.001658 & -0.585042 \\
\hline $\mathrm{H}$ & 7.460581 & -0.982075 & -2.350424 \\
\hline $\mathrm{C}$ & 7.192961 & 0.950181 & -1.434512 \\
\hline $\mathrm{H}$ & 6.829545 & 1.390998 & -2.383774 \\
\hline C & 6.427756 & 1.594198 & -0.280548 \\
\hline $\mathrm{H}$ & 6.569564 & 2.687513 & -0.305136 \\
\hline $\mathrm{H}$ & 6.851795 & 1.239445 & 0.672081 \\
\hline C & 4.930357 & 1.275789 & -0.366639 \\
\hline $\mathrm{H}$ & 4.585069 & 1.632436 & -1.356717 \\
\hline C & 4.107700 & 2.028634 & 0.680983 \\
\hline $\mathrm{H}$ & 4.400325 & 1.703166 & 1.692401 \\
\hline $\mathrm{H}$ & 4.339948 & 3.103682 & 0.623673 \\
\hline C & 2.610759 & 1.800118 & 0.468224 \\
\hline $\mathrm{H}$ & 2.309325 & 2.259305 & -0.490603 \\
\hline $\mathrm{H}$ & 2.027654 & 2.303560 & 1.253742 \\
\hline C & 2.250291 & 0.311535 & 0.428390 \\
\hline $\mathrm{H}$ & 2.434555 & -0.111553 & 1.429724 \\
\hline C & 3.118712 & -0.442662 & -0.614765 \\
\hline $\mathrm{H}$ & 2.916343 & 0.058767 & -1.582293 \\
\hline C & 4.645812 & -0.255789 & -0.359161 \\
\hline C & 2.691536 & -1.915976 & -0.783195 \\
\hline $\mathrm{H}$ & 3.230762 & -2.355374 & -1.633389 \\
\hline $\mathrm{H}$ & 3.001707 & -2.492227 & 0.100571 \\
\hline C & 1.180044 & -2.120031 & -1.004624 \\
\hline $\mathrm{H}$ & 0.967484 & -3.199905 & -1.030062 \\
\hline $\mathrm{H}$ & 0.885557 & -1.711592 & -1.985089 \\
\hline C & 0.371318 & -1.409525 & 0.087735 \\
\hline C & 0.791505 & 0.088602 & 0.056085 \\
\hline $\mathrm{H}$ & 0.695096 & 0.384480 & -1.006718 \\
\hline C & -0.357903 & 0.825492 & 0.771013 \\
\hline $\mathrm{H}$ & -0.433086 & 1.885824 & 0.492575 \\
\hline $\mathrm{H}$ & -0.264621 & 0.796968 & 1.872029 \\
\hline C & -1.550526 & -0.000782 & 0.350569 \\
\hline C & -1.131818 & -1.238010 & -0.076706 \\
\hline
\end{tabular}

\section{(S-Ru)-7}




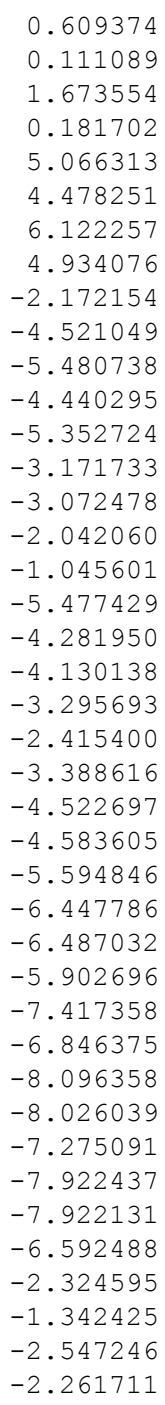

( $R$-Ru)-8

\begin{tabular}{lr} 
Ru & -3.513723 \\
Cl & -3.492517 \\
$\mathrm{O}$ & 8.616396 \\
$\mathrm{H}$ & 8.767100 \\
$\mathrm{~N}$ & -3.432267 \\
$\mathrm{C}$ & 5.455362 \\
$\mathrm{H}$ & 5.331140 \\
$\mathrm{H}$ & 5.032642 \\
$\mathrm{C}$ & 6.953067 \\
$\mathrm{H}$ & 7.421054 \\
$\mathrm{H}$ & 7.460581 \\
$\mathrm{C}$ & 7.192961 \\
$\mathrm{H}$ & 6.829545 \\
$\mathrm{C}$ & 6.427756 \\
$\mathrm{H}$ & 6.569564 \\
$\mathrm{H}$ & 6.851795 \\
$\mathrm{C}$ & 4.930357 \\
$\mathrm{H}$ & 4.585069 \\
$\mathrm{C}$ & 4.107700 \\
$\mathrm{H}$ & 4.400325 \\
$\mathrm{H}$ & 4.339948 \\
$\mathrm{C}$ & 2.610759 \\
$\mathrm{H}$ & 2.309325 \\
$\mathrm{H}$ & 2.027654 \\
$\mathrm{C}$ & 2.250291 \\
$\mathrm{H}$ & 2.434555 \\
$\mathrm{C}$ & 3.118712 \\
$\mathrm{H}$ & 2.916343 \\
$\mathrm{C}$ & 4.645812 \\
& \\
\hline
\end{tabular}

$\begin{array}{rr}-2.073652 & 1.464118 \\ -1.506996 & 2.261300 \\ -2.160558 & 1.716887 \\ -3.086162 & 1.463935 \\ -0.910424 & 0.969247 \\ -0.541607 & 1.817934 \\ -0.723761 & 1.198002 \\ -1.999506 & 0.921297 \\ -2.175765 & -0.349648 \\ -2.397612 & -0.383250 \\ -1.907033 & -0.233798 \\ -3.742105 & -0.722583 \\ -4.318964 & -0.858212 \\ -4.320038 & -0.876147 \\ -5.373766 & -1.135954 \\ -3.537631 & -0.683581 \\ -3.963611 & -0.778734 \\ 1.523272 & 0.851863 \\ 2.289285 & 1.129996 \\ 2.675391 & 2.136750 \\ 2.523007 & 0.149057 \\ 3.111760 & 0.400971 \\ 1.891200 & -1.136903 \\ 1.054267 & -1.366492 \\ 0.499733 & -2.302599 \\ 0.937335 & -0.413435 \\ 0.305784 & -0.652248 \\ 1.331822 & 1.959506 \\ 1.287886 & 2.892479 \\ 2.556405 & 2.029619 \\ 3.488981 & 2.134604 \\ 2.472788 & 2.889149 \\ 2.631724 & 1.116915 \\ 0.025844 & 1.848817 \\ 0.012613 & 0.960085 \\ -0.097513 & 2.727221 \\ -0.833121 & 1.803311 \\ 2.071506 & -2.178832 \\ -1.190370 & -1.716832 \\ -2.89178 & -2.807373 \\ -1.828644\end{array}$

$0.354745 \quad 0.407982$

$-0.562880$

1. 160843

2.119486

$-1.623488$

$-0.880527$

$-1.972192$

$-0.514799$

$-0.552020$

$-1.001658$

$-0.982075$

0.950181

1. 390998

1.594198

2. 687513

1.239445

1. 275789

1.632436

2.028634

1.703166

3. 103682

1. 800118

2. 259305

2.303560

0.311535

$-0.111553$

$-0.442662$

0.058767

$-0.255789$
2. 636610

$-1.330255$

$-1.280117$

$-0.216453$

$-1.519345$

$-1.520729$

$-2.471271$

$-1.474614$

$-0.585042$

$-2.350424$

$-1.434512$

$-2.383774$

$-0.280548$

$-0.305136$

0.672081

$-0.366639$

$-1.356717$

0.680983

1. 692401

0.623673

0.468224

$-0.490603$

1.253742

0.428390

1.429724

$-0.614765$

$-1.582293$

$-0.359161$ 


\begin{tabular}{|c|c|c|}
\hline 2.691536 & -1.915976 & -0.783195 \\
\hline 3.230762 & -2.355374 & -1.633389 \\
\hline 3.001707 & -2.492227 & 0.100571 \\
\hline 1.180044 & -2.120031 & -1.004624 \\
\hline 0.967484 & -3.199905 & -1.030062 \\
\hline 0.885557 & -1.711592 & -1.985089 \\
\hline 0.371318 & -1.409525 & 0.087735 \\
\hline 0.791505 & 0.088602 & 0.056085 \\
\hline 0.695096 & 0.384480 & -1.006718 \\
\hline-0.357903 & 0.825492 & 0.771013 \\
\hline-0.433086 & 1.885824 & 0.492575 \\
\hline-0.264621 & 0.796968 & 1.872029 \\
\hline-1.550526 & -0.000782 & 0.350569 \\
\hline-1.131818 & -1.238010 & -0.076706 \\
\hline 0.609374 & -2.073652 & 1.464118 \\
\hline 0.111089 & -1.506996 & 2.261300 \\
\hline 1.673554 & -2.160558 & 1.716887 \\
\hline 0.181702 & -3.086162 & 1.463935 \\
\hline 5.066313 & -0.910424 & 0.969247 \\
\hline 4.478251 & -0.541607 & 1.817934 \\
\hline 6.122257 & -0.723761 & 1.198002 \\
\hline 4.934076 & -1.999506 & 0.921297 \\
\hline-2.172154 & -2.175765 & -0.349648 \\
\hline-4.521049 & -2.397612 & -0.383250 \\
\hline-5.480738 & -1.907033 & -0.233798 \\
\hline-4.440295 & -3.742105 & -0.722583 \\
\hline-5.352724 & -4.318964 & -0.858212 \\
\hline-3.171733 & -4.320038 & -0.876147 \\
\hline-3.072478 & -5.373766 & -1.135954 \\
\hline-2.042060 & -3.537631 & -0.683581 \\
\hline-1.045601 & -3.963611 & -0.778734 \\
\hline-5.477429 & 1.523272 & 0.851863 \\
\hline-4.281950 & 2.289285 & 1.129996 \\
\hline-4.130138 & 2.675391 & 2.136750 \\
\hline-3.295693 & 2.523007 & 0.149057 \\
\hline-2.415400 & 3.111760 & 0.400971 \\
\hline-3.388616 & 1.891200 & -1.136903 \\
\hline-4.522697 & 1.054267 & -1.366492 \\
\hline-4.583605 & 0.499733 & -2.302599 \\
\hline-5.594846 & 0.937335 & -0.413435 \\
\hline-6.447786 & 0.305784 & -0.652248 \\
\hline-6.487032 & 1.331822 & 1.959506 \\
\hline-5.902696 & 1.287886 & 2.892479 \\
\hline-7.417358 & 2.556405 & 2.029619 \\
\hline-6.846375 & 3.488981 & 2.134604 \\
\hline-8.096358 & 2.472788 & 2.889149 \\
\hline-8.026039 & 2.631724 & 1.116915 \\
\hline-7.275091 & 0.025844 & 1.848817 \\
\hline-7.922437 & 0.012613 & 0.960085 \\
\hline-7.922131 & -0.097513 & 2.727221 \\
\hline-6.592488 & -0.833121 & 1.803311 \\
\hline-2.324595 & 2.071506 & -2.178832 \\
\hline-1.342425 & 2.229178 & -1.716832 \\
\hline-2.547246 & 2.946974 & -2.807373 \\
\hline-2.261711 & 1.190370 & -2.828644 \\
\hline
\end{tabular}

\section{(S-Ru)-8}

$\begin{array}{lrrr}\mathrm{Ru} & -3.350581 & -1.895227 & 0.464603 \\ \mathrm{Cl} & -4.437879 & -0.117773 & -0.734549 \\ \mathrm{O} & 5.287950 & 6.762770 & -0.566295 \\ \mathrm{H} & 5.000231 & 7.420480 & 0.088560 \\ \mathrm{~N} & -2.755420 & -2.609750 & -1.391996 \\ \mathrm{C} & 3.586154 & 3.715439 & -1.984893 \\ \mathrm{H} & 3.970945 & 2.971867 & -2.696364 \\ \mathrm{H} & 2.702349 & 4.176152 & -2.459188 \\ \mathrm{C} & 4.657207 & 4.791024 & -1.764231 \\ \mathrm{H} & 5.589755 & 4.337696 & -1.393407 \\ \mathrm{H} & 4.901509 & 5.281975 & -2.717532 \\ \mathrm{C} & 4.198991 & 5.835668 & -0.757150 \\ \mathrm{H} & 3.330955 & 6.377914 & -1.181140 \\ \mathrm{C} & 3.764380 & 5.178653 & 0.551040 \\ \mathrm{H} & 3.382450 & 5.947089 & 1.243622\end{array}$




\begin{tabular}{|c|c|c|c|}
\hline $\mathrm{H}$ & 4.644598 & 4.724750 & 1.033062 \\
\hline C & 2.672394 & 4.130815 & 0.305535 \\
\hline $\mathrm{H}$ & 1.839057 & 4.652301 & -0.204506 \\
\hline C & 0.919654 & 2.632354 & 1.323059 \\
\hline $\mathrm{H}$ & 0.088044 & 3.225313 & 0.902347 \\
\hline $\mathrm{H}$ & 0.545443 & 2.188337 & 2.257702 \\
\hline C & 1.277865 & 1.523406 & 0.328405 \\
\hline $\mathrm{H}$ & 2.018899 & 0.861350 & 0.806955 \\
\hline C & 1.885190 & 2.119174 & -0.970189 \\
\hline $\mathrm{H}$ & 1.109975 & 2.803131 & -1.369032 \\
\hline C & 3.127613 & 3.017067 & -0.683535 \\
\hline C & 2.130075 & 1.048421 & -2.053753 \\
\hline $\mathrm{H}$ & 2.404439 & 1.541980 & -2.996009 \\
\hline $\mathrm{H}$ & 2.997399 & 0.433412 & -1.773027 \\
\hline C & 0.928260 & 0.121536 & -2.322490 \\
\hline $\mathrm{H}$ & 1.235942 & -0.655057 & -3.039495 \\
\hline $\mathrm{H}$ & 0.111842 & 0.691036 & -2.794693 \\
\hline C & 0.411017 & -0.489440 & -1.014272 \\
\hline C & 0.062099 & 0.699229 & -0.070502 \\
\hline $\mathrm{H}$ & -0.579490 & 1.361999 & -0.681029 \\
\hline C & -0.893921 & 0.096257 & 0.978280 \\
\hline $\mathrm{H}$ & -1.567263 & 0.836781 & 1.430799 \\
\hline $\mathrm{H}$ & -0.352086 & -0.390451 & 1.811105 \\
\hline C & -1.631419 & -0.934560 & 0.154974 \\
\hline C & -0.932039 & -1.206484 & -0.994597 \\
\hline C & 1.461829 & -1.450094 & -0.411952 \\
\hline $\mathrm{H}$ & 1.161402 & -1.778385 & 0.591731 \\
\hline $\mathrm{H}$ & 2.458753 & -0.998406 & -0.336103 \\
\hline $\mathrm{H}$ & 1.546321 & -2.347184 & -1.041564 \\
\hline C & 4.290169 & 2.183899 & -0.113315 \\
\hline $\mathrm{H}$ & 3.998124 & 1.621814 & 0.781715 \\
\hline $\mathrm{H}$ & 5.145118 & 2.813102 & 0.161408 \\
\hline $\mathrm{H}$ & 4.648000 & 1.461072 & -0.858484 \\
\hline C & -1.543734 & -2.148278 & -1.874883 \\
\hline C & -3.498188 & -3.445329 & -2.141945 \\
\hline $\mathrm{H}$ & -4.457344 & -3.737517 & -1.718671 \\
\hline C & -3.075290 & -3.919486 & -3.377455 \\
\hline $\mathrm{H}$ & -3.708044 & -4.605779 & -3.936330 \\
\hline C & -1.832162 & -3.495789 & -3.868678 \\
\hline $\mathrm{H}$ & -1.468133 & -3.852412 & -4.831993 \\
\hline C & -1.074046 & -2.605716 & -3.120859 \\
\hline $\mathrm{H}$ & -0.117070 & -2.242450 & -3.488603 \\
\hline C & -2.570788 & -3.111690 & 2.098189 \\
\hline C & -3.068597 & -1.873323 & 2.625437 \\
\hline $\mathrm{H}$ & -2.392176 & -1.210499 & 3.163440 \\
\hline C & -4.391576 & -1.451216 & 2.365073 \\
\hline $\mathrm{H}$ & -4.711343 & -0.462214 & 2.687456 \\
\hline C & -5.329593 & -2.304105 & 1.669780 \\
\hline C & -4.867452 & -3.528200 & 1.172736 \\
\hline $\mathrm{H}$ & -5.534697 & -4.171148 & 0.600676 \\
\hline C & -3.478522 & -3.877078 & 1.304452 \\
\hline $\mathrm{H}$ & -3.119312 & -4.775905 & 0.806264 \\
\hline C & -1.144418 & -3.541349 & 2.361662 \\
\hline $\mathrm{H}$ & -0.556554 & -2.616874 & 2.479438 \\
\hline C & -1.078169 & -4.317559 & 3.688493 \\
\hline $\mathrm{H}$ & -1.657415 & -5.249770 & 3.618298 \\
\hline $\mathrm{H}$ & -1.486530 & -3.726117 & 4.519151 \\
\hline $\mathrm{H}$ & -0.038900 & -4.578574 & 3.931114 \\
\hline C & -0.530939 & -4.344369 & 1.211632 \\
\hline $\mathrm{H}$ & 0.534041 & -4.526140 & 1.406816 \\
\hline $\mathrm{H}$ & -0.620172 & -3.800931 & 0.263015 \\
\hline $\mathrm{H}$ & -1.015450 & -5.324721 & 1.100605 \\
\hline C & -6.718993 & -1.812111 & 1.407325 \\
\hline $\mathrm{H}$ & -6.674256 & -0.867577 & 0.844680 \\
\hline $\mathrm{H}$ & -7.245669 & -1.617161 & 2.352525 \\
\hline $\mathrm{H}$ & -7.296288 & -2.537827 & 0.823187 \\
\hline C & 2.101452 & 3.560727 & 1.605477 \\
\hline $\mathrm{H}$ & 1.785960 & 4.388947 & 2.259295 \\
\hline $\mathrm{H}$ & 2.888066 & 3.017086 & 2.153596 \\
\hline
\end{tabular}




\section{References}

1. Still, W. C.; Kahn, M.; Mitra, A., Rapid chromatographic technique for preparative separations with moderate resolution. J. Org. Chem. 1978, 43 (14), 2923-2925.

2. Koch, V.; Nieger, M.; Bräse, S., Stille and Suzuki Cross-Coupling Reactions as Versatile Tools for Modifications at C-17 of Steroidal Skeletons - A Comprehensive Study. Adv. Synth. Catal. 2017, 359 (5), 832-840.

3. Mori, H.; Tsuneda, K., Studies on Steroidal Compounds. X. Preparation of $17 \beta$-Chloro Steroids. Chem. Pharm. Bull. 1963, 11 (11), 1413-1417.

4. Comins, D. L.; Mantlo, N. B., Regioselective addition of nucleophiles to 1-(phenoxycarbonyl)-3trialkylstannylpyridinium salts. Tetrahedron Lett. 1987, 28 (7), 759-762.

5. Sheldrick, G., A short history of SHELX. Acta Crystallogr. A 2008, 64 (1), 112-122.

6. Sheldrick, G., Crystal structure refinement with SHELXL. Acta Crystallogr. B 2015, 71 (1), 3-8.

7. Parsons, S.; Flack, H. D.; Wagner, T., Use of intensity quotients and differences in absolute structure refinement. Acta Crystallogr. B 2013, 69 (3), 249-259.

8. Dolomanov, O. V.; Bourhis, L. J.; Gildea, R. J.; Howard, J. A. K.; Puschmann, H., OLEX2: a complete structure solution, refinement and analysis program. J. Appl. Cryst. 2009, 42 (2), 339-341.

9. Weigend, F.; Ahlrichs, R., Balanced basis sets of split valence, triple zeta valence and quadruple zeta valence quality for $\mathrm{H}$ to Rn: Design and assessment of accuracy. Phys. Chem. Chem. Phys. 2005, 7 (18), 3297-3305.

10. Zhao, Y.; Truhlar, D. G., A new local density functional for main-group thermochemistry, transition metal bonding, thermochemical kinetics, and noncovalent interactions. J. Phys. Chem . 2006, 125 (19), 194101.

11. Stephens, P. J.; Devlin, F. J.; Chabalowski, C. F.; Frisch, M. J., Ab Initio Calculation of Vibrational Absorption and Circular Dichroism Spectra Using Density Functional Force Fields. J. Phys. Chem. 1994, 98 (45), 11623-11627.

12. Tao, J.; Perdew, J. P.; Staroverov, V. N.; Scuseria, G. E., Climbing the Density Functional Ladder: Nonempirical Meta--Generalized Gradient Approximation Designed for Molecules and Solids. Phys. Rev. Lett. 2003, 91 (14), 146401.

13. Perdew, J. P., Density-functional approximation for the correlation energy of the inhomogeneous electron gas. Phys. Rev. B 1986, 33 (12), 8822-8824.

14. Becke, A. D., Density-functional exchange-energy approximation with correct asymptotic behavior. Phys. Rev. A 1988, 38 (6), 3098-3100. 